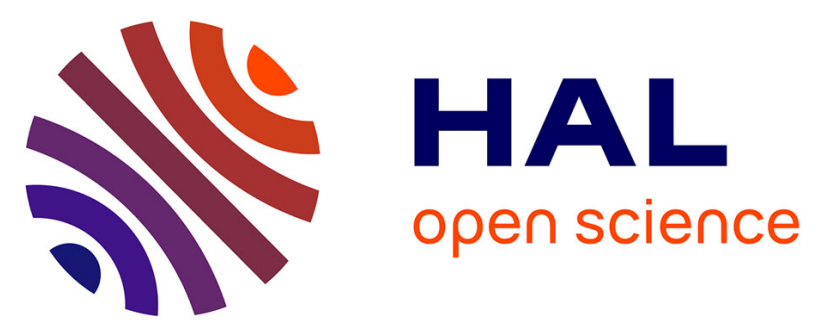

\title{
Microscale prediction of deformation in an austenitic stainless steel under uniaxial loading
}

\author{
Dong-Feng Li, Noel P. O’Dowd, Catrin M. Davies, Shu-Yan Zhang
}

\section{To cite this version:}

Dong-Feng Li, Noel P. O’Dowd, Catrin M. Davies, Shu-Yan Zhang. Microscale prediction of deformation in an austenitic stainless steel under uniaxial loading. European Journal of Mechanics - A/Solids, 2011, 30 (5), pp.748. 10.1016/j.euromechsol.2011.02.002 . hal-00769678

\section{HAL Id: hal-00769678 \\ https://hal.science/hal-00769678}

Submitted on 3 Jan 2013

HAL is a multi-disciplinary open access archive for the deposit and dissemination of scientific research documents, whether they are published or not. The documents may come from teaching and research institutions in France or abroad, or from public or private research centers.
L'archive ouverte pluridisciplinaire HAL, est destinée au dépôt et à la diffusion de documents scientifiques de niveau recherche, publiés ou non, émanant des établissements d'enseignement et de recherche français ou étrangers, des laboratoires publics ou privés. 


\section{Accepted Manuscript}

Title: Microscale prediction of deformation in an austenitic stainless steel under uniaxial loading

Authors: Dong-Feng Li, Noel P. O'Dowd, Catrin M. Davies, Shu-Yan Zhang

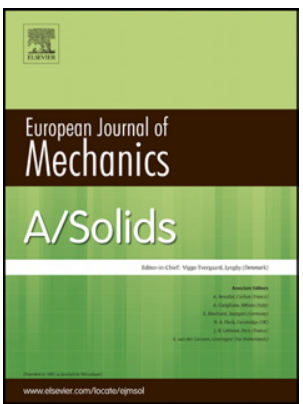

PII:

S0997-7538(11)00020-9

DOI:

10.1016/j.euromechsol.2011.02.002

Reference: EJMSOL 2683

To appear in: European Journal of Mechanics / A Solids

Received Date: 15 November 2010

Revised Date: 10 February 2011

Accepted Date: 11 February 2011

Please cite this article as: Li, D.-F., O'Dowd, N.P., Davies, C.M., Zhang, S.-Y. Microscale prediction of deformation in an austenitic stainless steel under uniaxial loading, European Journal of Mechanics / A Solids (2011), doi: 10.1016/j.euromechsol.2011.02.002

This is a PDF file of an unedited manuscript that has been accepted for publication. As a service to our customers we are providing this early version of the manuscript. The manuscript will undergo copyediting, typesetting, and review of the resulting proof before it is published in its final form. Please note that during the production process errors may be discovered which could affect the content, and all legal disclaimers that apply to the journal pertain. 


\title{
Microscale prediction of deformation in an austenitic stainless steel under uniaxial loading
}

\author{
Dong-Feng Li ${ }^{\mathrm{a}}$, Noel P. O’Dowd ${ }^{*, \mathrm{a}}$, Catrin M. Davies ${ }^{\mathrm{b}}$, Shu-Yan Zhang ${ }^{\mathrm{c}}$ \\ ${ }^{a}$ Department of Mechanical and Aeronautical Engineering, Materials and Surface Science Institute, \\ University of Limerick, Limerick, Ireland \\ ${ }^{b}$ Department of Mechanical Engineering, Imperial College London, South Kensington Campus, London \\ $S W 7$ 2AZ, United Kingdom \\ ${ }^{c}$ ISIS, STFC Rutherford Appleton Laboratory, Chilton Didcot, Oxfordshire OX11 0QX, United Kingdom
}

\begin{abstract}
In this study, the deformation behaviour of polycrystalline austenitic $316 \mathrm{H}$ stainless steel under uniaxial loading is investigated by means of in-situ neutron diffraction (ND) measurement and crystal plasticity-based finite element (FE) modelling. Data have been obtained for the macroscopic stress-strain response and the lattice strain evolution in the longitudinal and transverse direction relative to the uniaxial loading axis. Comparison between the model predictions and the ND measurements suggest that in most cases the FE model can predict the lattice strain evolution at the microscale and capture the general trends observed in the experiments. Both ND measurements and FE modelling simulations identify no micromorphology effect on the longitudinal lattice strain evolution, while the transverse lattice strain response appears to be sensitive to the microstructure, in particular the initial crystallographic orientation of the material.
\end{abstract}

Key words: Austenitic stainless steel; Neutron diffraction; Crystal plasticity; Lattice strain; Finite element;

\section{Introduction}

The macroscopic response of materials is controlled to a large extent by deformation and damage mechanisms operating at the microscale - for polycrystalline engineering alloys the relevant length scale is the grain (crystallite) size. Thus, simulations and experiments conducted at the microscale can provide important insight into the macroscale behaviour of engineering materials or components.

As a non-destructive technique, neutron diffraction (ND) has been employed to explore in-situ the micromechanical deformation behaviour in terms of the evolution of lattice strains for differently oriented grain within a polycrystal under mechanical loading (see, e.g., Clausen

\footnotetext{
*Corresponding author. Phone: +353 (0)61 202545 Fax: +353 (0)61 233766

Email address: noel.odowd@ul.ie (Noel P. O'Dowd)

Preprint submitted to European Journal of Mechanics A/Solids
}

February 10, 2011 
et al., 1998, 1999; Daymond et al., 2000; Pang et al., 2000; Lorentzen et al., 2002; Daymond and Bouchard, 2006; Huang et al., 2008; Marin et al., 2008; Huang et al., 2008). A description of techniques for strain measurement using ND is found in Hutchings et al. (2005). In general, neutron sources may be classed as monochromatic, in which a neutron beam of fixed wavelength is used, or polychromatic, in which a neutron beam with a continuous range of wavelengths is used. For the latter case, the lattice strain is obtained by measuring the time-of-flight (TOF) of diffracted neutrons. For a crystalline material the TOF will depend on the lattice spacing through Bragg's law and the de Broglie relation, (e.g., Hutchings et al., 2005). A polycrystal subjected to a polychromatic neutron beam provides a range of TOFs, as differently orientated grains diffract neutrons of different wavelength (i.e. different speed). As only grain orientations, which satisfy the kinematic condition allow diffraction to occur (see Hutchings et al., 2005), discrete spectra of TOF are obtained from a ND measurement. These TOF spectra can be replotted in terms of lattice spacing, providing a measure of the elastic strain on specific families of lattice planes.

The in-situ lattice strain monitored by diffraction techniques can provide information about microscale heterogeneous stress or strain experienced in polycrystalline aggregates and about the elastic and inelastic anisotropy arising from the crystal structure and other nonlinear physical kinematics, e.g., dislocation slip, phase or microstructure transformation and damaging. Here, the atomic lattice plays the role of an atomic strain gauge to measure the variations of lattice plane spacing represented by the shift of diffraction pattern due to the external loads exposed. Using the time-of-flight in-situ neutron diffraction, the sub-sets of grains (rather than individual grains) are sampled and consequently the mean lattice strains are monitored over a family of grains. The lattice strain evolution in an in-situ test is typically presented against the macroscopic stress applied. When the material deforms plastically, a nonlinear lattice strain response can occur. It is thus expected that polycrystalline models can interpret lattice strain observations and on the other hand such data may be used to calibrate or validate the plasticity models as pointed out by Karato (2009).

Micromechanical modelling techniques have been developed to represent the nonlinear anisotropic deformation at the microscale and the interaction of grains in polycrystalline aggregates. Generally, two approaches have been adopted: self-consistent techniques and finite element (FE) methods. In the elastoplastic self-consistent approach developed by Hill (1965) and extended by Hutchinson (1970), it is assumed that within a polycrystal each grain has an ellipsoidal shape and is embedded in a homogeneous effective medium, such that the solution of Eshelby (1957) can be used to determine the grain response as a function of macroscopic applied loads. The mechanical behaviour of an individual grain is generally represented using the Schmid concept (see, Schmid and Siebel, 1931), with dislocation slip controlled by the shear stress, resolved on an active slip system, comprising close packed planes and slip directions. In the self consistent modelling approach relatively simple strain hardening approximations must be used in order to obtain a closed form solution.

The self-consistent approach treats the intergranular interactions as long-range interactions to maintain the compatibility of the polycrystals. However, grain interactions may also exhibit strong short-range characteristics, such that the individual grain response can be strongly altered by the surrounding grains. To incorporate both long- and short-range 
grain interactions, FE modelling strategies have been developed, where the morphology of the polycrystalline material is represented by a two or three dimensional representative volume element (RVE) with individual grains explicitly modelled essentially within this RVE (see, e.g., Barbe et al., 2001; Delannary et al., 2006; Dunne et al., 2007; Nakamachi et al., 2007). The deformation of individual grains is then typically represented by crystal plasticity theory, based on Schmid's law. In contrast to the self-consistent approach, which assumes a uniform stress or strain distributed within a grain, such micomechanical FE models provide an explicit evaluation of inter-granular (as well as intra-granular) interactions and the type of hardening model used is not restricted to simple models as is the case for self-consistent models. In addition, as finite element models provide an accurate measure of local (microscale) stress/strain distributions, they are also capable of accounting for fracture initiation and intergranular damage evolution due to stress/strain gradients near grain boundaries, which again is not available from self-consistent studies.

To date, self-consistent models have generally been preferred as a technique to predict the lattice strain evolution for comparison with in-situ ND measurements, as FE micromechanical modelling is less computationally efficient, particularly, when modelling a large number of grains within the RVE (e.g., Barbe et al., 2001; Delannary et al., 2006; Dunne et al., 2007; Nakamachi et al., 2007). However, with the development of modern computing technologies (high performance architectures and algorithms), nonlinear FE modelling of a sufficient number of grains has become more practicable. Recently, Wong and Dawson (2010) examined numerically the influence of single crystal elastic anisotropy on the evolution of the lattice strain using a three dimensional crystal plasticity based FE model where the grains of interest are assumed to be virtually rhombic dodecahedral crystals. In their simulations, no comparisons with in-situ ND measurements are presented as their examinations are targeted to virtual materials with different single crystal elastic anisotropy. There are some FE based studies comparing ND measurements with FE predictions for BCC materials (ferritic steels) (see, Dawson et al., 2000; Quinta da Fonseca, 2006). However, these have employed rather simple models of the grain shape (e.g. cubic shape) and low mesh resolution (e.g. a finite single element per grain). To our knowledge this is the first comparison of in-situ ND measurements with high resolution FE models for a FCC material.

The remainder of this paper is laid out as follows: Section 2 describes the experimental procedure (in-situ neutron diffraction). Section 3 outlines the implementation of the FE modelling scheme. In Section 4, the results are presented and the ND measurements and FE modelling predictions are compared. The results are discussed in more detail in Section 5 and concluding remarks are provided in Section 6.

\section{Experimental studies}

\subsection{Material preparation and characterisation}

The material of interest is $316 \mathrm{H}$ austenitic stainless steel, extracted from an ex-service header component supplied by British Energy Group plc, UK. Table 1 gives the chemical composition of the material. Two tensile specimens with diameter $8 \mathrm{~mm}$ and gauge length $25 \mathrm{~mm}$ were machined from the header for the in-situ ND experiments. As the initial texture 
may affect both the macroscopic response and microscale deformation, a texture characterisation was carried out prior to the in-situ ND measurement. Electron backscatter diffraction (EBSD) measurements were carried out at Imperial College London, UK to characterise the morphology of the material. The microstructure and the corresponding crystal orientation distribution shown in Li et al. (2010) indicates a random texture with an average grain size of $45.4 \mu \mathrm{m}$.

\subsection{In-situ neutron diffraction experiment}

The experimental work was conducted on the ENGIN-X instrument at ISIS, UK. For a full description of the ENGIN-X instrument see, e.g., Dann et al. (2004); Santisteban et al. (2006). Figure 1(a) illustrates schematically the arrangement of the in-situ ND measurement at ENGIN-X. The machine operates in polychromatic diffraction mode with a fixed diffraction angle of 45 degrees. The vector difference between the incident and the diffracted beam vectors defines scattering vectors (' $\mathrm{L}$ ' and ' $\mathrm{T}$ ' in Fig. 1(a)), which determine the direction of the measured lattice strain. The use of two detectors for the ND data acquisition at ENGIN-X allows a simultaneous measurement of diffraction patterns relating to longitudinal (L) and transverse (T) scattering vectors, thus providing both longitudinal (parallel to the loading axis) and transverse (perpendicular to the loading axis) lattice strains from a single measurement. The uniaxial tensile test was carried out at room temperature under mixed load and displacement control where machine displacement was controlled to achieve a given strain measured from a standard clip gage extensometer. To investigate the sensitivity to grain morphology, two specimens were measured. The specimens were first loaded within the linear elastic regime to $100 \mathrm{MPa}$ using load control with a total of 10 increments. The specimens were then deformed under displacement control with defined increments (approx. $0.1 \%$ strain) up to a total strain of approx. $10 \%$ strain. The total time taken to complete the in-situ test for a single specimen, allowing time for ND data acquisition for each data point was approx. 21 hours (approx. 9 minutes for each data point). Some relaxation of stress was observed during the ND measurement period (maximum of $10 \%$ in the plastic region). Following the in-situ ND measurements, the EX-SBA software (see, Oliver et al., 2004) was employed to extract the lattice plane spacing data. Figure 1(b) shows a typical diffraction pattern containing a number of diffraction peaks with respect to lattice spacing, $d$. With descending order of $d$-spacing, the Miller indices of lattice planes corresponding to the reflection peaks in Fig. $1(\mathrm{~b})$ are $\{111\},\{200\},\{220\},\{311\},\{222\},\{400\},\{331\}$, $\{420\},\{422\},\{333\}$ and $\{511\}$. All the reflection peaks have unmixed (all even or all odd) Miller indices, which is as expected for an FCC crystal (see, e.g., Hutchings et al., 2005). Furthermore, note that high order reflection peaks ( $\{222\}$ and $\{400\})$ can be identified in the diffraction pattern. The peak with the smallest $d$-spacing corresponds to the two reflection planes $(\{333\}$ and $\{511\})$. Thus, except for this particular peak, each peak in Fig. 1(b) represents a family of grains with a certain crystallographic orientation. In the present work, only the first four peaks, $\{111\},\{200\},\{220\}$ and $\{311\}$ are examined, because of their relatively high peak intensities and low peak-to-peak noises.

Under macroscopic tension, elastic lattice strain occurs as a result of stretching or com- 
pressing of the $\{h k l\}$ lattice plane and the lattice strain is given by

$$
\varepsilon_{h k l}=\frac{d_{h k l}-d_{h k l}^{0}}{d_{h k l}^{0}},
$$

where $\varepsilon_{h k l}$ and $d_{h k l}$ are the elastic lattice strain and the spacing of the $\{h k l\}$ lattice plane, respectively; $d_{h k l}^{0}$ is the reference (unstretched) $\{h k l\}$ lattice spacing. Ideally, $d_{h k l}^{0}$ should be measured from an unstrained cubic crystal. However, internal stress resulting from the fabrication/maching process and strain history cannot generally be avoided, so that at grain level there may be non-zero lattice strains even under macroscopic stress free conditions. An alternative approach adopted here is to use a reference value of $d_{h k l}^{0}$ measured from a load-free specimen. Thus, all strains are measured relative to this initial point. Typically a minimum stress $(5-10 \mathrm{MPa})$ is required to hold the specimen in place for ND data acquisition, so it is not trivial to measure directly the load-free $d_{h k l}^{0}$. Some researchers use the lattice plane spacing measured at the minimum load as an approximation of the loadfree $d_{h k l}^{0}$ (see, e.g., Clausen et al., 1999; Quinta da Fonseca, 2006). In the present study, the load-free $d_{h k l}^{0}$ was estimated from a linear least-squares fit to the measured data with applied stress less than $100 \mathrm{MPa}$, as in Pang et al. (2000).

As shown in Fig. 1(a), the diffraction peak information recorded by the right and left detectors reflect the lattice plane stretches along longitudinal and transverse scattering vectors, respectively. Thus, lattice strain responses in the longitudinal and transverse directions can be obtained directly from Eqn. 1 during unaxial tensile loading. In the current work, the neutron irradiation gauge volume $(7 \mathrm{~mm} \times 4 \mathrm{~mm} \times 4 \mathrm{~mm})$ is located at the center of the specimen. Note that the average grain size of the as-received material is $45.4 \mu \mathrm{m}$. Thus, a large number of grains (approx. one million) can be monitored by ND using the above gauge volume. As mentioned earlier, each reflection peak of interest corresponds to a specific family of grains and incorporates the contributions from all grains within this grain family in the irradiation gauge volume, such that the measured lattice strain reflects the 'average' lattice plane stretches of the specific grain populations.

\section{Finite element modelling}

\subsection{Constitutive law and material properties of single crystal}

Inelastic deformation within grains is assumed to occur due to dislocation slip according to the Schmid concept (Schmid and Siebel, 1931), with deformation on individual slip systems controlled by the resolved shear stress on that system.

The finite-element (FE) framework of crystal plasticity developed by Meissonnier et al. (2001) is employed to represent the response of FCC stainless steel crystals with twelve octahedral slip systems, $\langle 110\rangle\{111\}$, indicating the family of slip directions and slip plane, respectively. The constitutive law associated with stress rate, $\dot{\boldsymbol{\sigma}}$ and strain rate, $\dot{\boldsymbol{\varepsilon}}$ is given by

$$
\dot{\boldsymbol{\sigma}}=\mathbb{C}\left(\dot{\varepsilon}-\dot{\varepsilon}^{p}\right)
$$


where $\mathbb{C}$ and $\dot{\boldsymbol{\varepsilon}}^{p}$ indicate the elastic stiffness tensor and inelastic strain rate, respectively and $\otimes$ denotes the tensor product. Based on the kinematics of slip motion in Asaro and Rice (1977), the time rate of inelastic strain is given by

$$
\dot{\varepsilon}^{p}=\frac{1}{2} \sum_{\alpha=1}^{12} \dot{\gamma}^{\alpha}\left(\boldsymbol{m}^{\alpha} \otimes \boldsymbol{n}^{\alpha}+\boldsymbol{n}^{\alpha} \otimes \boldsymbol{m}^{\alpha}\right),
$$

where $\dot{\gamma}^{\alpha}, \boldsymbol{m}^{\alpha}$ and $\boldsymbol{n}^{\alpha}$ are the slip rate, the slip direction and the normal direction to the slip plane of the slip system $\alpha$, respectively. The flow rule to determine the slip rate is give by

$$
\dot{\gamma}^{\alpha}=\dot{\gamma}_{0} \exp \left\{-Q\left\langle 1-\left\langle\left|\widetilde{\tau}^{\alpha}\right|-\widetilde{S}^{\alpha}\right\rangle^{p}\right\rangle^{q}\right\} \operatorname{sgn}\left(\widetilde{\tau}^{\alpha}\right), \alpha=1, \ldots, 12,
$$

where $Q$ is a material constant; $p, q$ and $\dot{\gamma}_{0}$ are the exponents and pre-exponential constants; the brackets $\langle$. $\rangle$ imply that $\langle x\rangle \equiv x$ for $x>0$ and $\langle x\rangle \equiv 0$ for $x \leq 0$. In Eqn. (4), $\widetilde{S}^{\alpha}$ is the dimensionless slip resistance and $\widetilde{\tau}^{\alpha}$, the dimensionless resolved shear stress, which is given by

$$
\widetilde{\tau}^{\alpha}=\frac{\boldsymbol{\sigma}:\left(\boldsymbol{m}^{\alpha} \otimes \boldsymbol{n}^{\alpha}\right)}{\hat{\tau}_{0} \mu / \mu_{0}},
$$

with $\hat{\tau}_{0}$ the lattice friction stress at $0 \mathrm{~K}$ and $\mu$ and $\mu_{0}$ the shear modulus at the current temperature and $0 \mathrm{~K}$, respectively and : denotes the double contraction. Note that in Eqn. 4 only one internal variable, $\widetilde{S}^{\alpha}$ is used to account for the slip resistance (strain hardening), which differs from the approach in Meissonnier et al. (2001), where both isotropic and kinematic variables were used. As monotonic loading is examined here, a single internal variable is sufficient to represent the strain hardening response of a slip system. Future studies will examine cyclic response of the material. The evolution of $\widetilde{S}^{\alpha}$ is given by

$$
\dot{\widetilde{S}}^{\alpha}=\widetilde{h}_{s}\left(\widetilde{S}_{s a t}-\widetilde{S}^{\alpha}\right)\left|\dot{\gamma}^{\alpha}\right|, \alpha=1, \ldots, 12
$$

where the dimensionless static recovery coefficient, $\widetilde{h}_{s}$ and the dimensionless saturation slip resistance, $\widetilde{S}_{\text {sat }}$ are defined as

$$
\begin{aligned}
\widetilde{h}_{s} & =\frac{h_{s}}{S_{\text {sat }}-S_{0}}, \\
\widetilde{S}_{\text {sat }} & =\frac{S_{\text {sat }}}{\hat{\tau}_{0}},
\end{aligned}
$$

with $S_{0}$ and $S_{\text {sat }}$ the initial and saturation values of slip resistance.

The material of interest, $316 \mathrm{H}$ stainless steel, is a polycrystalline FCC austenitic FeCr-Ni alloy. Its single crystalline counterpart has shown significant elastic anisotropy (see Ledbetter, 1981). The three independent elastic constants, $C_{11}, C_{22}, C_{44}$ for $316 \mathrm{H}$ were obtained by Daymond and Bouchard (2006) from ND measurements and are adopted in the current study as shown in Table 2 . The shear modulus, $\mu_{0}$ at $0 \mathrm{~K}$ needed for the flow rule (Eqn. 4), was obtained by extrapolating the modulus data of Daymond and Bouchard (2006) 
and is also given in the table. The fitted flow rule and slip resistance evolution (isotropic hardening) parameters, used to predict the post yield tensile response of the polycrystalline material at room temperature, are given in Table 3. A total of 8 flow rule parameters in addition to $\mu_{0}$ are required to fully describe the material response. We calibrate the eight flow parameters by comparison with the monotonic tensile curve, as experimental data of the single crystalline austenitic stainless steel are rare. Additional validation of the model is obtained from the neutron diffraction data to be discussed in section 4.

\subsection{Representative volume element construction and discretisation}

For sufficient accuracy, the FE model requires an accurate representation of the material micro-morphology, which may be obtained from experimental techniques, e.g., X-ray tomography, electron back-scatter diffraction (EBSD), or focused ion beam (FIB) serial sectioning. However, it is difficult for these experimental techniques to acquire both grain topologies and crystallographic orientation of grains particularly of a large number (i.e., several thousands) of grains. To date, Voronoi tessellation techniques have been used to approximate the micromorphology of polycrystals due to its efficiency in particular for aggregates with large number of grains (see, e.g., Barbe et al., 2001; Fritzen et al., 2009). From a modelling point of view, there is relatively little information in the literature regarding the effect of grain shape and crystallographic orientation on lattice strain response, although grain boundary morphology may significantly influence the deformation response at the microscale by introducing considerable local gradients of stress and strain near grain boundaries.

In the present work, two RVE configurations, one composed of 1088 identical, hexagonal grains and the other with grains of random shape and size, are used to represent a polycrystal of $316 \mathrm{H}$ stainless steel. Preliminary results for the hexagonal model were presented in $\mathrm{Li}$ et al. (2010). Figure 2(a) shows the RVE containing 1200 randomly shaped grains. For a mean grain size of $45.4 \mu \mathrm{m}$, the RVE has a dimension of $1.57 \mathrm{~mm} \times 1.57 \mathrm{~mm}$ (two grains are labelled G1 and G2 in Fig. 2(a) and results from these particular grains are presented for illustrative purposes in Section 4). Voronoi tessellation has been employed to construct the RVE. The centroidal Voronoi tessellation algorithm (see, Du et al., 1999) has been adopted to avoid grain shape deviation which may result from the use of the standard Voronoi algorithm (see, e.g., Gervois et al., 1992; Fritzen et al., 2009).

Mesh generation of the RVE is performed using the software, GMSH (see, Geuzaine and Remacle, 2009). In the present work, the RVE has approx. 70,000 generalised plane strain elements and ABAQUS (2009) is used to carry out the nonlinear FE modelling analysis in a high-performance computing environment. Figure 2(b) shows the typical finite element mesh at the right corner of RVE (see, Fig. 2a). The dark solid lines represent grain boundaries. Typically 60 linear four-node quadrilateral elements per grain are used in the simulations.

To be consistent with the random texture characterised by EBSD measurement in Li et al. (2010), the RVE grains are assigned with uniformly randomised crystallographic orientations in terms of three Euler angles. Figure 3 shows the inverse pole figure of the FE model in the loading direction. To examine the effect of crystallographic orientations, in addition to the orientation map of Fig. 3, two further orientation maps have been randomly generated. 


\subsection{Boundary conditions and post-processing}

To simulate the macro- and micro-scopic deformations of the polycrystal, the RVE is subjected to tensile loading along the $x_{2}$ direction (See, Fig. 2a) under load control. Periodic boundary conditions are applied to the RVE to constrain the edges for spatial periodicity of deformation, which also requires that corresponding RVE edges have identical discrete nodal arrangements. No special interface algorithms are employed to represent the grain boundary response during deformation - the interfaces between grains are assumed to be perfect mechanical bonds.

The lattice strain values extracted from the ND measurements represent an average measure of the elastic strain over all grains within the irradiated gauge volume with suitable crystallographic orientations. Therefore, the elastic strain results from the corresponding grains in the FE model are also averaged for comparison with the ND results, allowing for a 4 degree tolerance for the plane normal. In other words, the elastic strains of grains orientated within 4 degrees to the relevant reflection position are averaged. The chosen grain members are indicated on the inverse pole map of Fig. 3 with a ' + ' sign for the four reflection planes, $\{200\},\{220\},\{111\}$ and $\{311\}$. Note that for the random orientation shown in the figure, typically only 10 grains contribute to each lattice plane result.

The current study is limited to a small strain analysis and does not account for grain geometry changes. Thus, the coupling between local texture evolution and deformation in terms of lattice rotation within crystals to form grain substructures is not taken into account. In addition, our study considers two-dimensional grains (see Fig. 2) ignoring threedimensional effects. Extension to three-dimensional grain geometries and finite strain kinematics will be considered in further work (see, Li and O'Dowd, 2011).

\section{Results}

\subsection{Macroscopic mechanical response}

Figure 4 shows macroscopic engineering stress-strain curves obtained from the in-situ ND experiment using the force from the machine load cell and the extensometer strain data. Each experimental data point represents the average stress/strain recorded during the ND acquisition period (9 minutes per data point) such that the uncertainties of measurements arise from the deviations during averaging. The current work examined two specimens and no significant difference in the tensile response was observed for the two specimens. Therefore, the tensile data for only one specimen are given here.

The lines in Fig. 4 represent the result from the FE model. There are six sets of FE results in the figure, though individual lines are difficult to distinguish. Three solid lines represent the results of the RVE made up of polygonal grains for three distinct random orientation distributions. The three dash lines indicate the results from the regular RVE (hexagonal grains) with three random orientation distributions. The model parameters in Eqns. 4 and 6 have been chosen to provide the best fit over the deformation range for both RVE descriptions. (Note that no fitting was required to match the linear elastic response and the overall modulus obtained from the FE analysis is within $2 \%$ of the measured macroscopic 
value.) The numerical predictions show good consistency at the macroscopic scale regarding the influence of grain shapes and crystallographic orientation distributions.

\subsection{In-situ lattice strain response during tensile deformation}

\subsubsection{Results from ND measurements}

Figure 5 provides the response from the ND measurements for the $\{200\}$ and $\{220\}$ lattice planes. These planes experience the largest and smallest lattice (elastic) strain levels, respectively, in the longitudinal direction (these planes also exhibit the most nonlinearity in the response). The data shown in the figure are from the two specimens measured in this study (triangles and circles). Also included in Fig. 5(a) and (c) are the data of Daymond and Bouchard (2006) for a similar material (squares). For clarity, error bars are not included for the data of Daymond and Bouchard (2006) - their magnitude is similar to that of the current measurement. The data in Daymond and Bouchard (2006) extends only to a maximum lattice strain level of approx. 2,500 microstrains (corresponding to approx. $3 \%$ global strain). In the current study, lattice strains of up to approx. 4,000 microstrains have been considered (corresponding to approx. $10 \%$ global strain) to provide a more complete description of the plastic response of the polycrystal. No data were reported in Daymond and Bouchard (2006) for the $\{220\}$ reflection.

In Fig. 5, the applied stress (obtained from the applied load and representing an average over all grains in the polycrystal) is plotted against the local elastic (lattice) strain (an average over all reflecting grains of the relevant grain family). The experimental lattice strains are obtained from Eqn. 1 and the experimental uncertainties arise from the standard deviations determined by the Rietveld fit (see, Rietveld, 1969) to data of the type shown in Fig.1(b).

The present data are in good agreement with that of Daymond and Bouchard (2006) the small variations observed may be due to differences in the material between the two studies (the data of Daymond and Bouchard (2006) are from a different batch of $316 \mathrm{H}$ material). Figures 5(a) and (b) also show a good consistency in the measurements between the two specimens which suggests no microstructural effects on the longitudinal lattice strain response, for a typical randomly textured material. However, some variation is noted in Fig. 5(c), indicates some variability between specimens for the transverse strain, with the data of Daymond and Bouchard (2006) closest to the results from specimen 2. This issue will be discussed in more detail in the Discussion section.

The lattice strain response in Fig. 5 can be partitioned into a linear regime with applied stress below approx. $200 \mathrm{MPa}$ and a nonlinear regime with applied stress exceeding $200 \mathrm{MPa}$. If local stress against local elastic strain were plotted, a linear plot would be produced over the full extent of strain (see Eqn. 2). In the linear elastic regime, it can also be shown that the local stress is linearly related to the global (applied) stress. As the applied stress is increased, however, inelastic strain is generated within the polycrystal leading to a nonlinear relationship between global stress and global strain and thus a nonlinear relationship between global stress and local strain. For a particular grain family, the magnitude of nonlinearity represented by the deviation from the linear extrapolation of the elastic regime will depend on the grain family orientation (through the Schmid factors for the individual slip systems 
within the grain family), the inelastic constitutive response (strain hardening) and, to a lesser extent, interactions with neighbouring grains. Such measured trends can provide useful validation data for comparison with an FE model, as discussed in the next section.

\subsubsection{Comparison between ND measurements and FE predictions}

Figures 6 and 7 provide a comparison between the ND measurements and FE predictions for stress vs. lattice strain for the four strongest reflections. Figure 6 provides the results for the longitudinal strain and Fig. 7 the result for the transverse strain. The results of six sets of $\mathrm{FE}$ analyses are shown in each figure, corresponding to three random lattice orientations and two RVE geometries.

The predictions of longitudinal lattice strain from the FE analysis, shown by the solid and dash lines in Fig. 6 are seen to be in excellent agreement with the ND predictions in the linear regime. Table 4 provides a direct comparison of the measured lattice plane moduli (representing the linear relationship between applied stress and lattice strain) and the FE predictions. The uncertainties of the measurements arise from the variability between the two specimens measured. The FE model's uncertainties arise from the deviation between the six sets of simulations. (The square brackets for the $\{220\}$ and $\{311\}$ transverse moduli data indicate the range of all six sets of modelling results and will be discussed later in the paper.) Also included in Table 4 are the lattice plane moduli for the case of a single crystal under uniaxial loading along the relevant local crystallographic orientation. (Details of the calculation procedure is given in the appendix). The difference between the single crystal longitudinal moduli and the polycrystalline results, provides a measure of the elastic grain interactions, i.e. the $\{200\}$ grain family longitudinal modulus is increased, while the modulus for the $\{111\}$ grain family is decreased, relative to the single crystal value.

In the nonlinear regime (applied stress $>200 \mathrm{MPa}$ ), the longitudinal ND results in Fig. 6 show that the $\{200\}$ and $\{220\}$ grain families exhibit a strong nonlinearity compared to the $\{111\}$ and $\{311\}$ grain families. These results are consistent with that reported by Clausen et al. (1998). In these figures, the FE model captures the major trends in the ND measurement data for the longitudinal strain, e.g. largest lattice strains experienced by the $\{200\}$ family, lower strains experienced by the $\{111\}$ and $\{220\}$ families and a reasonably good estimate of the strain levels are provided by the model. The small amount of variation in the six sets of modelling results for each grain family indicates a relatively weak effect of micro-morphology on the average lattice strain responses, consistent with the experimental observations (low variability in measured longitudinal lattice strain between specimen 1 and specimen 2).

Figure 7 provides the transverse response from the ND measurements for the four lattice planes examined. Under uniaxial conditions the transverse strain is negative (Poisson effect). The lines labelled A, B and C correspond to the predictions from the three distinct random orientation distributions generated within the RVE, and the solid and dash lines correspond to the polygonal and hexagonal grain shapes, respectively.

It may be seen in Fig. 7 that the model follows the trends of the data, for example the decrease in magnitude of the transverse lattice strain for the $\{200\}$ grain family with increasing applied stress near $200 \mathrm{MPa}$, (see Fig. 7a). It may also be noted in Fig. 7(a) that 
in the nonlinear regime there is a measurable difference between the transverse response of the two specimens for the $\{200\}$ reflection. This trend is also seen in the FE model predictions, where the results from the three random orientation distributions $(\mathrm{A}, \mathrm{B}$ and $\mathrm{C}$ ) are considerably different. Good agreement is obtained between the model and measurement for orientation $\mathrm{B}$ and $\mathrm{C}$, but poor agreement for orientation $\mathrm{A}$. This suggests that the transverse lattice response for the $\{200\}$ reflection is sensitive to the microstructure of asreceived material, particularly when the deformation is in the inelastic regime. Note that grain shape (i.e. polygonal vs. hexagonal, solid line vs. dash line, respectively) has a weak effect on the predicted strains compared to the difference between the different 'random' orientation distributions. Some variability between the two specimens is also seen for the $\{220\}$ reflection, with orientations $\mathrm{A}$ and $\mathrm{C}$ giving the best agreement for the $\{220\}$ reflection. For the $\{111\}$ and $\{311\}$ reflections, good agreements can be observed in Figs. $7(\mathrm{c})$ and (d), between the experiment and the model with the deformation essentially remaining within the linear regime, with some variation observed for the two specimens in the case of the $\{111\}$ reflection in Fig. $7(\mathrm{c})$.

The measured and predicted transverse lattice plane moduli are given in Table 4. It can be seen that for the $\{200\},\{111\}$ and $\{311\}$ reflections the predictions are in reasonable agreement with the corresponding measurements. The theoretical single crystal calculations are also presented in Table 4 (the detailed calculation procedures are described in the appendix). Note that the $\{220\}$ and $\{311\}$ transverse moduli have multiple values, since the transverse lattice plane modulus depends on the Miller indices of both the reflection and the local (crystallographic) loading direction. This is consistent with the fact that a large variability was obtained for the transverse modulus from the measurement and the FE model. The single crystal analysis allows for a positive transverse modulus for a $\{220\}$ oriented crystal (see Table 4). Although no positive transverse moduli were measured or predicted for the $\{220\}$ grain family, individual grains in the FE model did demonstrate positive transverse moduli (the model results are an average over all reflecting grains for a particular grain family). The results for the $\{111\}$ grain family are also shown in the table, with the FE model predicting a considerable variation in transverse modulus for the 6 simulations ( $>10 \%$ variation), while both the experiment data and single crystal analyses indicate a low variability for this orientation.

\subsection{Predicted Inter- and intra-granular stresses within polycrystals}

As discussed in Section 2, the in-situ ND measurements record the average lattice strain response of a grain family as a function of applied stress. In reality, each individual grain in a family may experience a different response due to the interaction with its individually distinct grain neighbourhood. Figure 8 provides the stress-strain response averaged over each individual grain for the four longitudinal grain families of interest from the FE analysis (in these figures orientation ' $\mathrm{A}$ ' has been chosen to illustrate the trends - similar results are seen for the other orientations). The labels G1 and G2 on Fig. 8(b) represent the results for two grains identified in Fig. 2(a), which correspond to the lowest and highest stress, respectively for the $\{220\}$ grain family. For each grain family, the variations among individual grains arise mainly from the inter-granular stresses introduced during deformation, in particular plastic 
deformation. The modulus of the grain family representing the linear relationship between the local stress and strain of grain individuals is also given in the figure (note that the scatter in this figure represents the variation in modulus between individual grains, the scatter in Table 4 represents the variation between simulations of different overall micro-morphology). Figure 8 ( shows that in the nonlinear region the $\{200\}$ grain family generally sustains the lowest level of tensile stress, while the $\{220\}$ and $\{111\}$ grain families experience the highest level of tensile stress. Moreover, strong local strain hardening behaviour can be identified for the $\{220\}$ and $\{111\}$ grain families. The scatter observed in the results suggests that strong short-range grain interaction may occur in polycrystals under plastic deformation.

Figure 9 plots the stress contours of the longitudinal $\{200\}$ grains showing the nonuniform stress distribution within a grain i.e. intra-granular stress (results are for a $400 \mathrm{MPa}$ macroscopic applied stress). Here, two representative grains with low and high local stresses (G1 and G2 as shown in Fig. 2a, respectively) are shown. The extreme values of tensile stress (the lowest and highest) tend to occur at grain boundaries and the high stress gradients take place near the grain boundaries.

\section{Discussion and interpretations of the results}

To examine the lattice strain evolution, the present investigation used an RVE-based FE modelling approach, in conjunction with ND measurements. The proposed FE modelling scheme takes the short-range interaction between grains into account and allows for realistic microstructural morphologies and nonuniform deformation within grains. The overall predictions from the modelling study show reasonable agreement with the ND measurements.

\subsection{Effect of microstructural morphology on observed response}

In this study we examined two nominally identical specimens with random crystal orientation using ND. As expected, there was no observable influence of micromorphology on the macroscopic response for the two specimens. At the microscale, the longitudinal lattice strain response was almost identical for the two specimens, but the transverse lattice strain response, particularly for the $\{200\}$ and $\{111\}$ reflections, appears to be sensitive to material microstructure. In the numerical analysis, six sets of modelling results were examined (varying grain shape and orientation distributions). Again, at the macroscopic scale the stress-strain response was not sensitive to changes in the grain shape and orientation. In terms of lattice strain evolution at the microscale, the modelling predictions are insensitive to local microstructure, except for the transverse strain for the $\{200\}$ grain family (with significant deviations only in plastic region) and the $\{220\}$ grain family. The strongest effect seen is from orientation rather than grain shape. The identified sensitivity to the micromorphology can be explained as follows: As shown by the predictions for a single crystal material

in Table 4, the transverse elastic lattice response depends strongly on the crystallographic loading direction, particularly for the transverse $\{220\}$ lattice plane (the transverse lattice plane modulus can be negative or positive and can vary widely, depending on the local orientation with respect to the loading axis). Therefore the average transverse response obtained 
from an FE simulation with a finite number of grains may exhibit significant scatter, (Table 4). It is expected that as the number of grains in the FE model is increased, approaching a true random grain distribution, the predicted variation would become weaker. However, we note that for the experimental results, with a large gauge volume and thus large number of grains (approx. one million grains) the uncertainties in the modulus for the transverse lattice planes are measurably greater than for the longitudinal lattice planes (Table 4).

\subsection{Comparison between modelling and ND results}

The general trends of the measured nonlinearity in the longitudinal $\{200\}$ grain family can be captured by the current crystal plasticity model, as shown in Fig 6(a). However, the nonlinear response of the longitudinal $\{220\}$ grain family is not well predicted, as shown by Fig 6(b). The reasons for this are believed to be multifold.

Firstly, in the current FE model the RVE is assumed to be free of initial residual stress/strain at the grain level. In reality, internal stress/strain introduced by the deformation history cannot be avoided. It is likely that these initial internal stress/strain, while having negligible effect at the macroscale, will affect the subsequent lattice strain evolution during uniaxial loading. Further modelling and experimental work is required to examine this issue.

Secondly, in the current work, the crystal plasticity formulation is employed based on a small strain assumption. In reality, however, when tensile elongation is sufficiently large, lattice reorientation may occur due to grain interactions at the grain boundaries and plastic anisotropy. Therefore, mechanisms associated with finite strain formulation may introduce considerable effects on the microscale response. For example, during tensile deformation, grains in the $\{220\}$ grain family tends to reorient towards the $\{111\}$ or $\{200\}$ orientation, introducing texture into the specimen even for an initially texture-random specimen. This trend is illustrated in Fig. 10, which shows the changes in peak intensity during deformation. Note that the peak intensity for the $\{220\}$ grain family decreases by about $30 \%$ while the peak intensity for the $\{111\}$ or $\{200\}$ grain families increases, (similar trends are seen in Pang et al. (2000)). Thus, the texture development may affect the lattice strain evolution at large strains. Work is ongoing to take these effects into account (see, Li and O'Dowd, 2011).

Thirdly, as pointed out by Clausen et al. (1999) and Pang et al. (2000), inelastic deformation within austenitic steel may involve not only dislocation slip-deformation twinning may also occur due to the low stacking fault energy. The present FE model does not incorporate deformation twinning, though in principle such a mechanism can be introduced (see, e.g., Kalidindi, 1999; Choi et al., 2010).

Finally, the results presented assume a $2 D$ crystal geometry under generalised plane strain conditions. Inaccuracies in the prediction may be introduced by the assumption of a $2 D$ deformation state as it assumes a uniform distribution of stress and strain along the normal direction and zero out-of-plane shear stress and strain components. When material deforms elastically, near linear relationships between lattice strain and macroscopic stress are measured and well predicted by the present model. However, when plastic flow dominates, considerable nonlinear response develops for some reflections due to crystallographic slip which 
is not precisely captured by the current $2 D$ models. It is expected that the agreement may be improved through the use of a full $3 D$ representation of the local strain state. This is being examined in the ongoing work (see, Li and O'Dowd, 2011).

\section{Conclusions}

Deformation at the macroscopic and microscopic scale for $316 \mathrm{H}$ stainless steel under uniaxial loading has been examined using FE modelling and in-situ neutron diffraction. Strongly nonlinear lattice strain response under uniaxial loading has been identified for $\{200\}$ and $\{220\}$ grains in the longitudinal direction and transverse $\{200\}$ grains in the transverse direction. These trends have been seen in the ND investigation and the FE model. The ND measurements and FE model confirm that no micromorphology effects are identified for the macroscopic stress-strain response and the longitudinal lattice strain response. The ND measurement reports a micromorphology effect on the transverse $\{200\}$ and $\{111\}$ reflections, particulary in the nonlinear region. The FE model predicts a stronger micromorphology effect on the transverse lattice strain response, in particular for the $\{220\}$ and $\{200\}$ grain families. It is expected that with increasing the number of grains in the FE model, approaching a true random grain distribution, the micromorphology effect of transverse lattice strain would become weaker.

\section{Acknowledgement}

The support of Science Foundation Ireland (SFI) under grant 08/RFP/ENM1477 and the Irish Center for High-End Computing (ICHEC) is acknowledged. Some computational work was carried out using the MSSI AMPS cluster, enabled under the framework of the INSPIRE programme, funded by the Irish Government's Programme for Research in Third Level Institutions, Cycle 4, National Development Plan 2007-2013. We are also grateful to British Energy Group plc, UK, for the supply of material. John Burkardt is acknowledged for providing the Voronoi tessellation code from the webpage: http://people.sc.fsu.edu/ burkardt/cpp_src/cvt/cvt.html.

\section{References}

ABAQUS V6.9, 2009. Hibbitt, Karlsson and Sorensen Inc., Providence, RI, USA.

Asaro, R.J., Rice, J.R., 1977. Strain localization in ductile single crystals. J. Mech. Phys. Solids, 25, $309-338$.

Barbe, F., Decker, L., Jeulin, D., Cailletaud, G., 2001. Intergranular and intragranular behaviour of polycrystalline aggregrates Part. I: F.E. Model. Int. J. Plast. 17,513-536.

Choi, S.H., Kim, D.H., Park, S.S., You, B.S., 2010. Simulation of stress concentration in Mg alloys using the crystal plasticity finite element method. ACTA Mater., 58, 320-329.

Clausen, B., Lorentzen, T., Bourke, M.A.M., Daymond, M.R., 1999. Lattice strain evolution during uniaxial tensile loading of stainless steel. Materials Science and Engineering A, A259, 17-24.

Clausen, B., Lorentzen, T., Leffers, T., 1998. Self-consistent modelling of the plastic deformation of F.C.C. polycrystals and its implications for diffraction measurements of internal stresses. ACTA Mater., 46(9), 3087-3098. 
Dann, J.A., Daymond, M.R., James, J.A., Santisteban, J.R., Edwards, L., 2004. A comparison between Engin and ENGIN-X, a new diffractometer optimized for stress measurement. Physica B: Condensed Matter 350 (1-3), e511-e514.

Dawson, P., Boyce, D., MacEwen, S., Rogge, R., 2000. Residual strains in HY100 polycrystals: Comparisons of experiments and simulations. Metallurgical and Materials Transactions A, 31A, 1543-1555.

Daymond, M.R., Bouchard, P.J., 2006. Elastoplastic deformation of 316 stainless steel under tensile loading at elevated temperatures. Metallurgical and Materials Transactions A, 37A, 1863-1873.

Daymond, M.R., Tomé, C.N., Bourke, M.A.M., 2000. Measured and predicted intergranular strains in textured austenitic steel. ACTA Mater., 48, 553-564.

Delannay, L., Jacques, P.J., 2006. Kalidindi SR. Finite element modeling of crystal plasticity with grains shaped as truncated octahedrons. Int J Plasticity, 22(10), 1879-98.

Du, Q., Faber, V., Gunzburger, M., 1999. Centroidal Voronoi Tessellations: Applications and Algorithms, SIAM Review, 41, 637-676.

Dunne, F.P.E., Rugg, D., Walker A., 2007. Lengthscale-dependent, elastically anisotropic, physically-based hcp crystal plasticity: Application to cold-dwell fatigue in Ti alloys. Int J Plasticity, 23(6), 1061-83.

Eshelby, J.D., 1957. The determination of the elastic field of an in ellipsoidal inclusion, and related problems. Proc. Roy. Soc. Lond. A, 241, 376-396.

Fritzen, F., Böhlke, T., Schnack, E., 2009. Periodic three-dimensional mesh generation for crystalline aggregates based on Voronoi tessellations. Comput. Mech., 43, 701-713.

Gervois, A., Troadec, J., Lemaitre, J., 1992. Universal properties of Voronoi tessellations of hard discs. J. Phys. A 25,6169-6177.

Geuzaine, C., Remacle, J.F., 2009. Gmsh: A 3-D finite element mesh generator with built-in pre- and post-processing facilities. Int. J. Numer. Meth Eng., 79(11), 1309-1331.

Hill, R., 1965. Continuum micro-mechanics of elastoplastic polycrystals. J. Mech. Phys. Solids, 13(2), 89-101

Huang, E.W.,Barabash, R., Jia, N., Wang, Y.D., Ice, G.E., Clausen, B., Horton, J., Liaw, P.K., 2008. Slip-System-Related Dislocation Study from In-Situ Neutron Measurements. Metallurgical and Materials Transactions A. 39A(13), 3079-3088.

Huang, E.W.,Barabash, R., Wang, Y.D., Clausen, B., Li, L., Liaw, P.K., Ice, G.E., Ren, Y.,Choo, H., Pike, L.M., Klarstrom, D.L., 2008. Plastic behavior of a nickel-based alloy under monotonic-tension and low-cycle-fatigue loading. Int. J. Plast., 24, 1440-1456.

Hutchings, M.T., Withers, P.J., Holden, T.M., Lorentzen, T., 2005. Introduction to the Characterisation of Residual Stresses by Neutron Diffraction. London: CRC Press, Taylor \& Francis.

Hutchinson, J.W., 1970. Elastic-Plastic behaviour of polycrystalline metals and composites. Proc. Roy. Soc. Lond. A, 319, 247-272.

Kalidindi, S.R., 1999. Incorporation of deformation twinning in crystal plasticity model. J. Mech. Phys. Solids, 46, 267-290.

Karato, S.I., 2009. Theory of lattice strain in a material undergoing plastic deformation: Basic formulation and applications to a cubic crystal. Physical Review B, 79, 214106.

Ledbetter, H.M., 1981. Predicted single-crystal elastic constants of stainless-steel 316. British Journal of Non-Destructive Testing, 23, 286-287.

Li, D.F., O'Dowd, N.P., Davies, C.M., Zhang, S.Y., 2010. Evaluating the mechanical behaviour of 316 stainless steel at the microscale using finite element modelling and in-situ neutron scattering. Proceedings of 2010 ASME Pressure Vessels and Piping Division Conference - ASME PVP/K-PVP 2010 Conference, Jul. 18th-2nd 2010, Bellevue, WT, USA.

Li, D.F., O'Dowd, N.P., 2011. Investigation of finite deformation and texture evolution in austenitic stainless steels under uniaxial tensile loading. Manuscript in preparation.

Lorentzen, T., Daymond, M.R., Clausen, B., Tomé, C.N., 2002. Lattice strain evolution during cyclic loading of stainless steel. ACTA Mater., 50, 1627-1638.

Marin, T., Dawson, P.R., Gharghouri, M.A., Rogge, R.B., 2008. Diffraction measurements of elastic strains in stainless steel subjected to in situ biaxial loading. ACTA Mater., 56, 4183-4199.

Meissonnier, F.T., Busso, E.P., O'Dowd, N.P., 2001. Finite element implementation of a generalised non- 
local rate-dependent crystallographic formulation for finite strains. Int. J. Plast., 17(4), 601-640.

Nakamachi, E., Tam. N.N., Morimoto, H., 2007. Multi-scale finite element analyses of sheet metals by using SEM-EBSD measured crystallographic RVE models. Int. J. Plast. 23(3), 450-489.

Oliver, E., Santisteban, J., James, J., Daymond, M., Dann, J., 2004. ENGIN-X user manual. ISIS Rutherford Laboratory.

Pang, J.W.L., Holden, T.M., Wright, J.S., Mason, T.E., 2000. The generation of intergranular strains in 309H stainless steel under uniaxial loading. ACTA Mater., 48, 1131-1140.

Quinta da Fonseca, J., Oliver, E.C., Bate, P.S., Withers, P.J., 2006. Evolution of intergranular stresses during in situ straining of IF steel with different grain sizes. Mater. Sci. Eng. A, 437, 26-32.

Rietveld, H.M., 1969. J. Appl. Crystallogr., 2, 65-71.

Santisteban, J.R., Daymond, M.R., Edwards, L., James, J.A., 2006. ENGIN-X: a third-generation neutron strain scanner. Journal of Applied Crystallography 39, 812-825.

Schmid, E., Siebel, G., 1931. Z. Elektrochem 37, 447.

Wong, S.L., Dawson, P.R., 2010. Influence of directional strength-to-stiffness on the elastic-plastic transition of fcc polycrystals under uniaxial tensile loading. Acta Materialia, 58, 1658-1678. 


\section{Appendix. Lattice plane modulus of single crystal under uniaxial loading}

Here, we follow the approach of Norris (2006) to investigate the transverse and longitudinal moduli in a cubic single crystal. The longitudinal modulus is the modulus along the loading direction and the transverse modulus is the modulus normal to the loading direction. The elastic stress-strain relationship for a cubic material can be written in the material frame as

$$
\left[\begin{array}{c}
\sigma_{11} \\
\sigma_{22} \\
\sigma_{33} \\
\sigma_{12} \\
\sigma_{13} \\
\sigma_{23}
\end{array}\right]=\left[\begin{array}{cccccc}
C_{11} & C_{12} & C_{12} & 0 & 0 & 0 \\
C_{12} & C_{11} & C_{12} & 0 & 0 & 0 \\
C_{12} & C_{12} & C_{11} & 0 & 0 & 0 \\
0 & 0 & 0 & C_{44} & 0 & 0 \\
0 & 0 & 0 & 0 & C_{44} & 0 \\
0 & 0 & 0 & 0 & 0 & C_{44}
\end{array}\right]\left[\begin{array}{c}
\varepsilon_{11} \\
\varepsilon_{22} \\
\varepsilon_{33} \\
\gamma_{12} \\
\gamma_{13} \\
\gamma_{23}
\end{array}\right]
$$

where $C_{11}, C_{12}$ and $C_{44}$ are the elastic stiffness constants of the crystal. Alternatively, the stress-strain relationship can be written in terms of the compliance matrix as

$$
\left[\begin{array}{l}
\varepsilon_{11} \\
\varepsilon_{22} \\
\varepsilon_{33} \\
\gamma_{12} \\
\gamma_{13} \\
\gamma_{23}
\end{array}\right]=\left[\begin{array}{cccccc}
1 / E & -\nu / E & -\nu / E & 0 & 0 & 0 \\
-\nu / E & 1 / E & -\nu / E & 0 & 0 & 0 \\
-\nu / E & -\nu / E & 1 / E & 0 & 0 & 0 \\
0 & 0 & 0 & 1 / G & 0 & 0 \\
0 & 0 & 0 & 0 & 1 / G & 0 \\
0 & 0 & 0 & 0 & 0 & 1 / G
\end{array}\right]\left[\begin{array}{l}
\sigma_{11} \\
\sigma_{22} \\
\sigma_{33} \\
\sigma_{12} \\
\sigma_{13} \\
\sigma_{23}
\end{array}\right],
$$

where $E, \nu$ and $G$ are the cubic plane modulus, Poisson ratio and shear modulus, respectively. These constants are functions of the elastic stiffness constants as given by

$$
\begin{aligned}
E & =\left(C_{11}+2 C_{12}\right)\left(C_{11}-C_{12}\right) /\left(C_{11}+C_{12}\right), \\
\nu & =C_{12} /\left(C_{11}+C_{12}\right), \\
G & =C_{44} .
\end{aligned}
$$

Defining the uniaxial load as being applied parallel to the normal of a particular $(h k l)$ lattice plane (see Fig. A1), the applied stress, $\boldsymbol{\sigma}$, can be written as

$$
\boldsymbol{\sigma}=\sigma_{0} \boldsymbol{e}_{h k l} \otimes \boldsymbol{e}_{h k l},
$$

where $\sigma_{0}$ is the stress magnitude and $\boldsymbol{e}_{h k l}$ indicates the unit normal vector to the ( $h k l$ ( lattice plane, which can be expressed in terms of a cubic frame basis $\left(\boldsymbol{e}_{1}, \boldsymbol{e}_{2}\right.$ and $\left.\boldsymbol{e}_{3}\right)$ as

$$
\boldsymbol{e}_{h k l}=\left(h \boldsymbol{e}_{1}+k \boldsymbol{e}_{2}+l \boldsymbol{e}_{3}\right) / \sqrt{h^{2}+k^{2}+l^{2}} .
$$

Thus the stress components in the cubic frame can be calculated by substituting Eqn. A5 into Eqn. A-4. The cubic frame components of the elastic strain tensor, $\varepsilon$, are then determined by the linear stress-strain relationship, Eqn. A-2.

To evaluate the lattice stretch of an arbitrary $\left(h_{1} k_{1} l_{1}\right)$ lattice plane, $\varepsilon_{h_{1} k_{1} l_{1}}$, a tensor transformation leads to

$$
\varepsilon_{h_{1} k_{1} l_{1}}=\boldsymbol{\varepsilon}:\left(\boldsymbol{e}_{h_{1} k_{1} l_{1}} \otimes \boldsymbol{e}_{h_{1} k_{1} l_{1}}\right) .
$$


Eqn. A-6 indicates that the $\left(h_{1} k_{1} l_{1}\right)$ lattice plane stretch is a linear function of the applied stress magnitude, $\sigma_{0}$.

For the interpretation of the ND results, it is of interest to examine the specific cases of longitudinal and transverse lattice stretches (see Fig. 1(a)). The longitudinal lattice strain is the strain in the direction of loading, $h_{1}=h, k_{1}=k$ and $l_{1}=l$ in Eqn. (A-6) and the transverse strain is the strain in the orthogonal direction (strain normal to the plane $\left(h_{1} k_{1} l_{1}\right)$ in Fig. A1 for the loading direction indicated). Note also that in an ND measurement, results are obtained as averages for grain families, i.e. the ND technique does not distinguish between, e.g. a [220] orientated grain and a [2 $\overline{2} 0]$ orientated grain.

For the longitudinal strain, $h_{1}=h, k_{1}=k$ and $l_{1}=l$, Eqn. A-6 leads to

$$
\begin{aligned}
\varepsilon_{h k l}^{L} & =\sigma_{0} / E_{h k l}^{L}, \\
E_{h k l}^{L} & =E\left[1-(2+2 \nu)(1-\alpha) f^{L}\right]^{-1}, \\
f^{L} & =\left(h^{2} k^{2}+h^{2} l^{2}+k^{2} l^{2}\right) /\left(h^{2}+k^{2}+l^{2}\right)^{2},
\end{aligned}
$$

where $\varepsilon_{h k l}^{L}$ and $E_{h k l}^{L}$ indicate $(h k l)$ longitudinal lattice strain and longitudinal modulus, respectively, and $\alpha$ defines the material anisotropy ratio given by

$$
\alpha=\left(C_{11}-C_{12}\right) /\left(2 C_{44}\right)
$$

Using Eqn.(A-7) the values of longitudinal lattice plane modulus shown in Table 4 are readily obtained.

For the transverse lattice strain, the $(h k l)$ and $\left(h_{1} k_{1} l_{1}\right)$ lattice planes are orthogonal (see Fig. A1). So the following constraint condition holds

$$
h_{1} h+k_{1} k+l_{1} l=0 .
$$

Thus, from Eqn. A-6 and applying the constraint condition, Eqn. A-9, the transverse $\left(h_{1} k_{1} l_{1}\right)$ lattice strain, $\varepsilon_{h_{1} k_{1} l_{1}}^{T}$, is given by

$$
\begin{aligned}
\varepsilon_{h_{1} k_{1} l_{1}}^{T} & =\sigma_{0} / E_{h_{1} k_{1} l_{1}}^{T}, \\
E_{h_{1} k_{1} l_{1}}^{T} & =-E\left[\nu-(1+\nu)(1-\alpha) f^{T}\right]^{-1}, \\
f^{T} & =\left(h^{2} h_{1}^{2}+k^{2} k_{1}^{2}+l^{2} l_{1}^{2}\right) /\left(h^{2}+k^{2}+l^{2}\right) /\left(h_{1}^{2}+k_{1}^{2}+l_{1}^{2}\right),
\end{aligned}
$$

where $E_{h_{1} k_{1} l_{1}}^{T}$ is the $\left\{h_{1} k_{1} l_{1}\right\}$ transverse lattice modulus. The factor, $f^{T}$ depends on the loading direction $\boldsymbol{e}_{h k l}$ and its orthogonal direction $\boldsymbol{e}_{h_{1} k_{1} l_{1}}$ and thus a range of values are possible for $E_{h_{1} k_{1} l_{1}}^{T}$ from Eq.A-10b. However, the constraint conditions, Eq. A-9, puts limits on the possible values of $E_{h_{1} k_{1} l_{1}}^{T}$. These limits can be expressed through the extreme values of $f^{T}$ following the approach of Norris (2006). Based on the result in Norris (2006) the extreme values of $f^{T}$ are,

$$
f_{\max , \min }^{T}=\frac{\left(h_{1}^{2} k_{1}^{2}+k_{1}^{2} l_{1}^{2}+h_{1}^{2} l_{1}^{2}\right) \pm \sqrt{\left(h_{1}^{2} k_{1}^{2}+k_{1}^{2} l_{1}^{2}+h_{1}^{2} l_{1}^{2}\right)^{2}-3 h_{1}^{2} k_{1}^{2} l_{1}^{2}\left(h_{1}^{2}+k_{1}^{2}+l_{1}^{2}\right)}}{\left(h_{1}^{2}+k_{1}^{2}+l_{1}^{2}\right)^{2}}
$$


Note that a unique value of $f^{T}$ is obtained if the term under the square root is zero. Examples of extreme values are given for the $\left(h_{1} k_{1} l_{1}\right)$ planes as follows

$$
\begin{aligned}
& \left(h_{1} k_{1} l_{1}\right)=(220) \Rightarrow 0 \leqslant f^{T} \leqslant 1 / 2, \\
& \left(h_{1} k_{1} l_{1}\right)=(311) \Rightarrow 1 / 11 \leqslant f^{T} \leqslant 27 / 121 .
\end{aligned}
$$

For all other relevant cases, $f^{T}$ is unique.

By substituting the values of $f^{T}$ obtained from Eqns. (A-11) into Eqn. (A-10b), the values of the transverse lattice plane modulus shown in Table 4 are obtained. Note that this result is analogous to the result of Norris (2006) which showed that the Poisson ratio of a single cubic crystal is not unique but depends on the crystal orientation relative to the loading axis.

The discussion here relates to a single crystal of arbitrary orientation relative to an applied uniaxial loading direction. The significance of the result for a polycrystal is that when determine the transverse strains for a grain family, the transverse modulus of individual grains may fall anywhere within the range provided in Table 4 (ignoring the effect of local grain interaction). Thus, the transverse strain is expected to be sensitive to the orientation distribution (texture) of the polycrystal, and may vary from specimen to specimen, while the longitudinal strain is not expected to show such sensitivity.

\section{References}

Norris, A.N., 2006. Poisson's ratio in cubic materials. Proc. R. Soc. A, 462, 3385-3405. 
Table 1: Chemical composition in wt $\%$ for the $316 \mathrm{H}$ stainless steel.

\begin{tabular}{ccccccccccc}
\hline Fe & $\mathrm{Cr}$ & $\mathrm{Ni}$ & $\mathrm{Mo}$ & $\mathrm{Mn}$ & $\mathrm{Si}$ & $\mathrm{Co}$ & $\mathrm{C}$ & $\mathrm{S}$ & $\mathrm{P}$ & $\mathrm{B}$ \\
\hline Balance & 17.18 & 10.92 & 2 & 1.5 & 0.32 & 0.08 & 0.055 & 0.014 & 0.021 & 0.003 \\
\hline
\end{tabular}

Table 2: Elastic properties of $316 \mathrm{H}$ stainless steel at room temperature.

\begin{tabular}{cccc}
\hline$C_{11}$ & $C_{12}$ & $C_{44}$ & $\mu_{0}$ \\
$\mathrm{GPa}$ & $\mathrm{GPa}$ & $\mathrm{GPa}$ & $\mathrm{GPa}$ \\
\hline $232^{1}$ & $154^{1}$ & $118^{1}$ & 127.6 \\
\hline${ }^{1}$ Data & from & Daymond and \\
Bouchard (2006)
\end{tabular}

Table 3: Flow rule and slip resistance parameters at room temperature.

\begin{tabular}{cccccccc}
\hline$\dot{\gamma}_{0}$ & $p$ & $q$ & $\mathrm{Q}$ & $\begin{array}{c}\hat{\tau}_{0} \\
\mathrm{MPa}\end{array}$ & $\begin{array}{c}S_{0} \\
\mathrm{MPa}\end{array}$ & $\begin{array}{c}S_{\text {sat }} \\
\mathrm{MPa}\end{array}$ & $\begin{array}{c}h_{s} \\
\mathrm{MPa}\end{array}$ \\
\hline 450 & 1.0 & 1.9 & 117.4 & 144 & 2.22 & 220.3 & 1087 \\
\hline
\end{tabular}


Table 4: Lattice plane modulus under uniaxial loading.

\begin{tabular}{ccccc}
\hline Lattice strain & $\{h k l\}$ & $\begin{array}{c}\text { Measurements } \\
\text { GPa }\end{array}$ & $\begin{array}{c}\text { Predictions } \\
\text { GPa }\end{array}$ & $\begin{array}{c}\text { Single } \text { crystal }^{1} \\
\text { GPa }\end{array}$ \\
\hline Longitudinal & $\{200\}$ & $163 \pm 4$ & $153 \pm 1$ & 110 \\
& $\{111\}$ & $251 \pm 6$ & $251 \pm 11$ & 289 \\
& $\{220\}$ & $206 \pm 1$ & $219 \pm 15$ & 205 \\
Transverse & $\{311\}$ & $196 \pm 6$ & $168 \pm 7$ & 155 \\
& $\{200\}$ & $-488 \pm 34$ & $-381 \pm 29$ & -275 \\
& $\{111\}$ & $-1004 \pm 57$ & $-974 \pm 130$ & -1255 \\
& $\{220\}$ & $-770 \pm 25$ & {$[-1283,-490]^{2}$} & {$[-\infty,-275] \bigcup[1618,+\infty]$} \\
\hline
\end{tabular}

${ }^{1}$ Theoretical predictions are derived in the Appendix, based on experimental data from Daymond and Bouchard (2006).

${ }^{2}$ Square brackets indicate ranges over 6 simulations. 


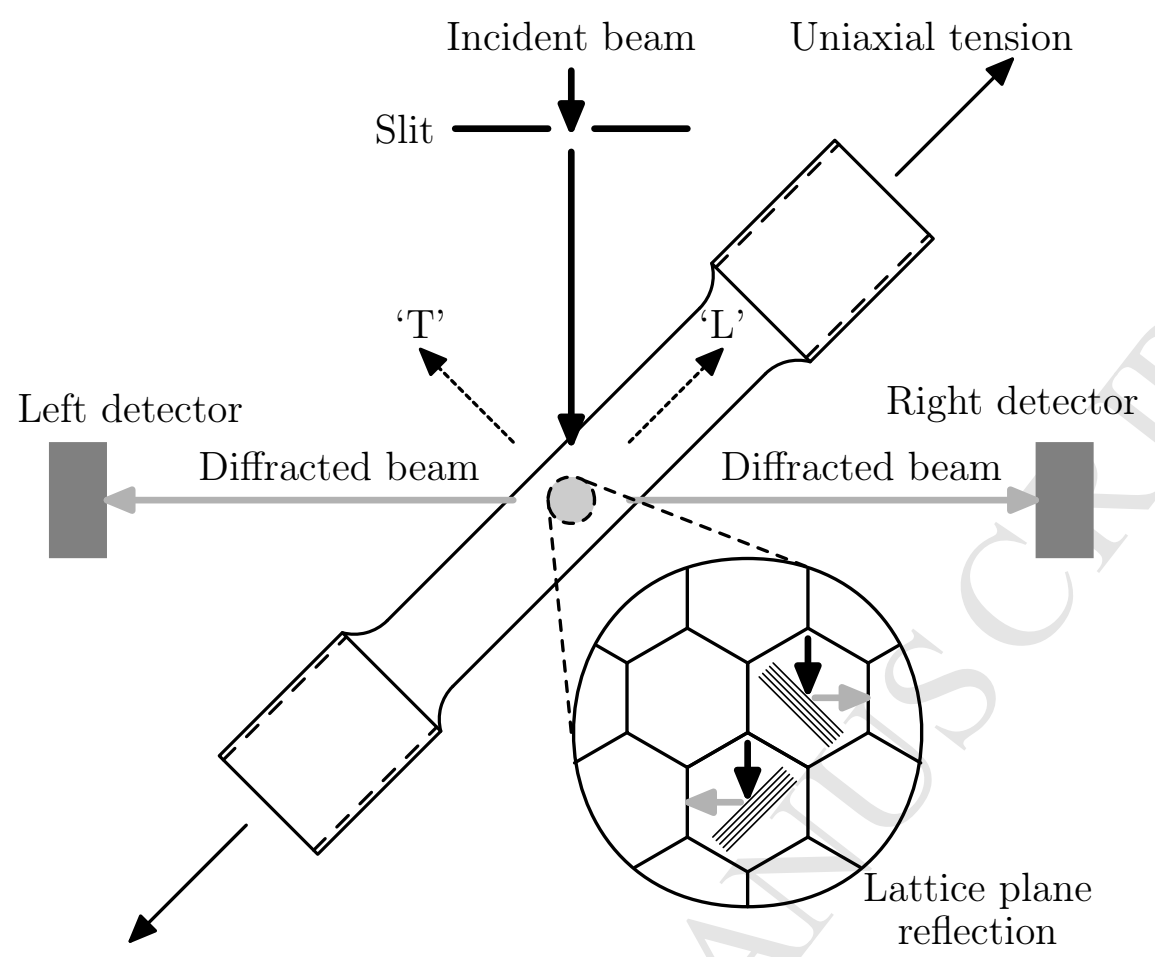

(a)

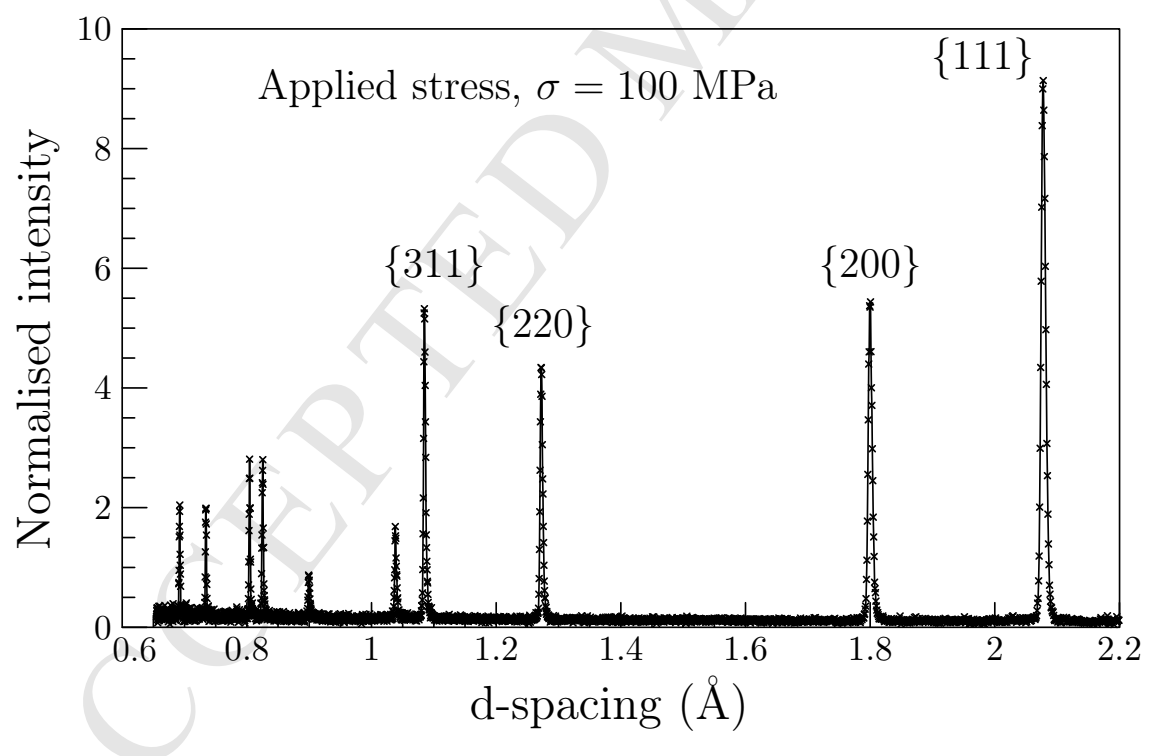

(b)

Figure 1: (a) Schematic illustration of ENGIN-X at ISIS, UK. 'T' and 'L' indicate transverse and longitudinal scattering vectors, respectively. (b) Typical spectra recorded from ENGIN-X during uniaxial loading. 


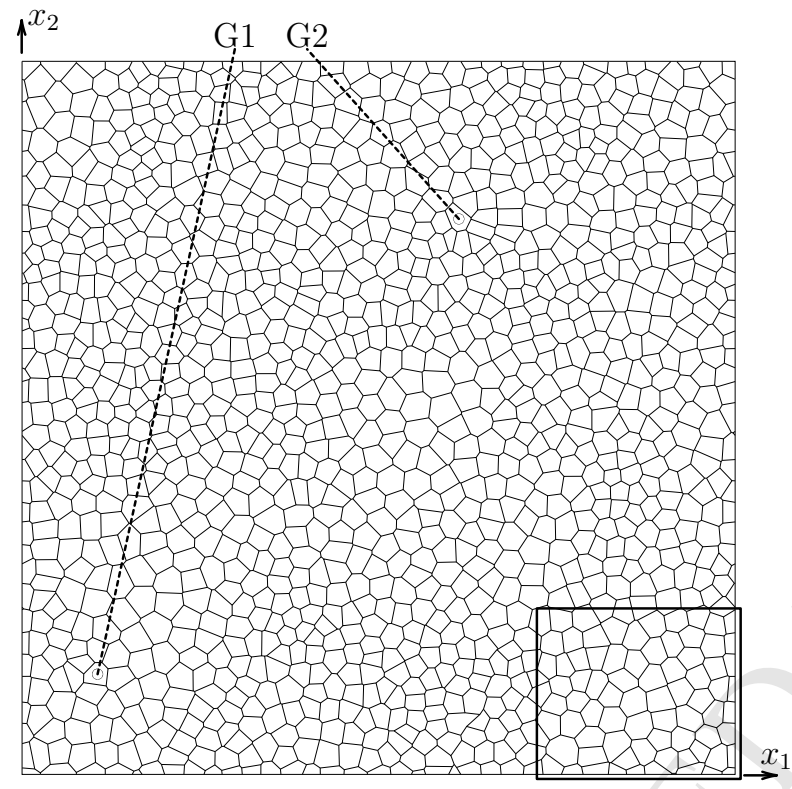

(a)

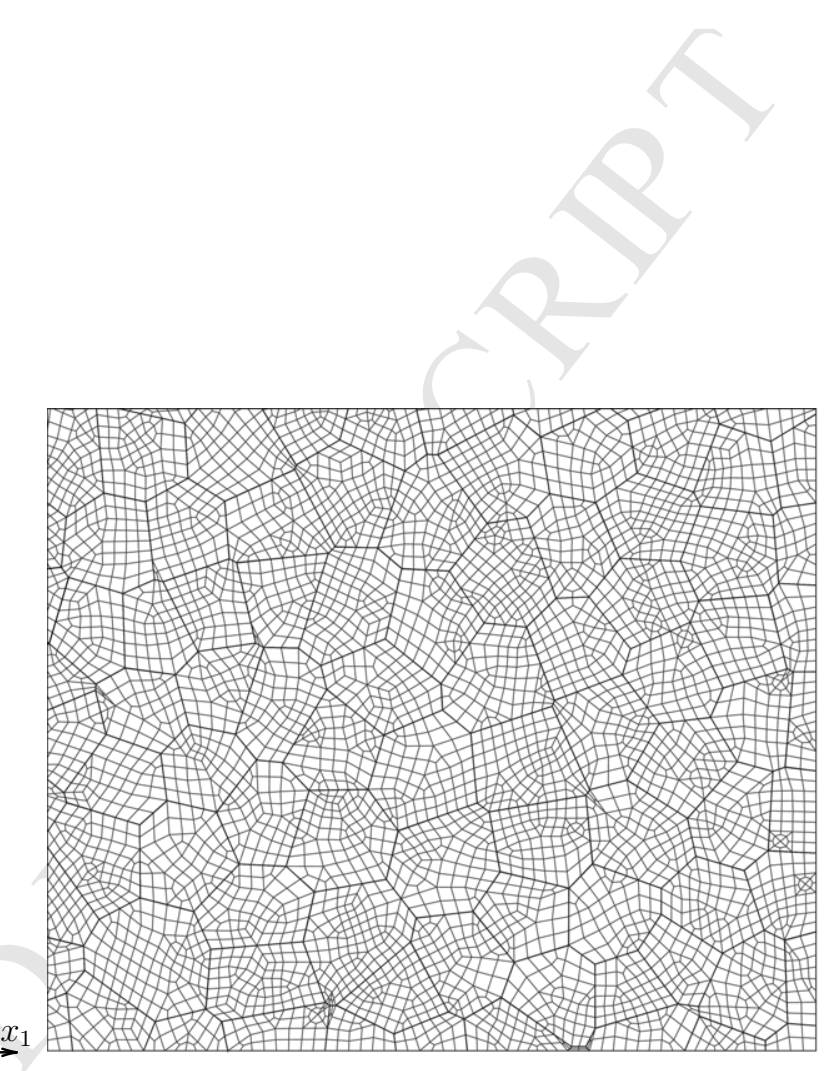

(b)

Figure 2: Representative volume element (RVE) with random grain shape and finite element mesh: (a) RVE topology and (b) finite element mesh of right-bottom corner (the grains labelled G1 and G2 in Fig. 2(a) will be discussed in Section 4.3). 


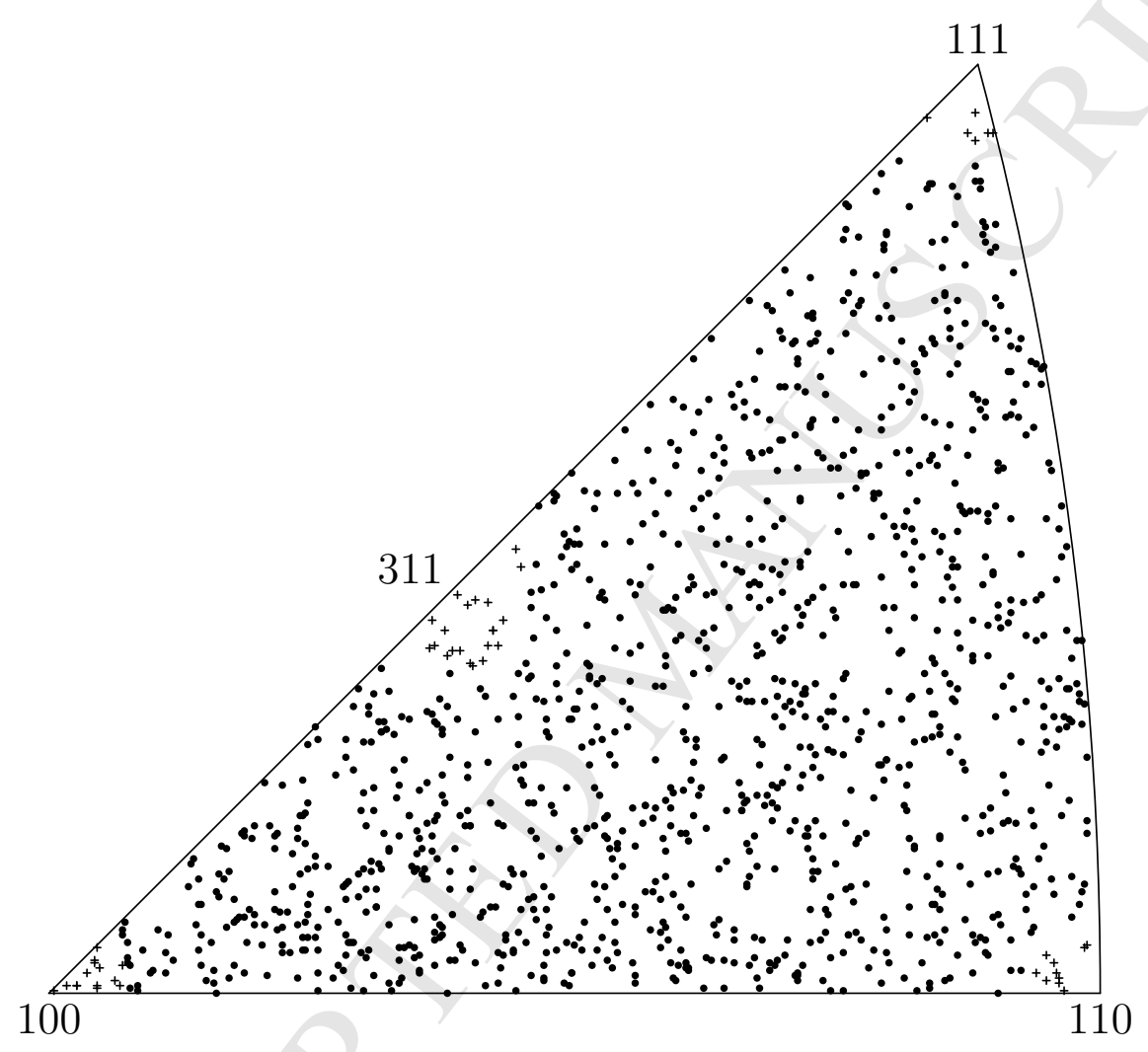

Figure 3: Inverse pole figure in loading direction: the crosses represent individual grains which are averaged to represent the response of each grain family. 


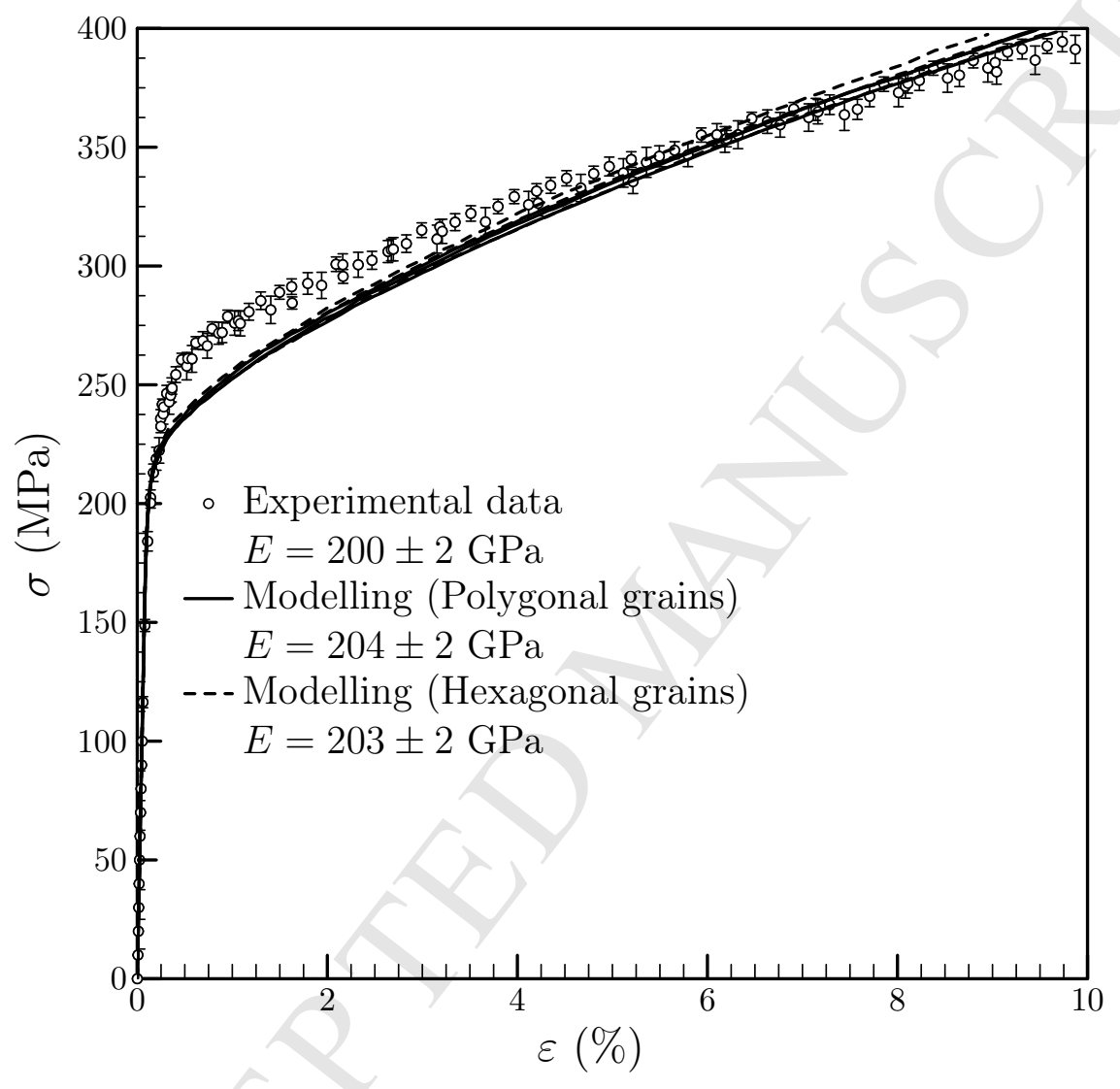

Figure 4: Macroscopic stress strain curve. 


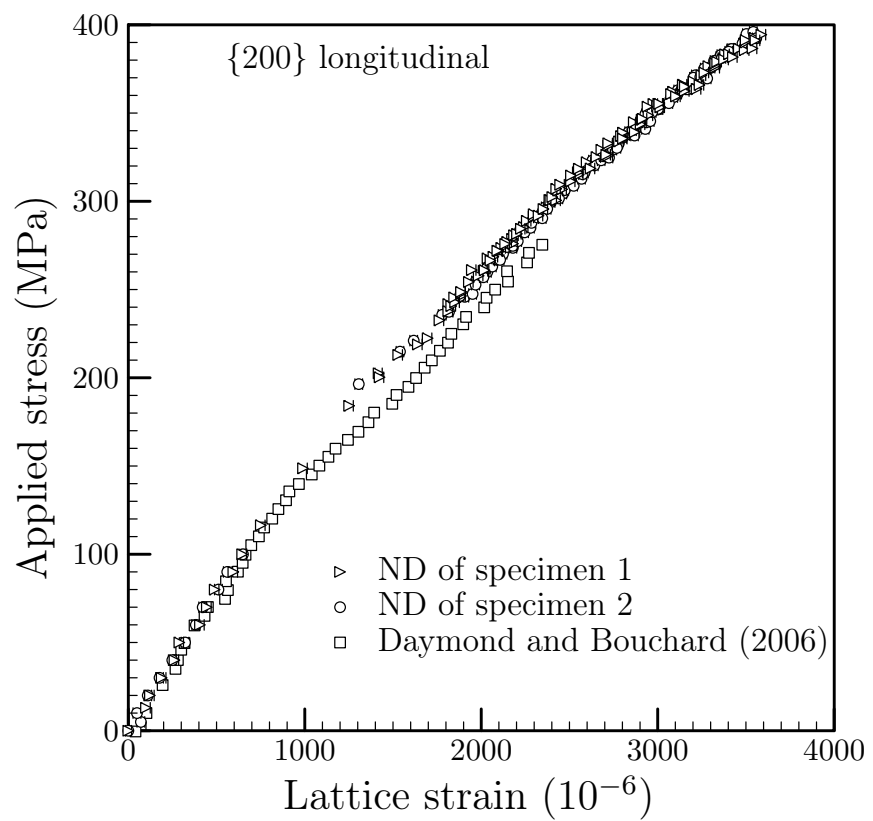

(a)

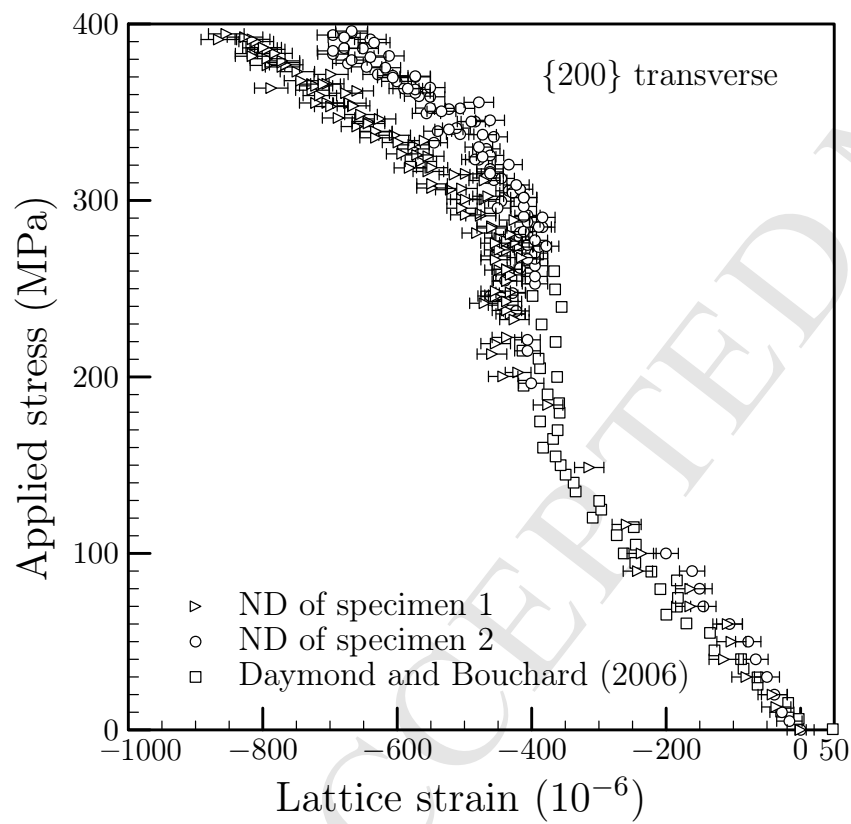

(c)

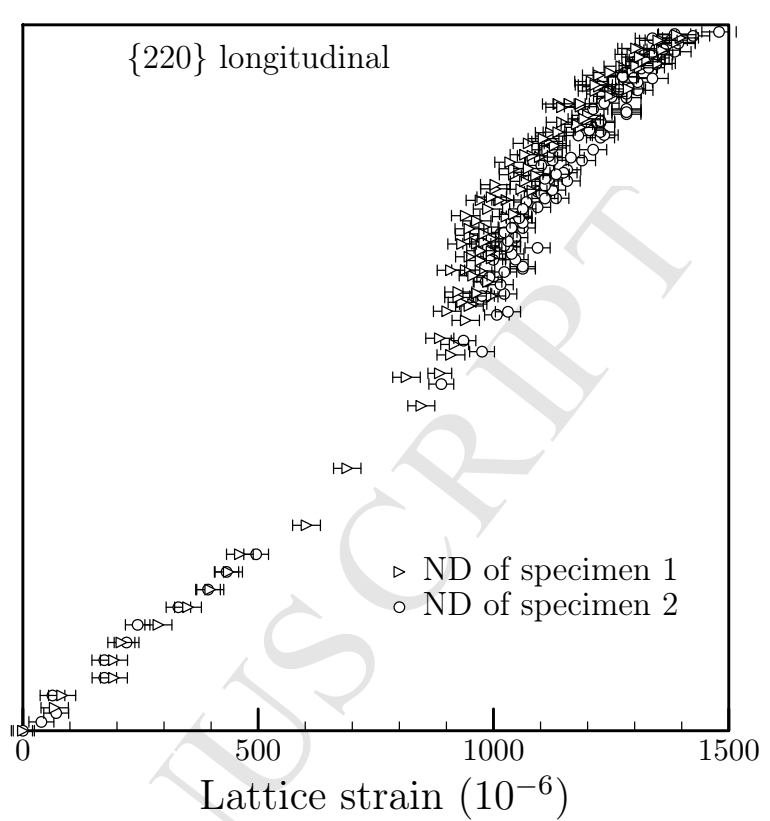

(b)

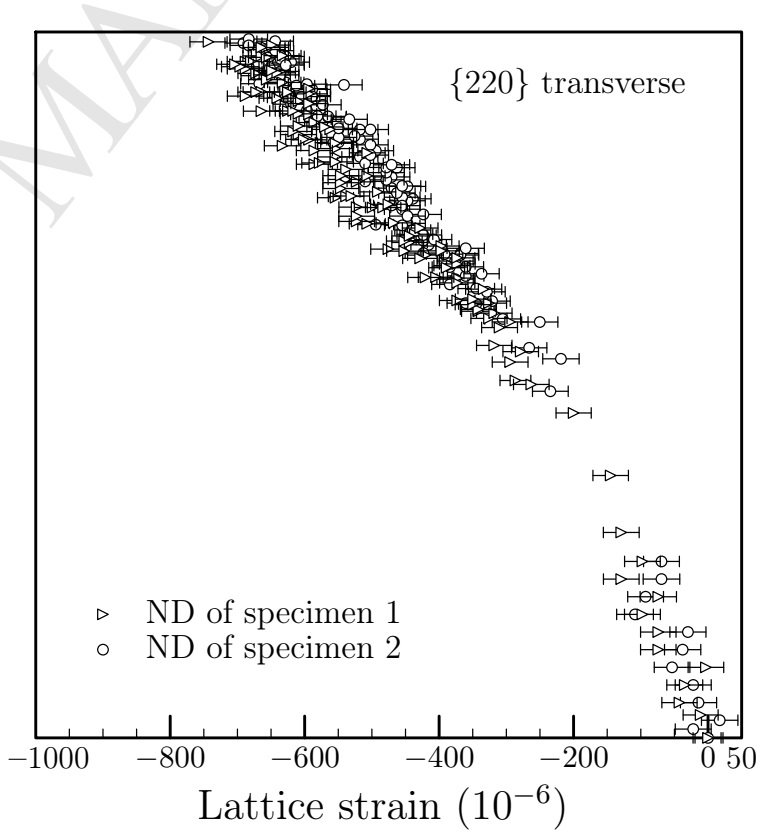

(d)

Figure 5: Lattice strain versus stress applied from experimental data: (a) longitudinal $\{200\}$ lattice plane, (b) longitudinal $\{220\}$ lattice plane, (c) transverse $\{200\}$ lattice plane and (d) transverse $\{220\}$ lattice plane. 


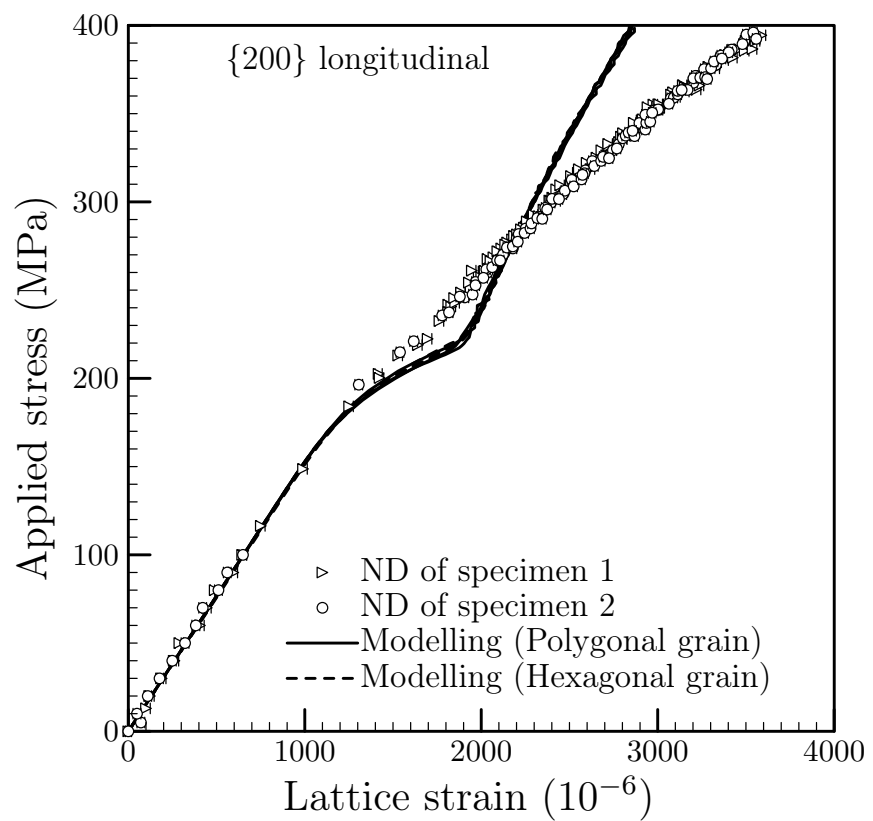

(a)

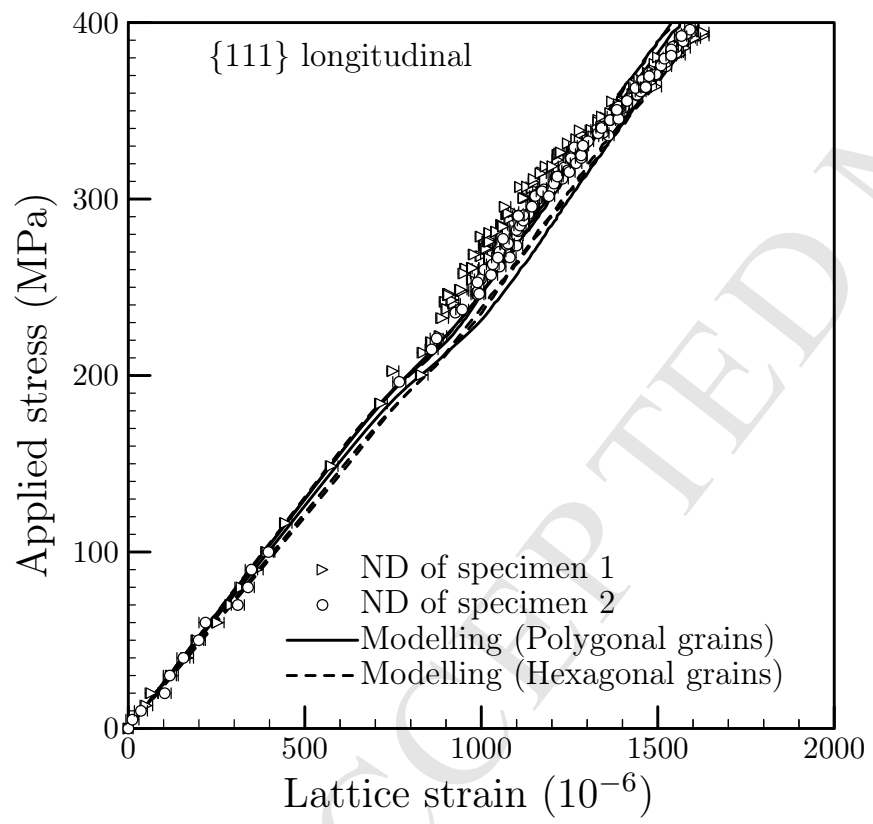

(c)

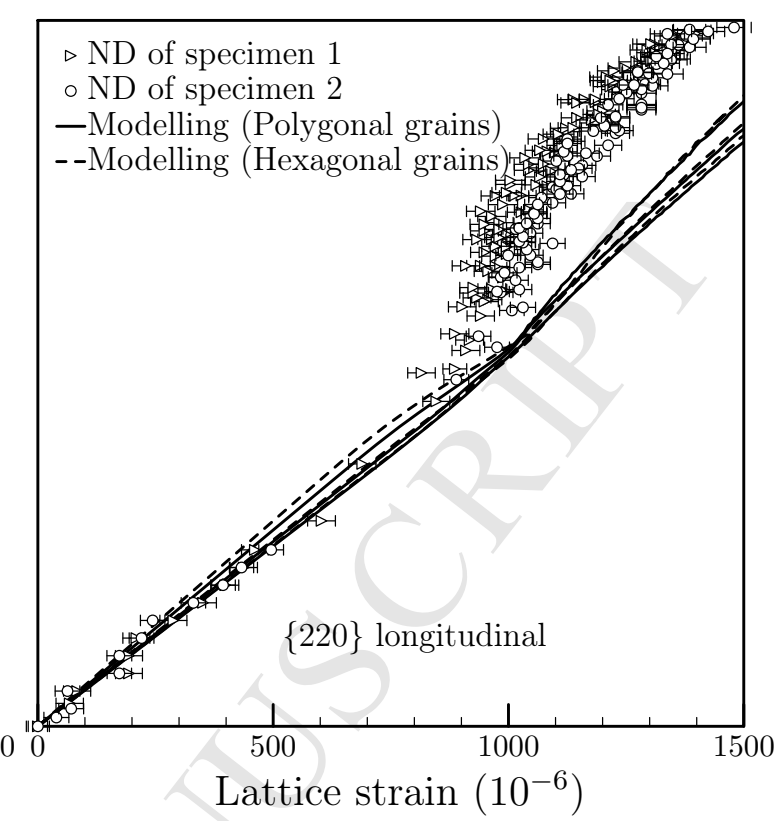

(b)

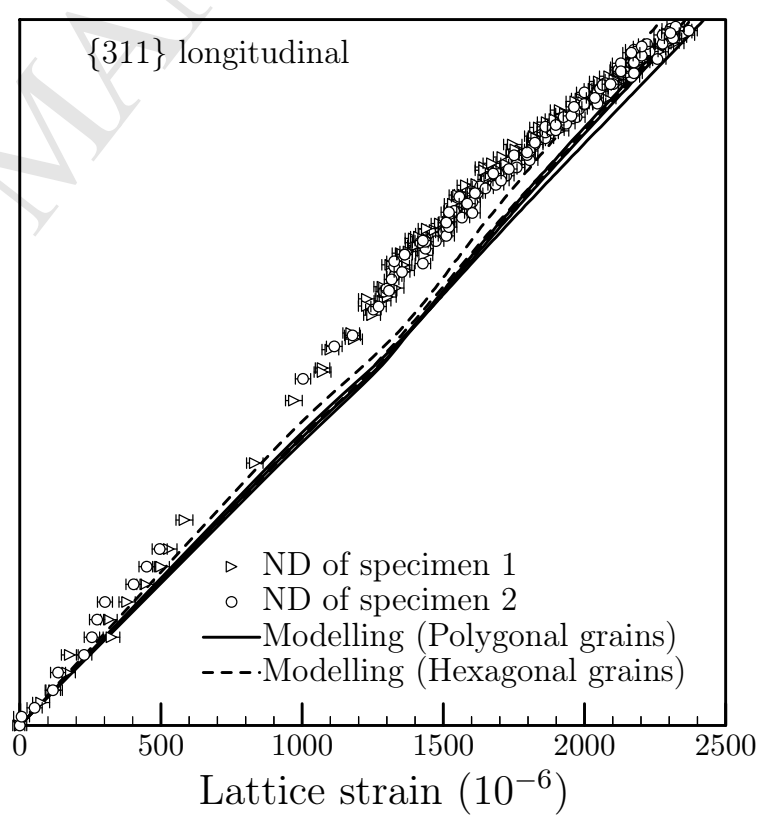

(d)

Figure 6: Longitudinal lattice strain versus applied stress from measurements and modelling predictions: (a) $\{111\}$ lattice plane, (b) $\{200\}$ lattice plane, (c) $\{220\}$ lattice plane and (d) $\{311\}$ lattice plane. (ND Neutron Diffraction) 


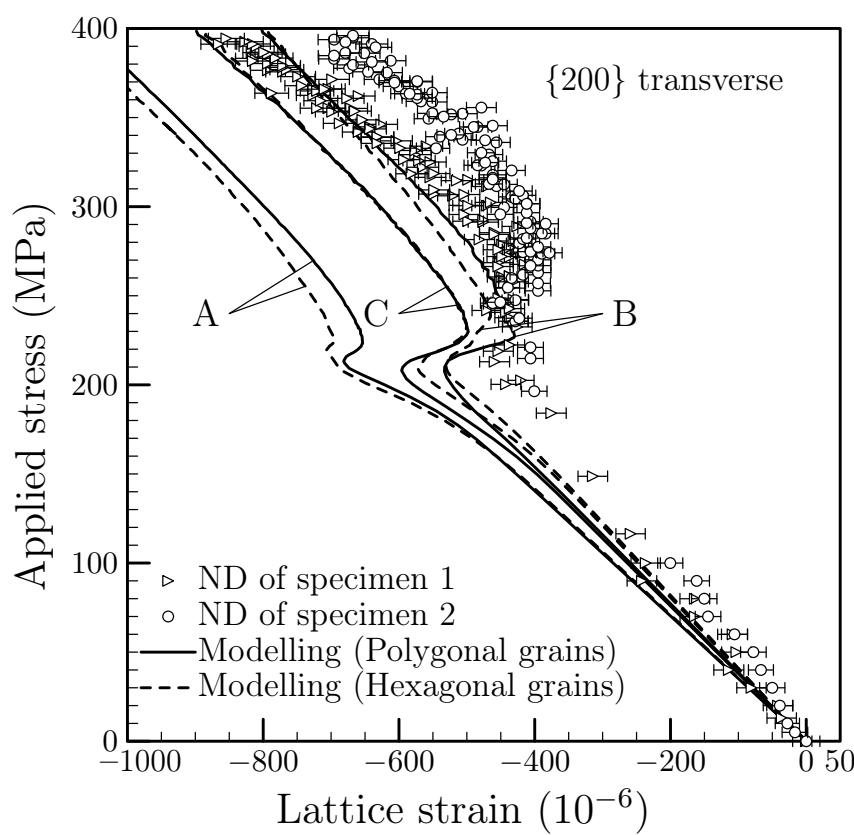

(a)

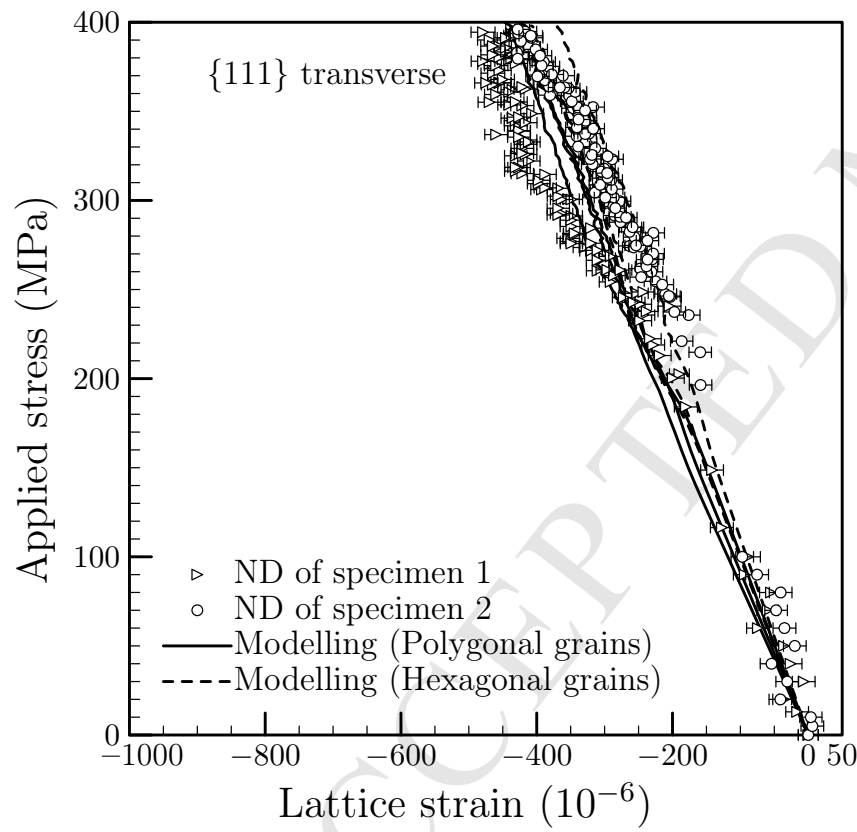

(c)

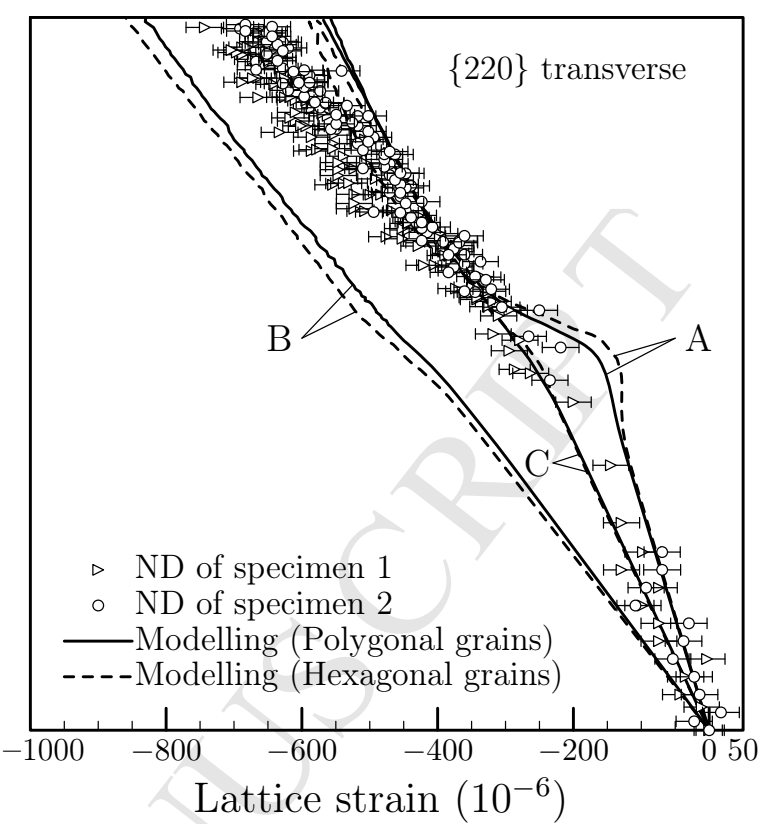

(b)

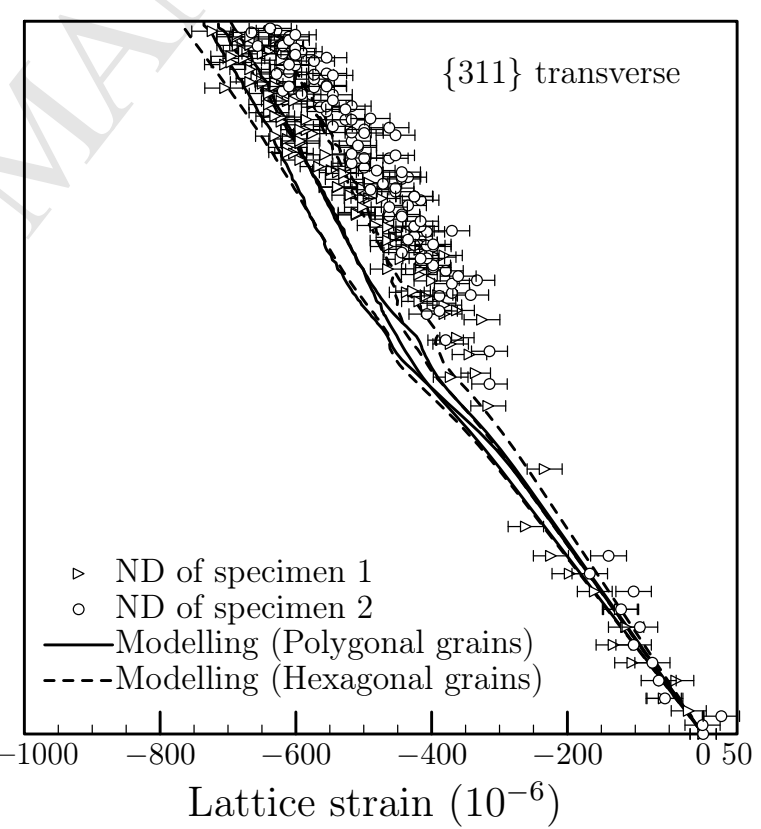

(d)

Figure 7: Transverse lattice strain versus stress applied from measurements and modelling predictions: (a) $\{111\}$ lattice plane, (b) $\{200\}$ lattice plane, (c) $\{220\}$ lattice plane and (d) $\{311\}$ lattice plane ('A', 'B' and ' $\mathrm{C}$ ' indicate modelling results for three distinctive orientation maps). 


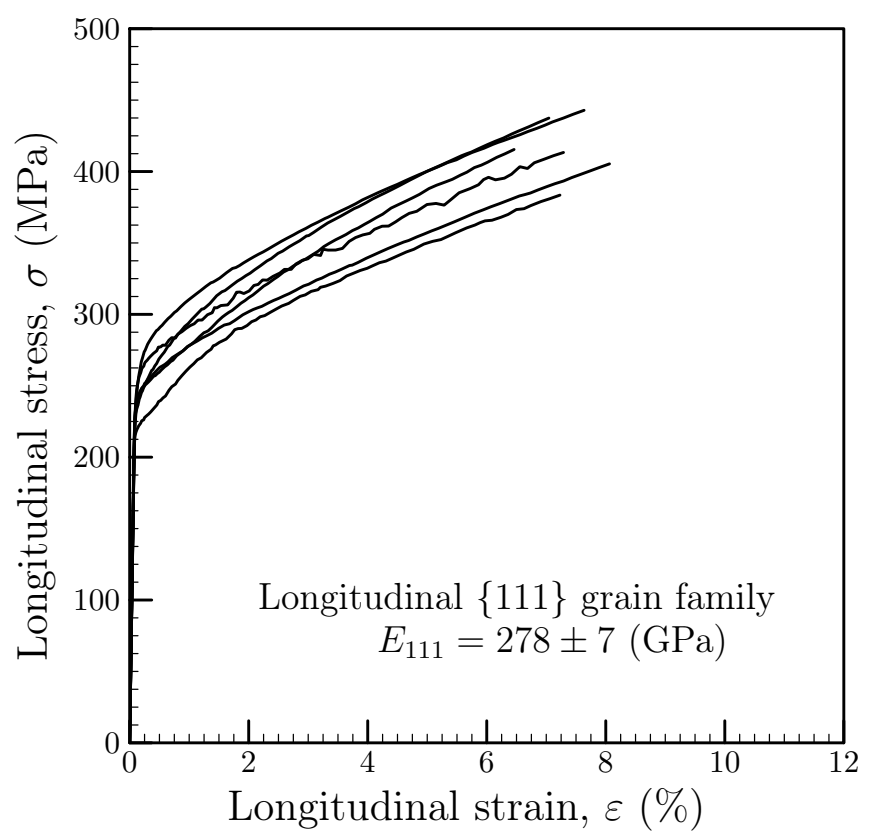

(a)

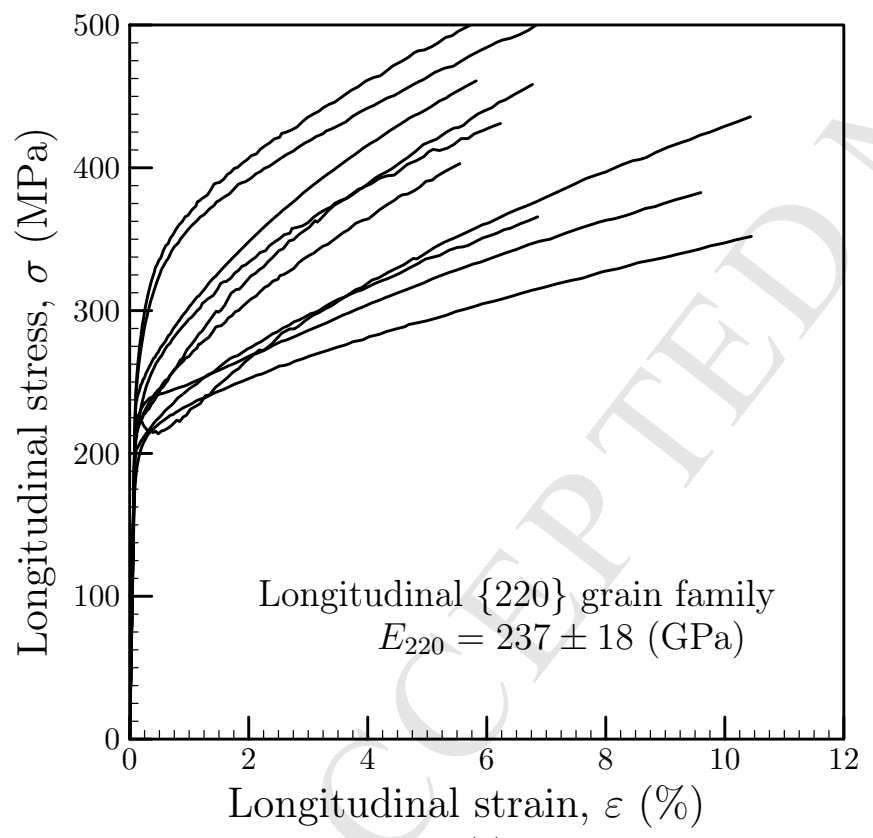

(c)

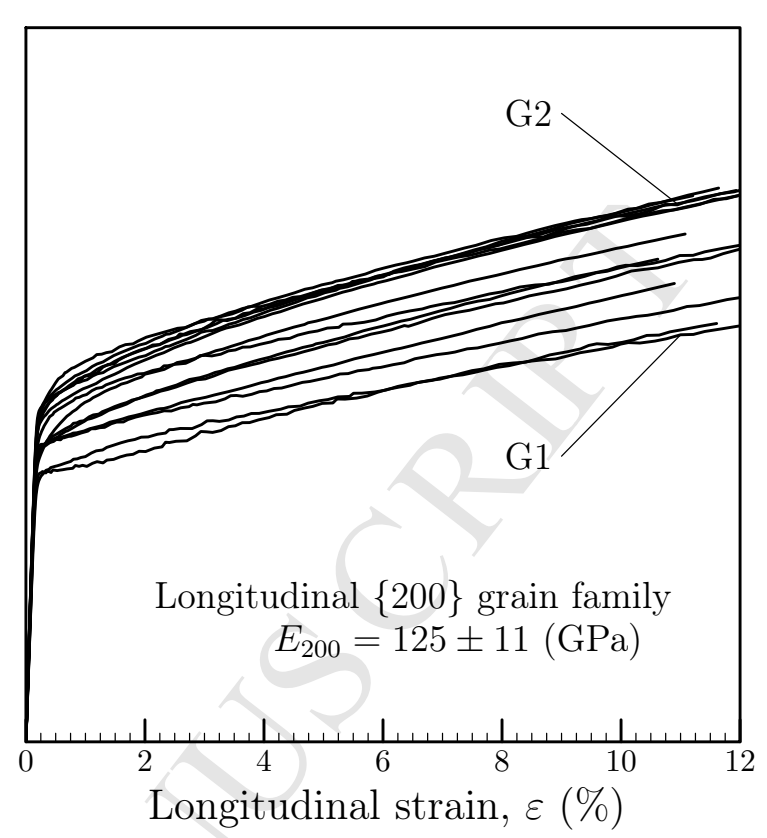

(b)

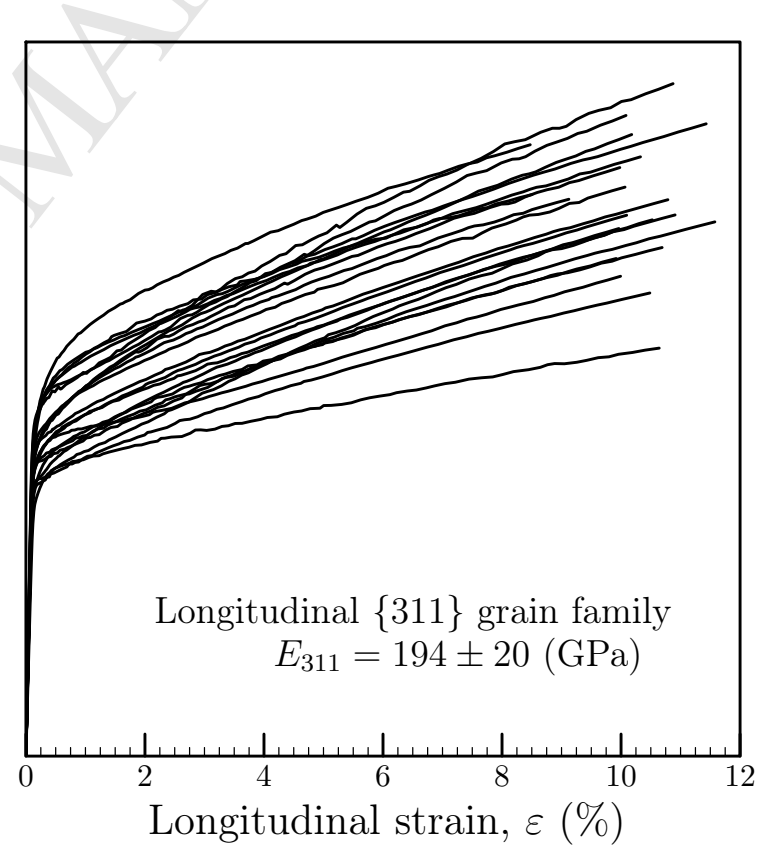

(d)

Figure 8: Longitudinal stress strain curves within different grain families for orientation map 'A': a) $\{111\}$ lattice plane, (b) $\{200\}$ lattice plane, (c) $\{220\}$ lattice plane and (d) $\{311\}$ lattice plane. 

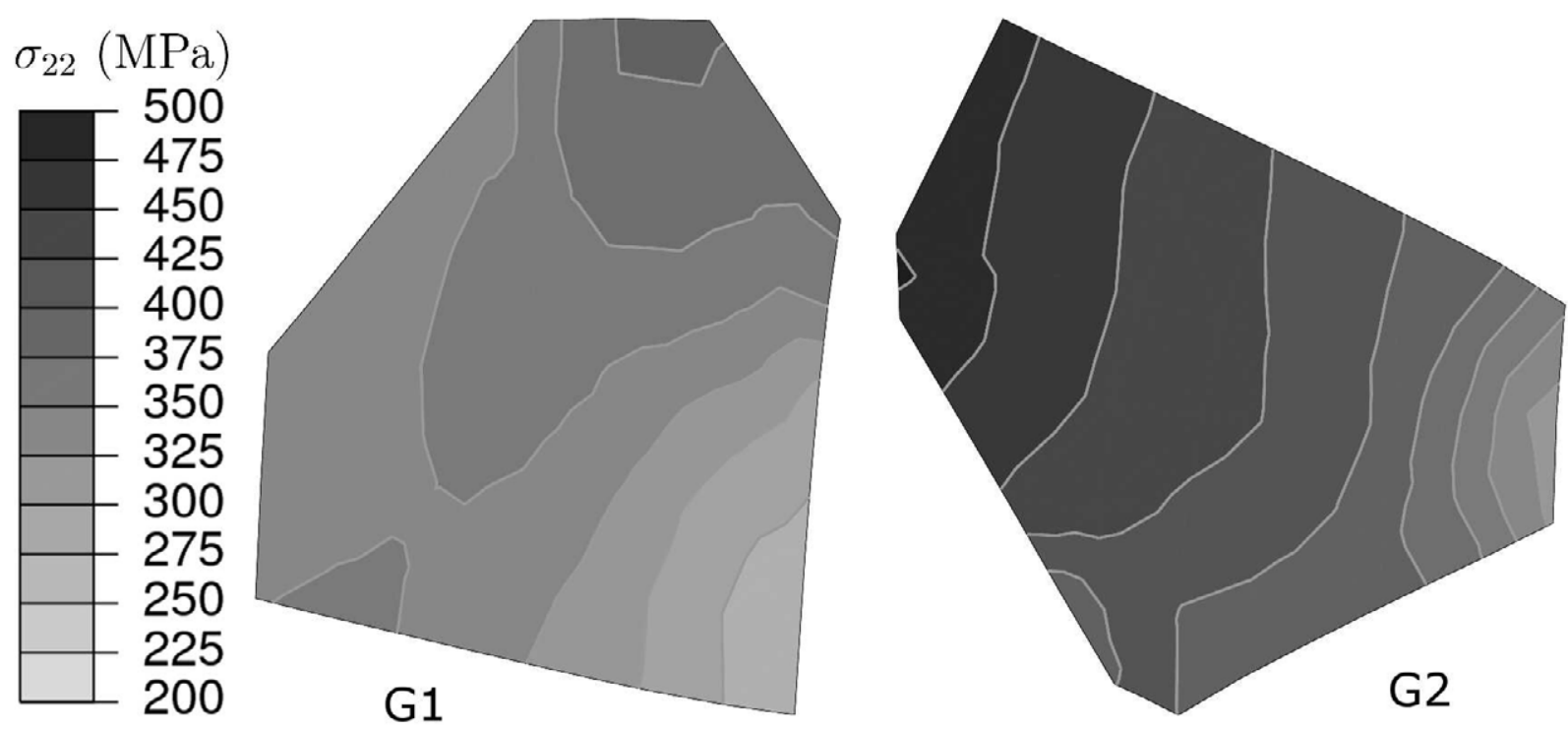

Figure 9: Intragranular stress distribution of $\{200\}$ longitudinal grain family (random orientation set 'A') at $400 \mathrm{MPa}$ of applied stress. Grains are identified by G1 and G2 in Fig. 2(a). 


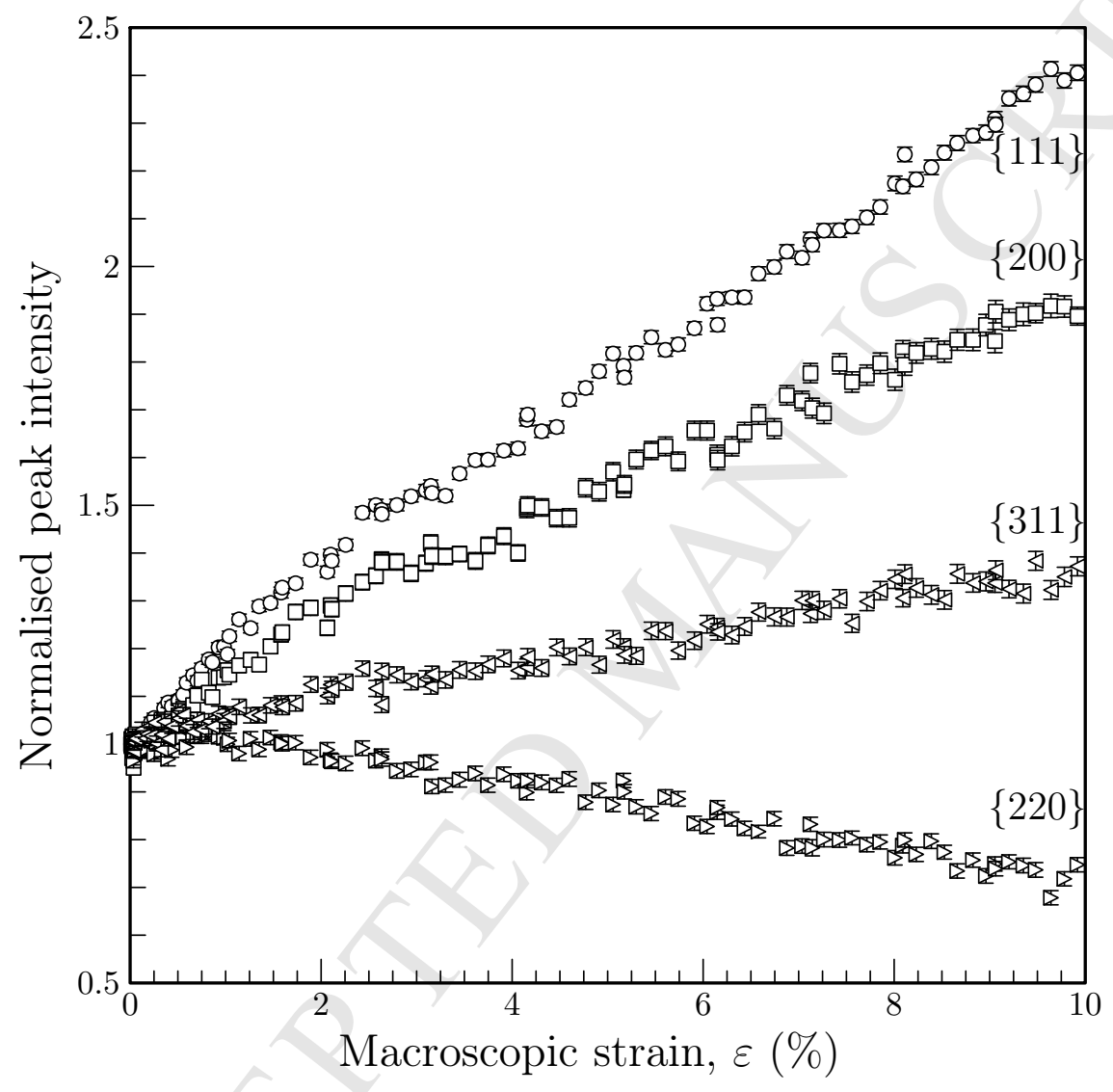

Figure 10: Peak intensity evolution during uniaxial tensile deformation from ND data of the right detector. 


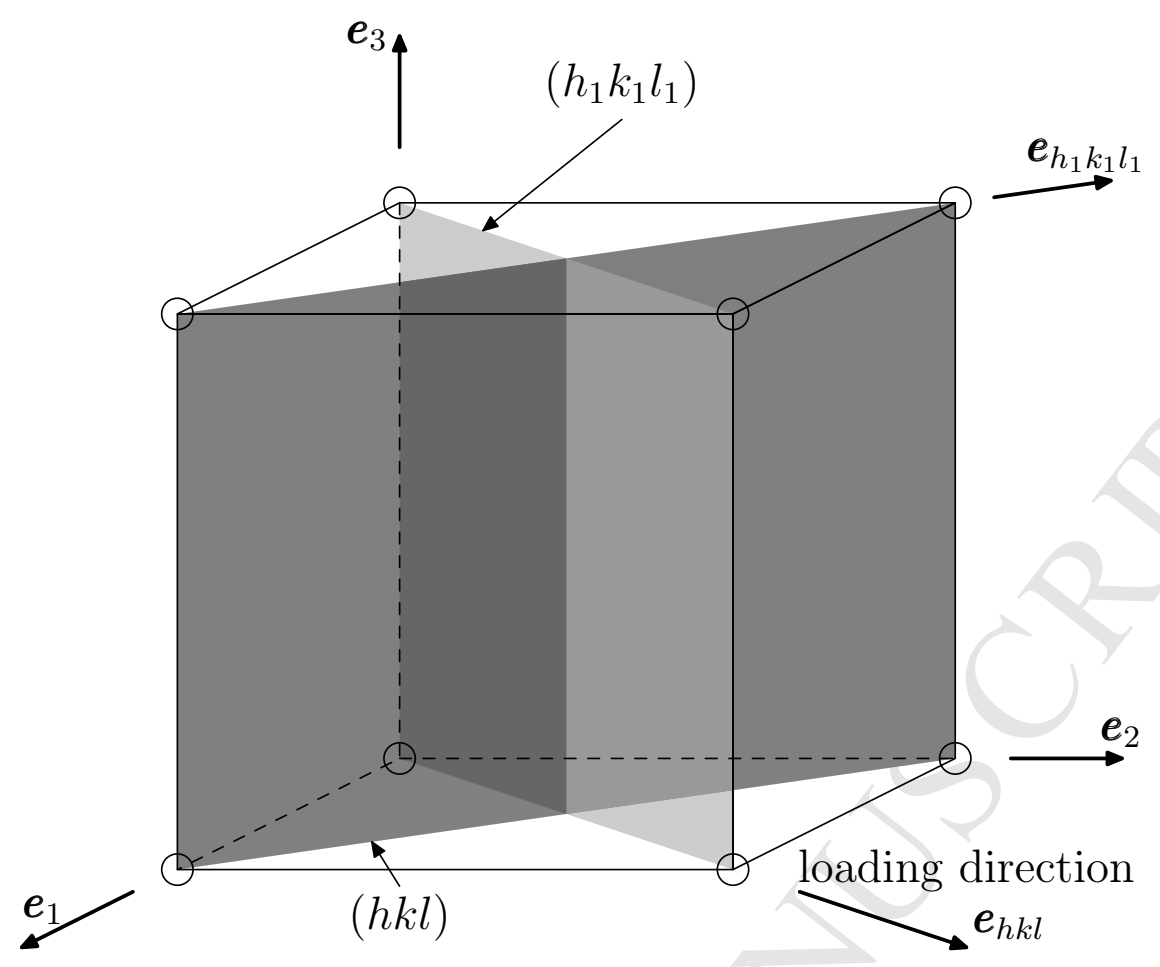

Figure A1, Illustration of the lattice planes of a single cubic crystal where $\boldsymbol{e}_{h k l}$ is the uniaxial loading direction. Here the plane $(h k l)$ is the $(110)$ plane and $\left(h_{1} k_{1} l_{1}\right)$ is the $(\overline{1} 10)$ plane. 
Left detector

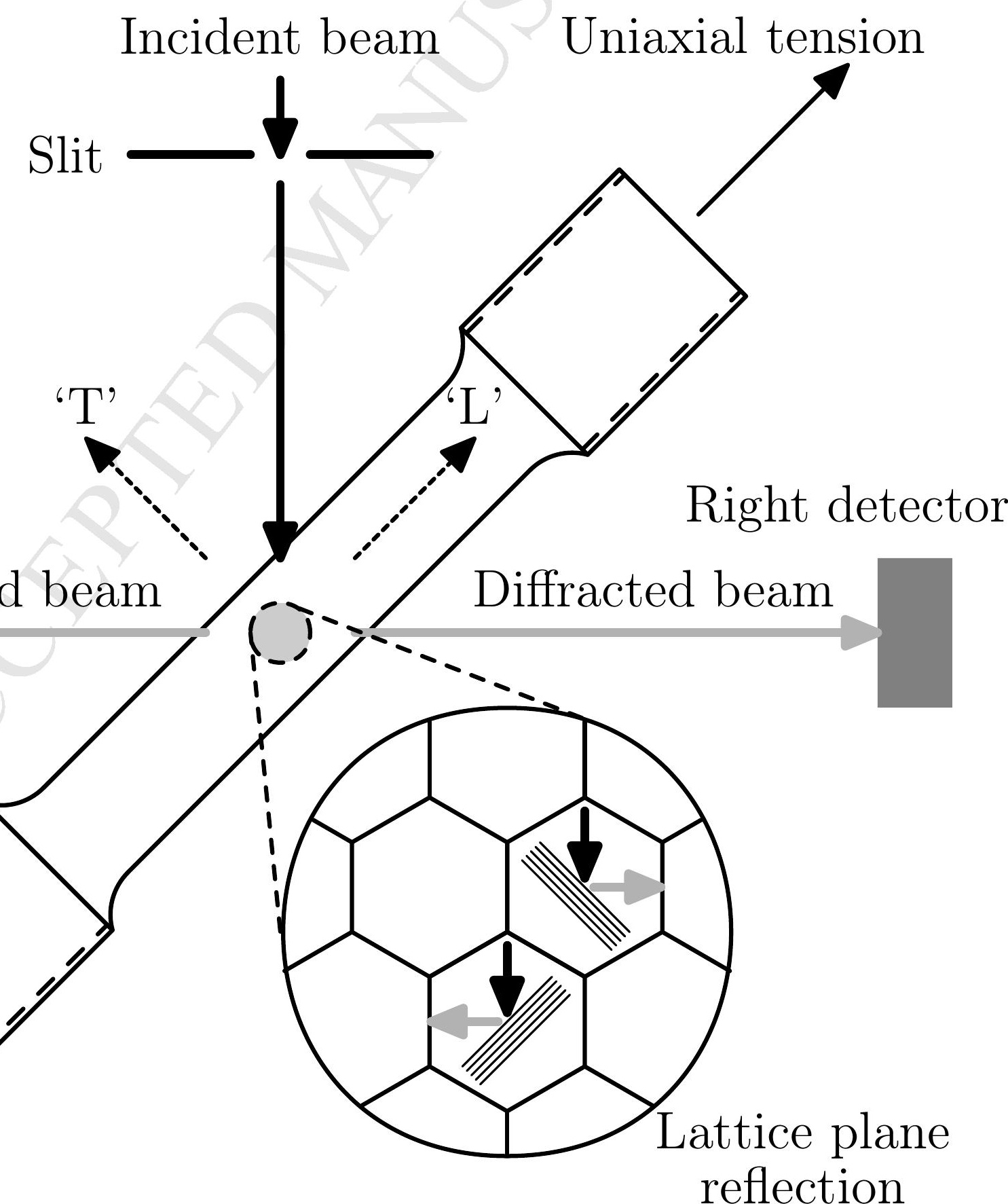

\section{Diffracted beam} reflection 


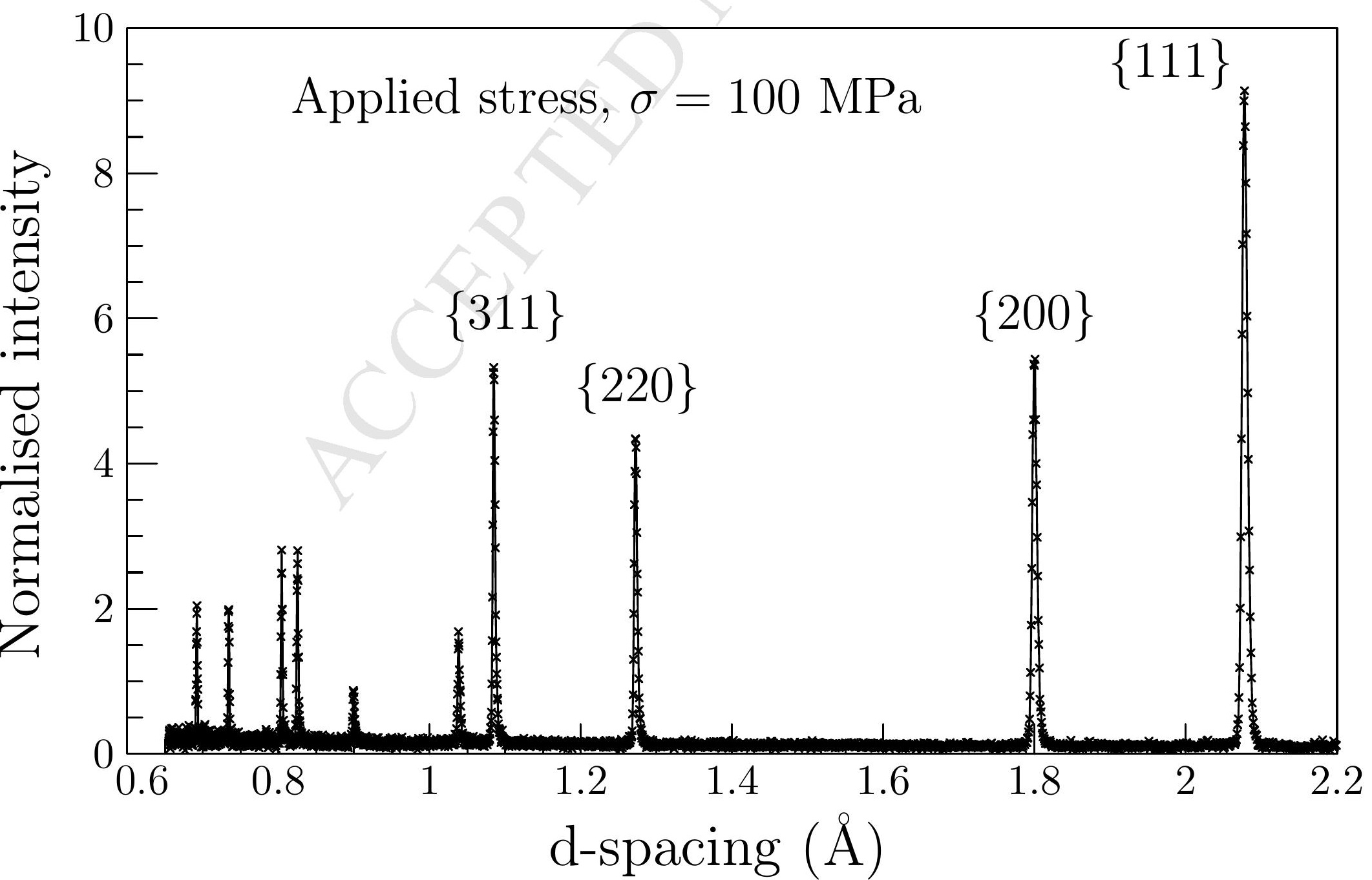


$x_{2}$

\section{G1 G2}

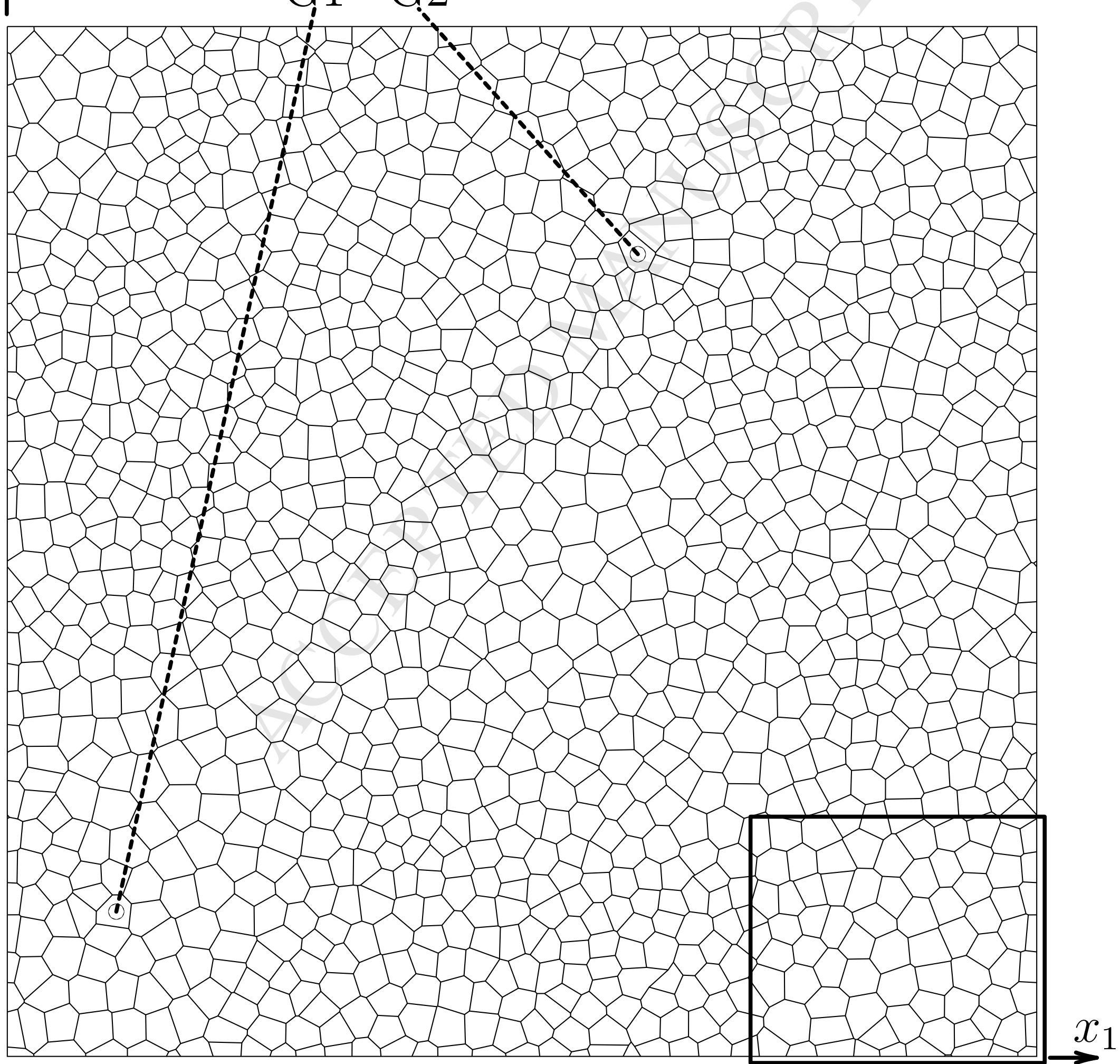




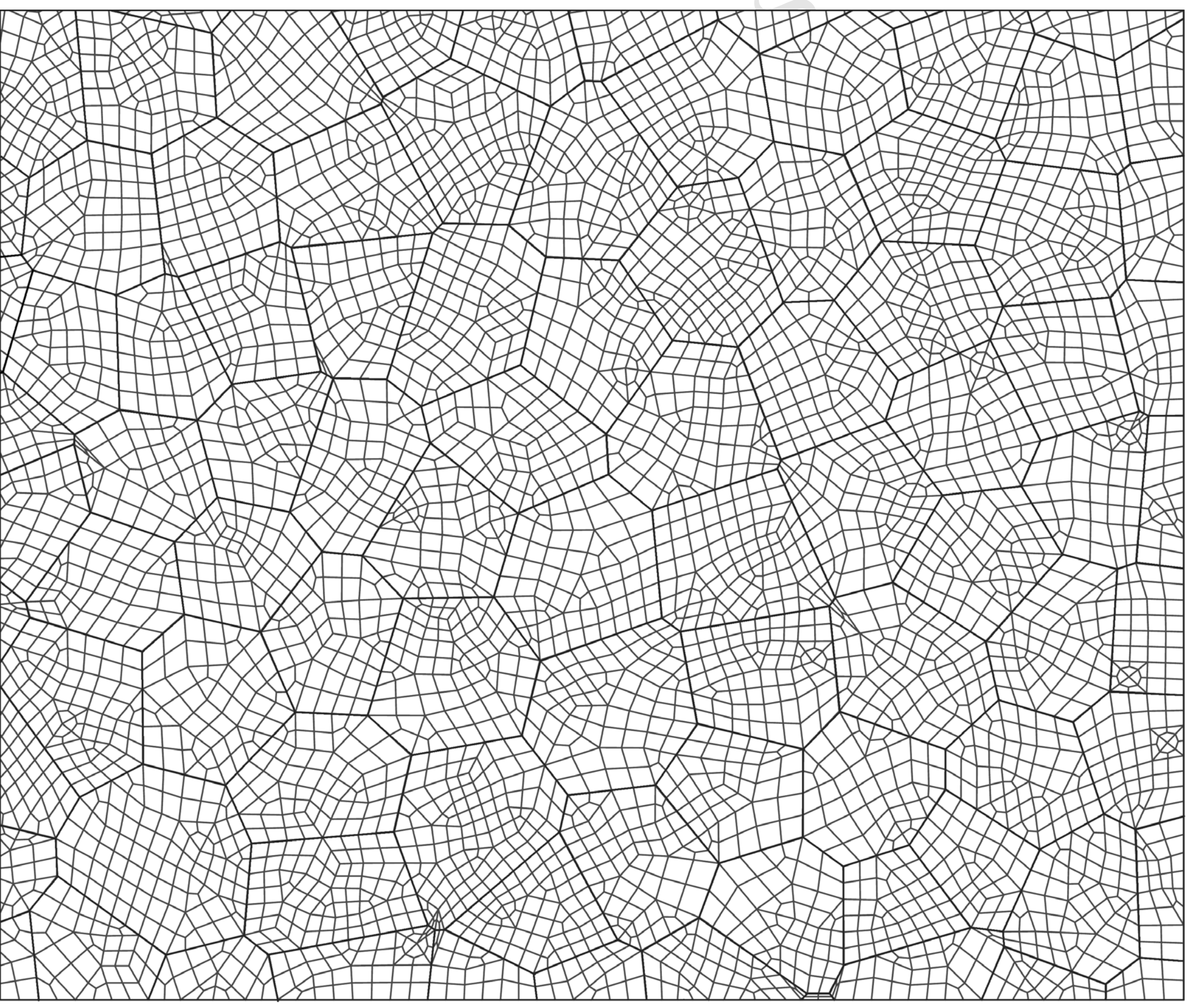




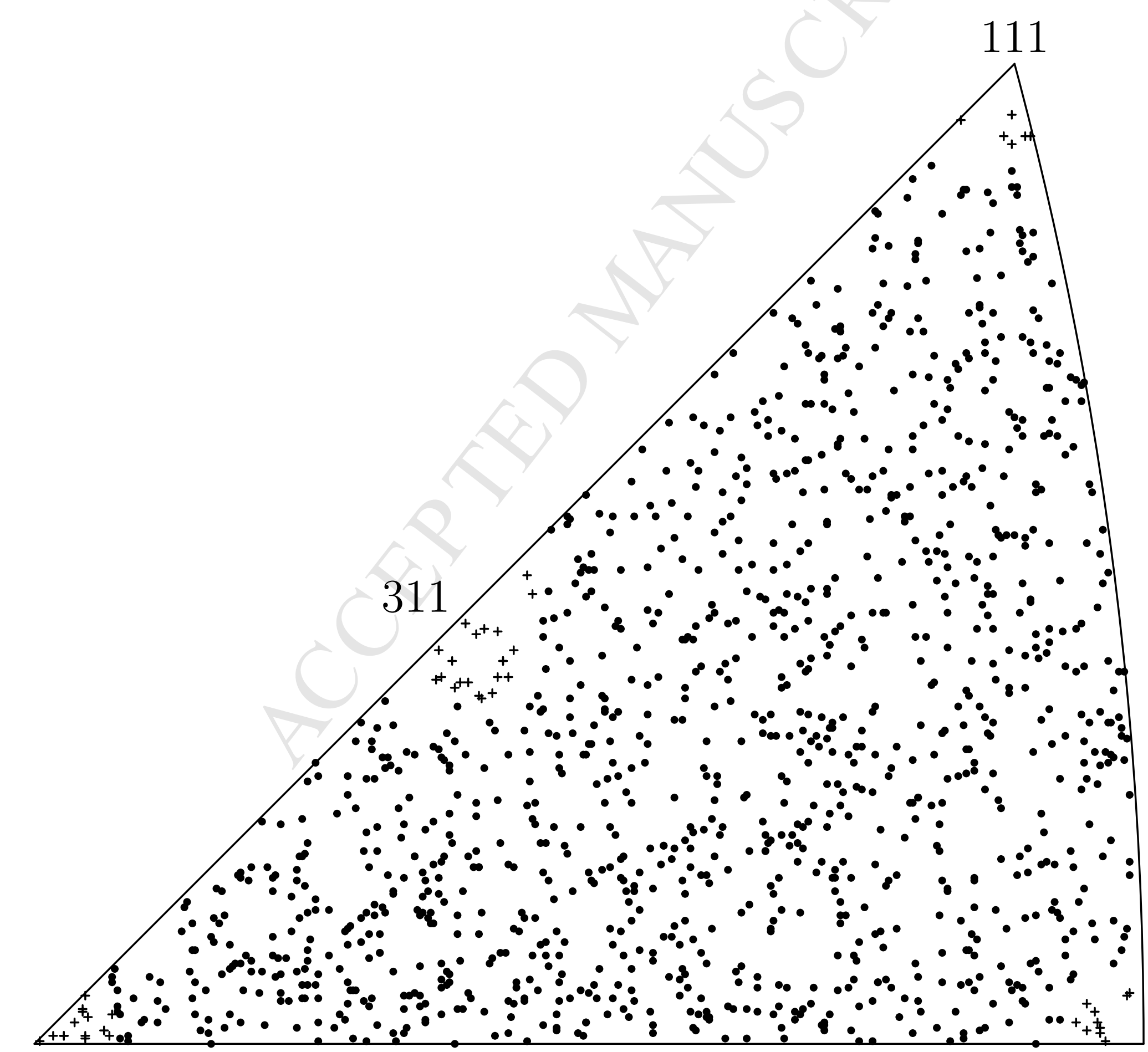




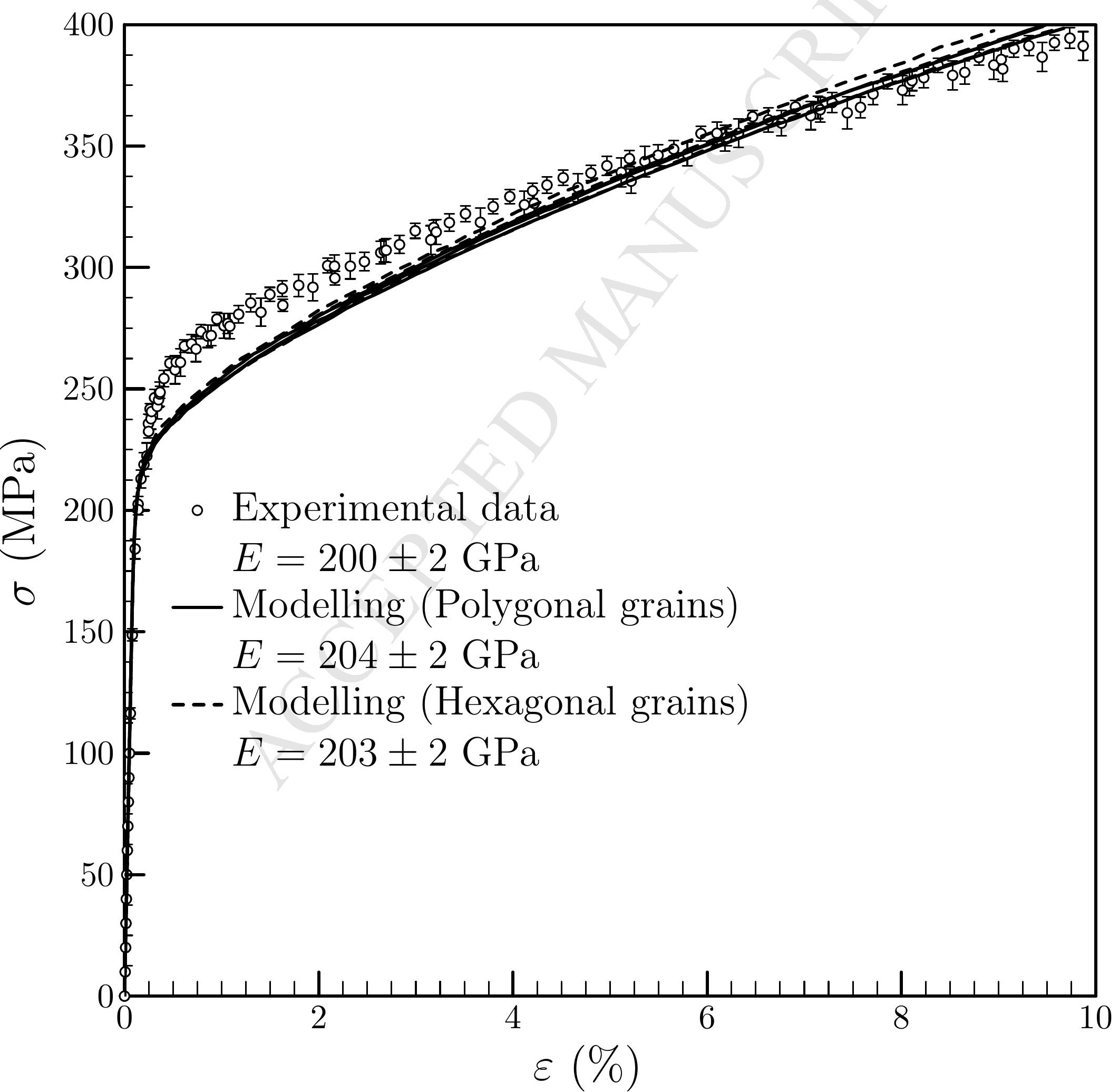




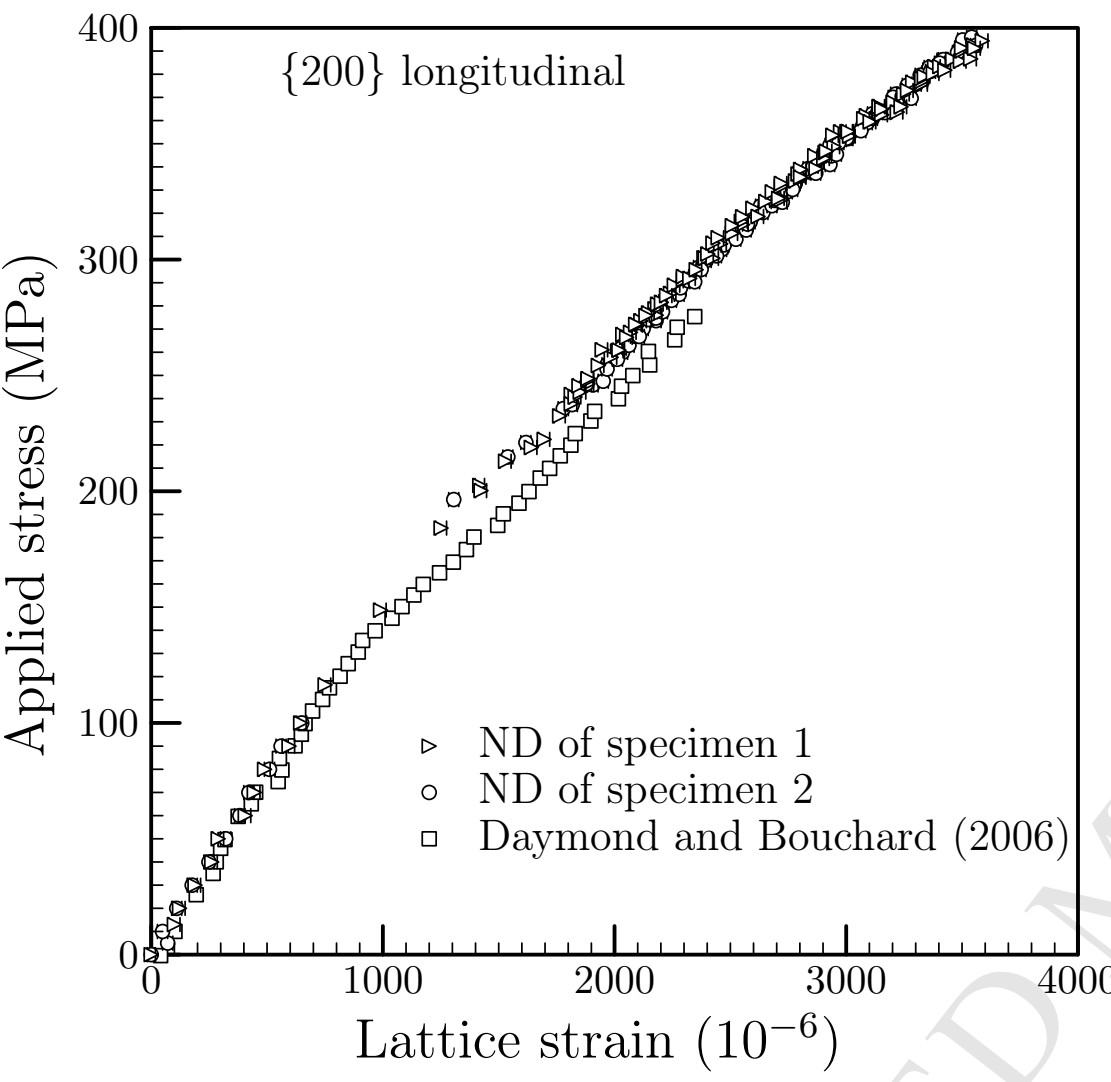

(a)

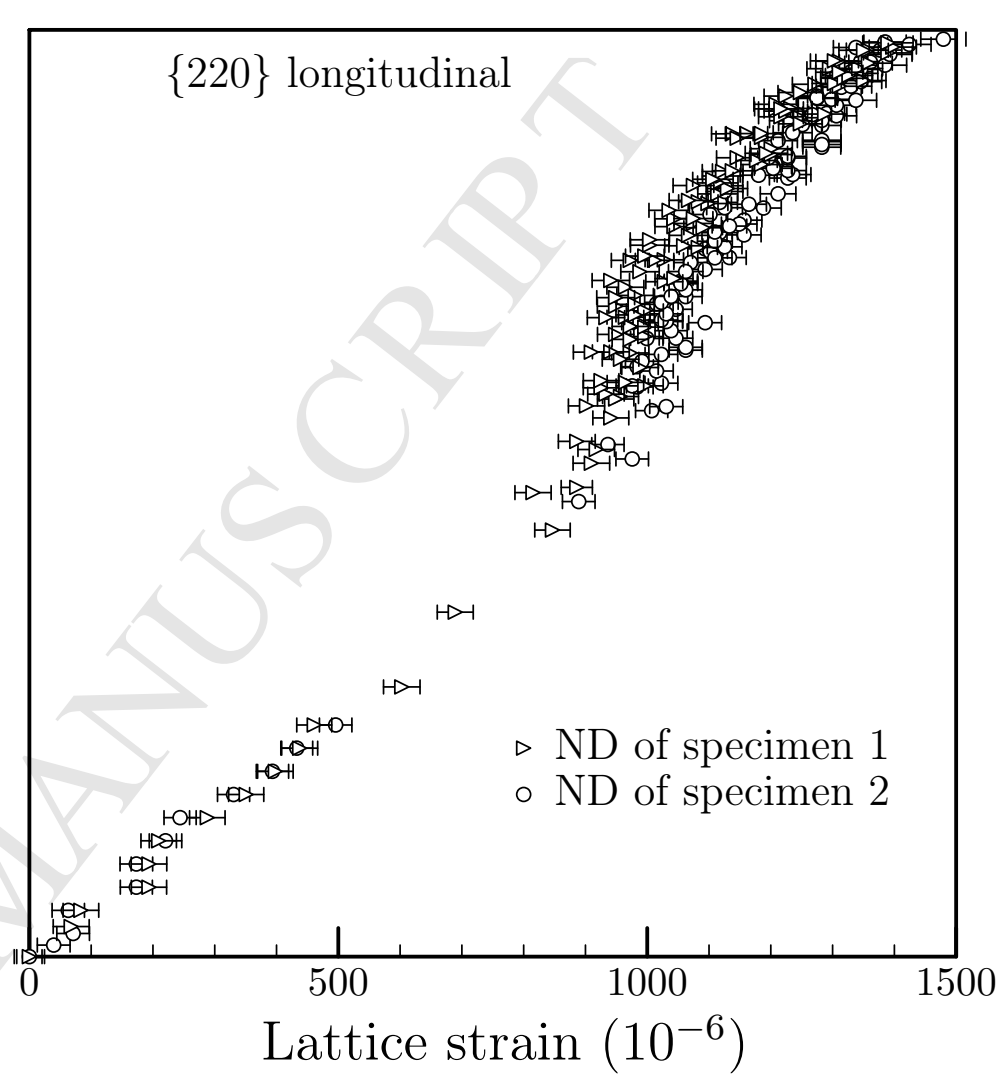

(b)

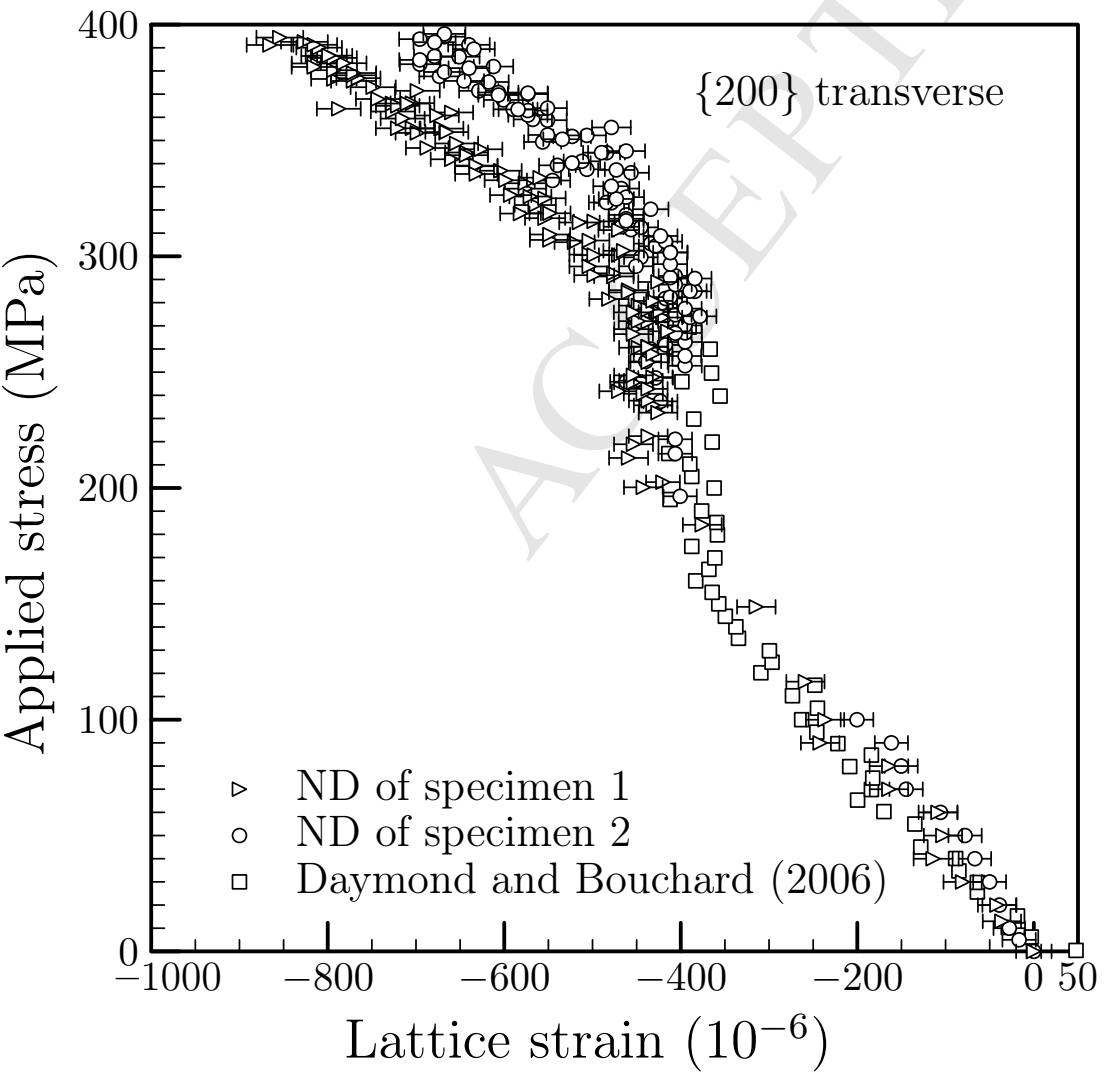

(c)

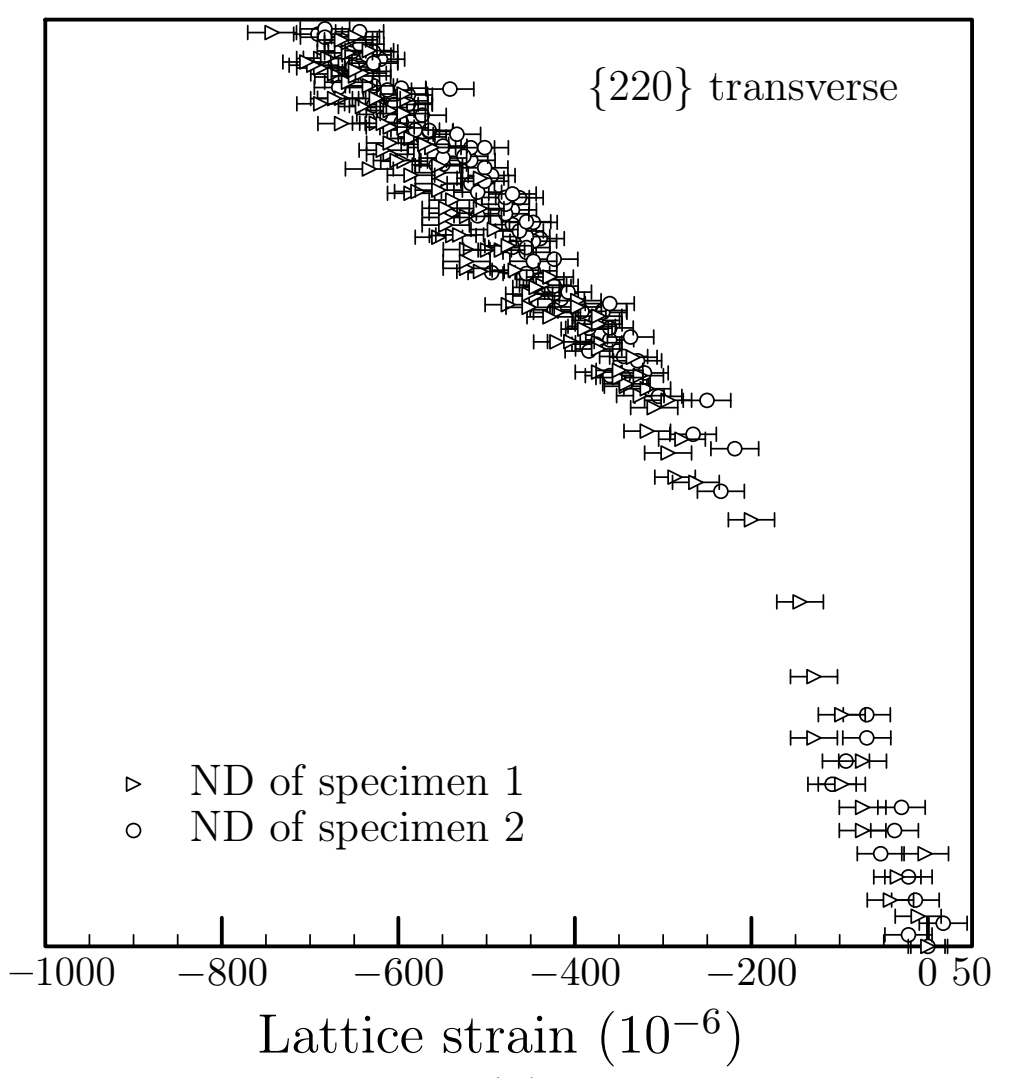

(d) 


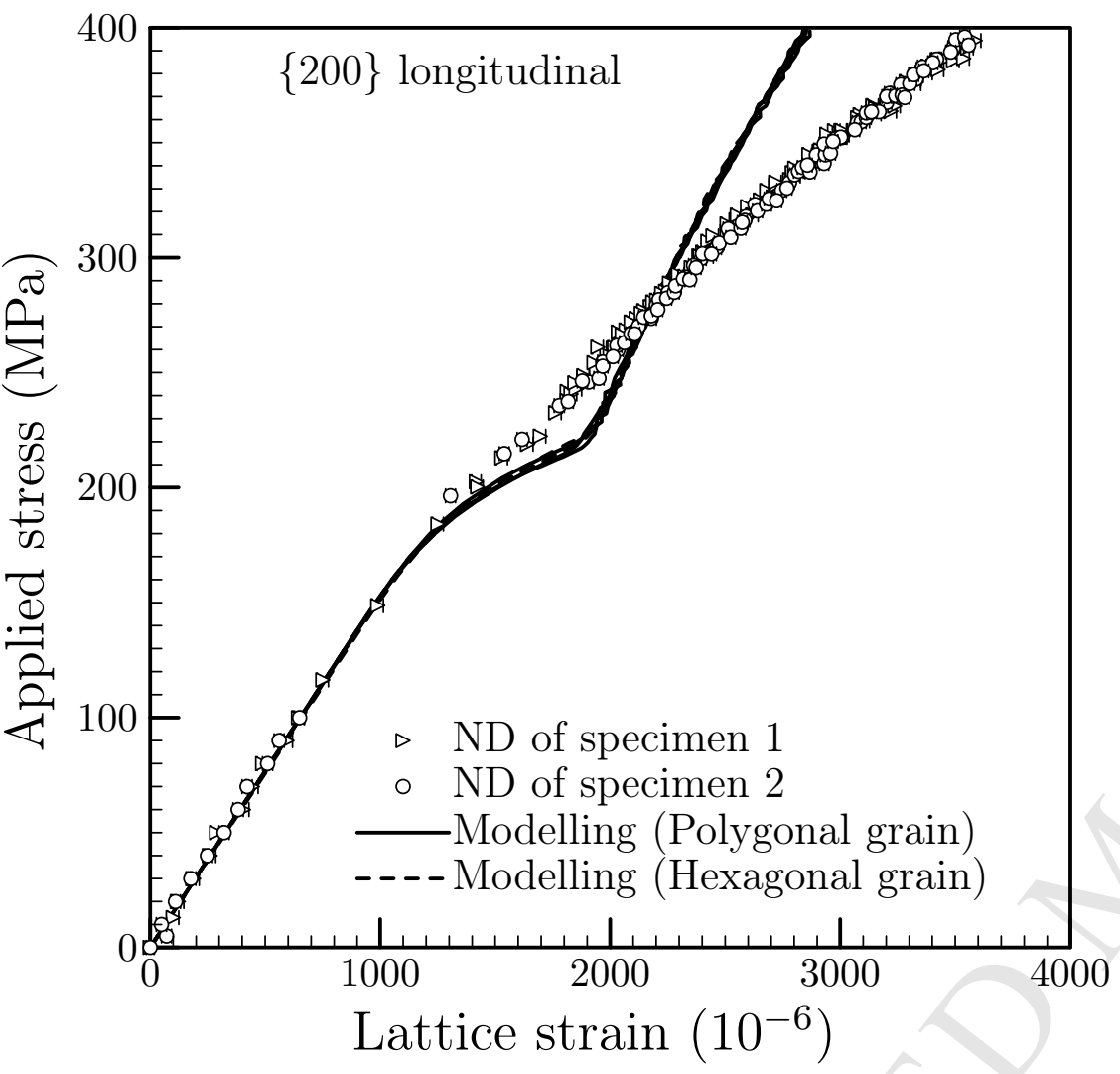

(a)

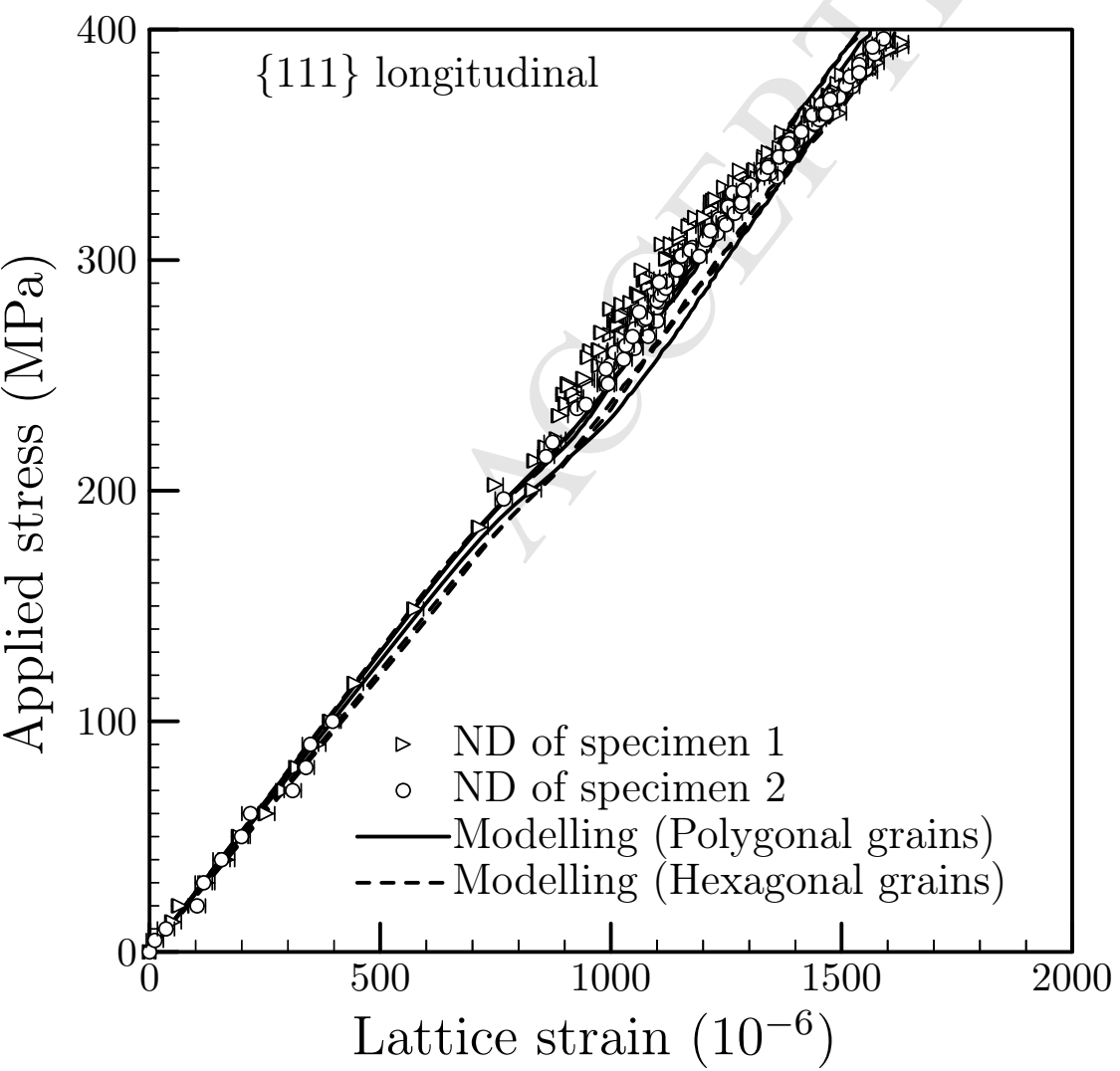

(c)

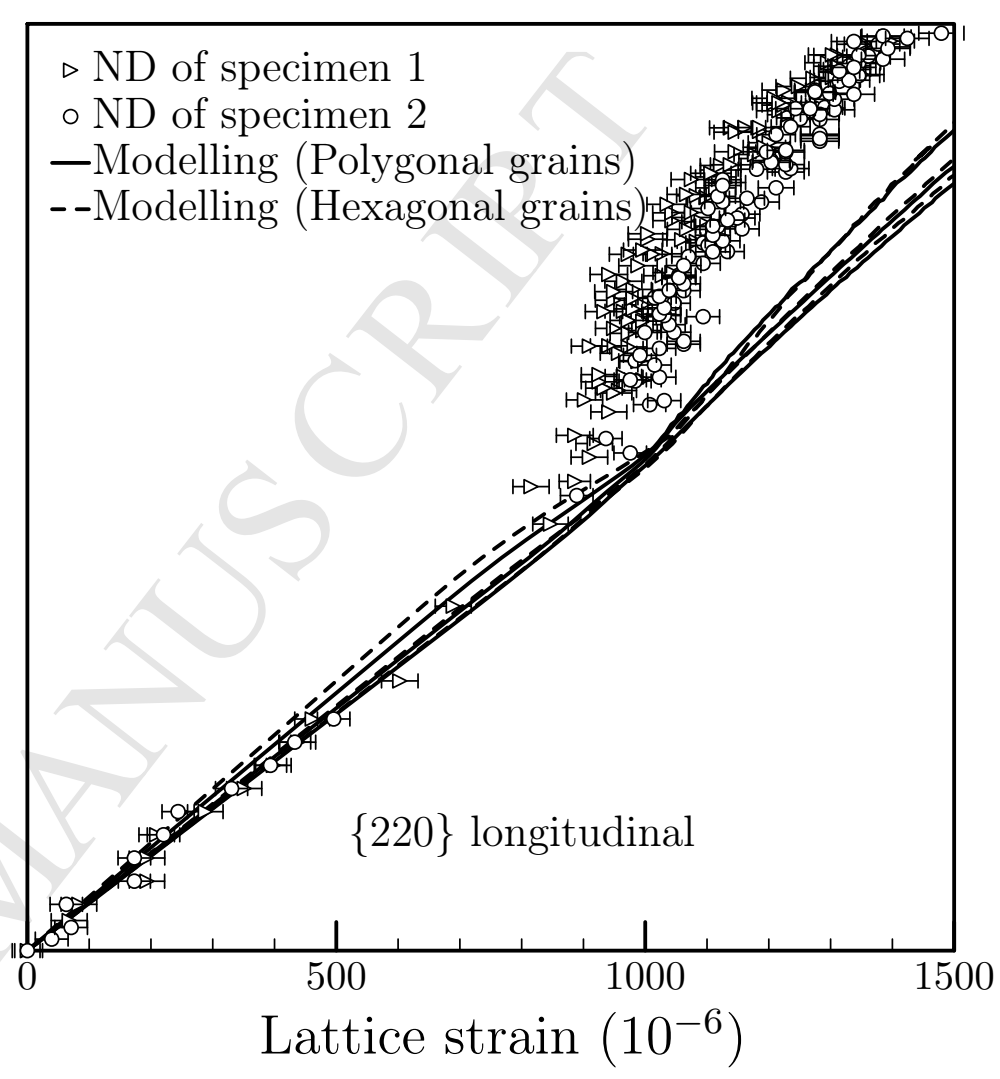

(b)

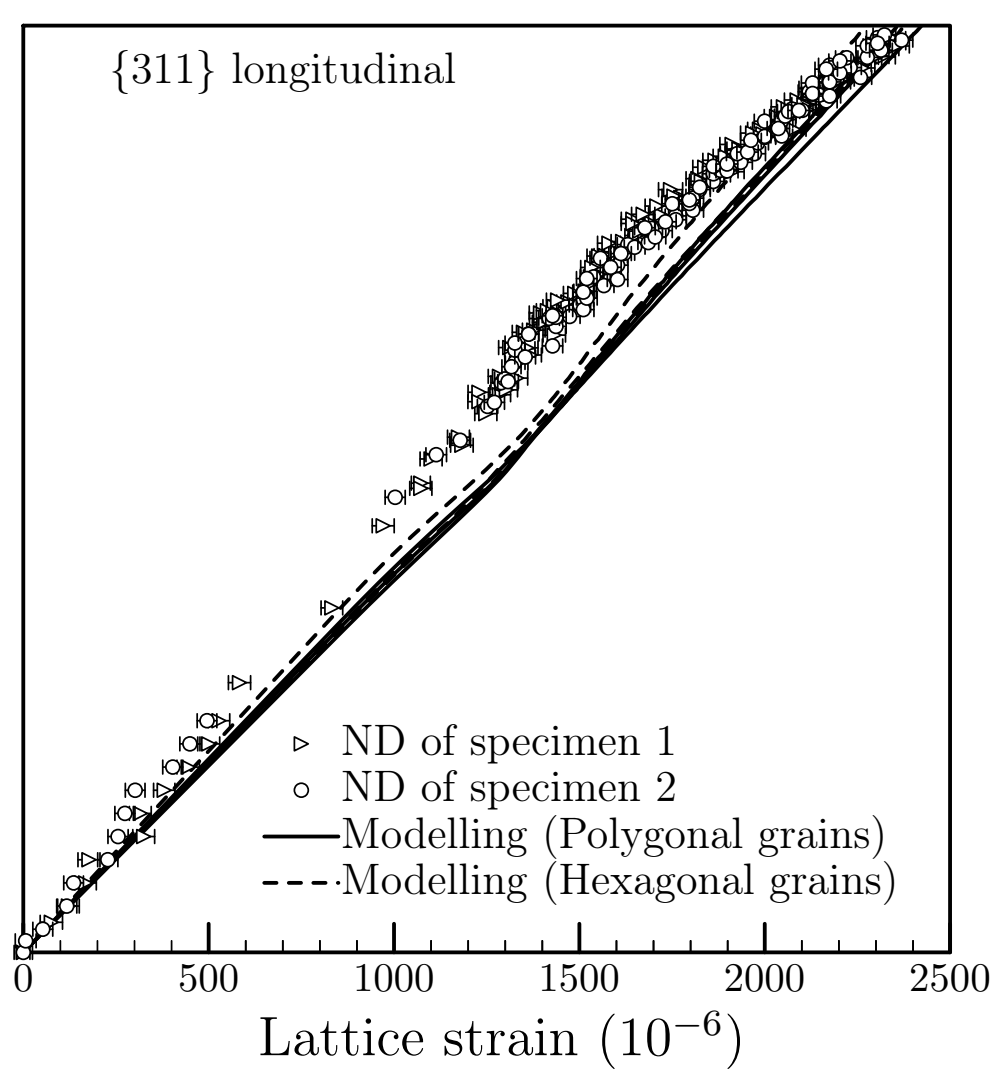

(d) 


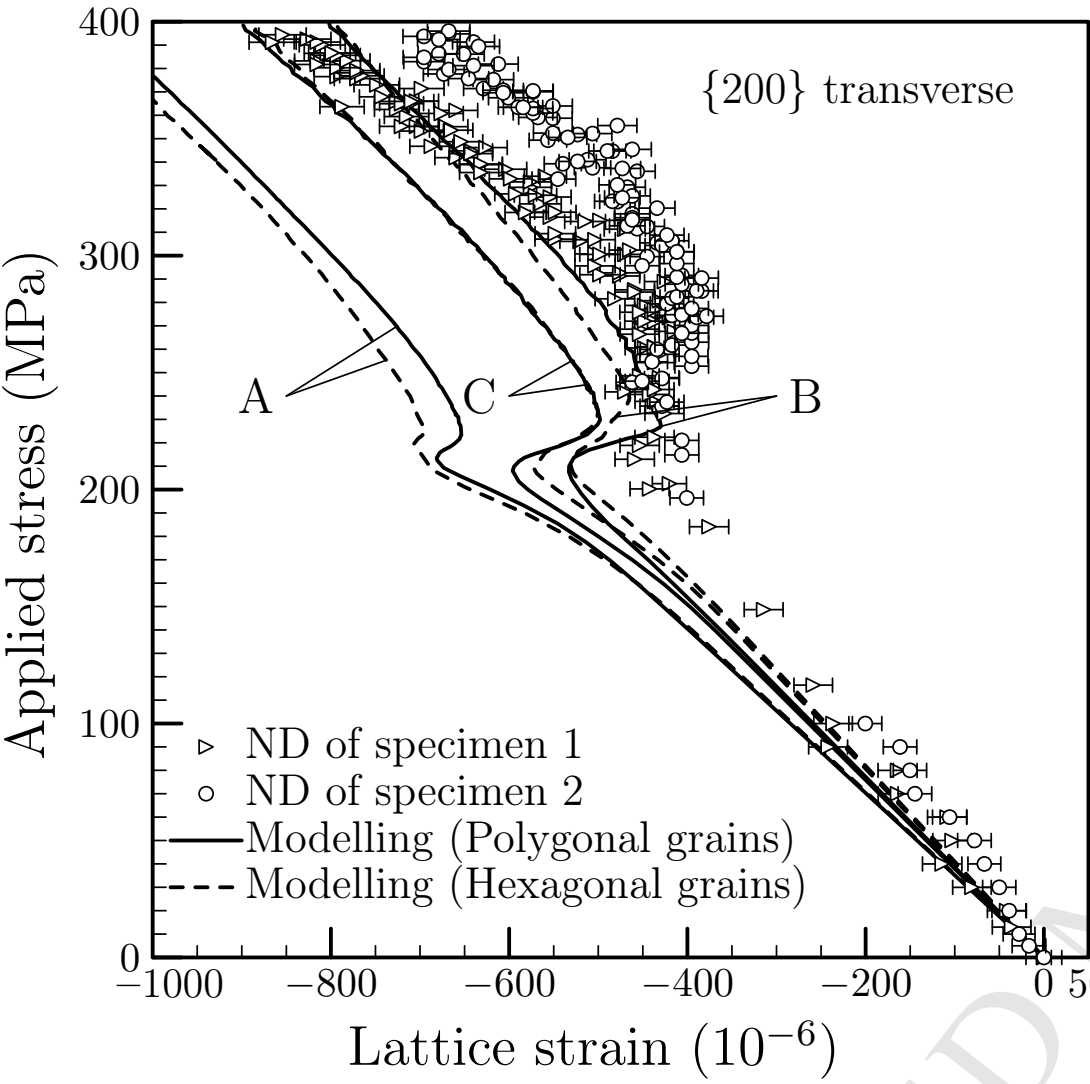

(a)

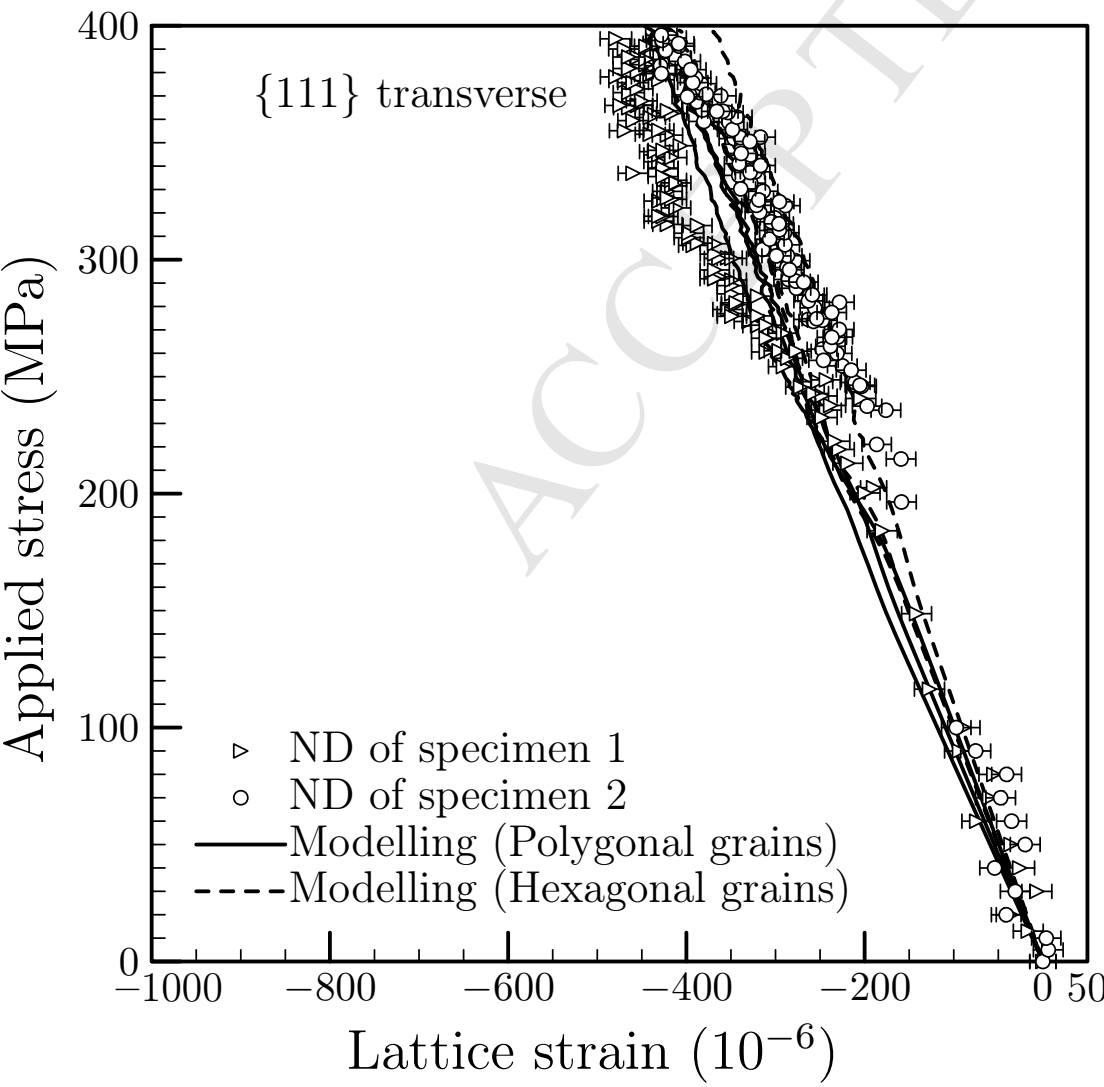

(c)

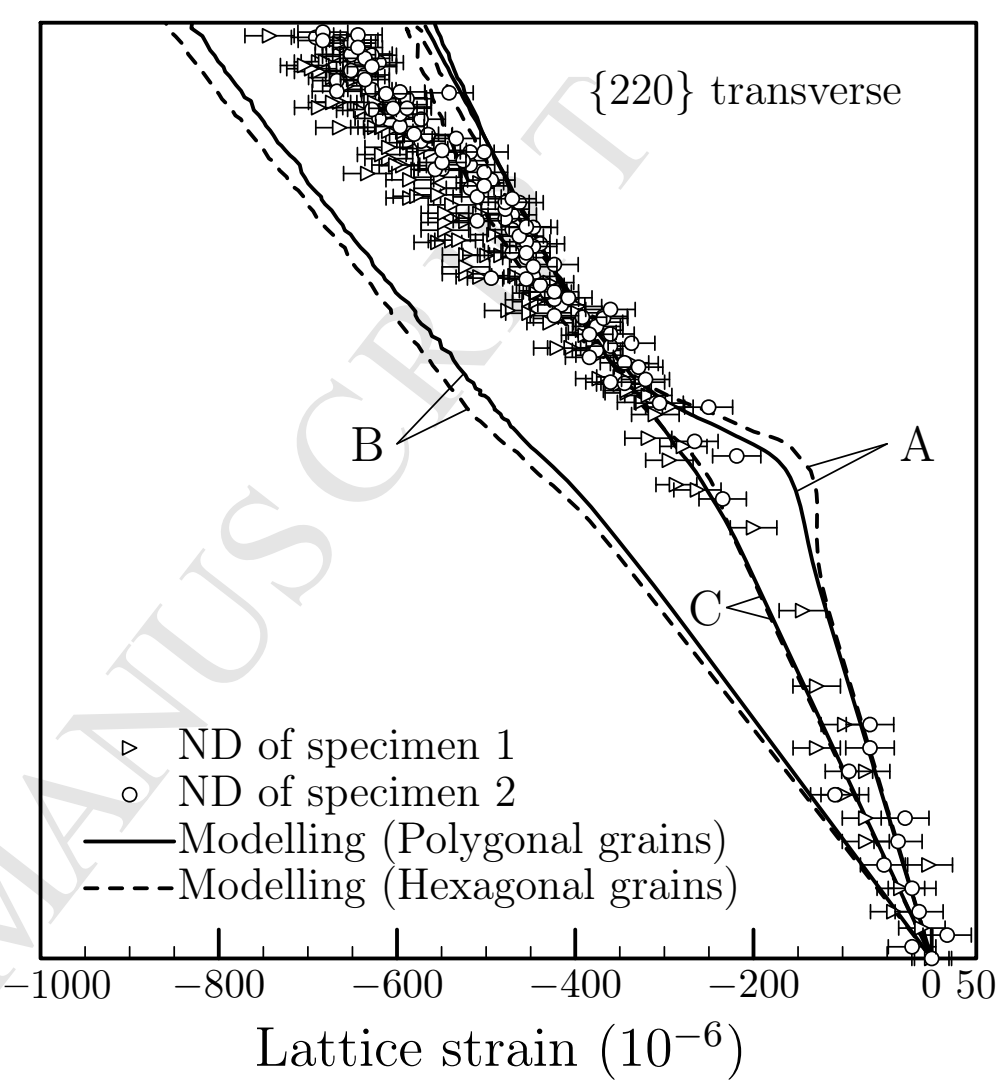

(b)

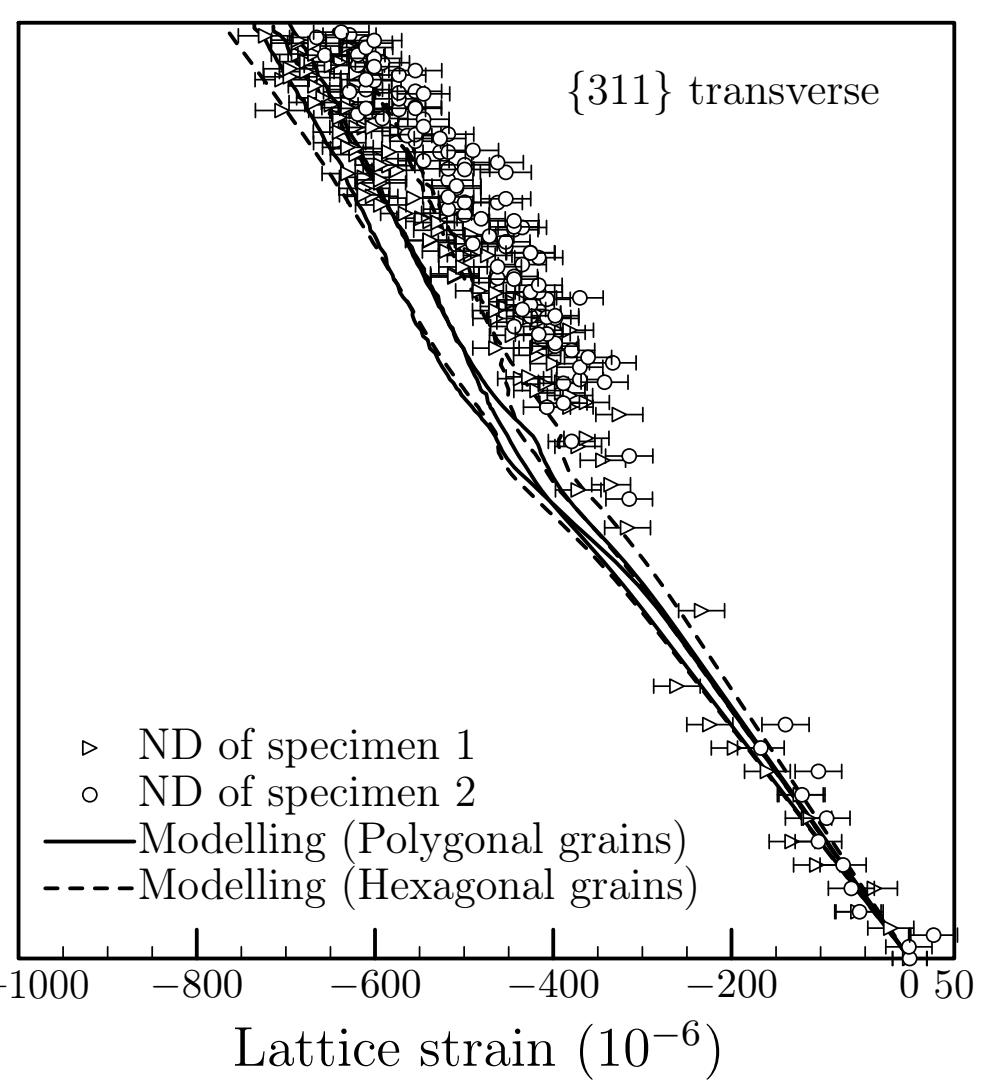

(d) 


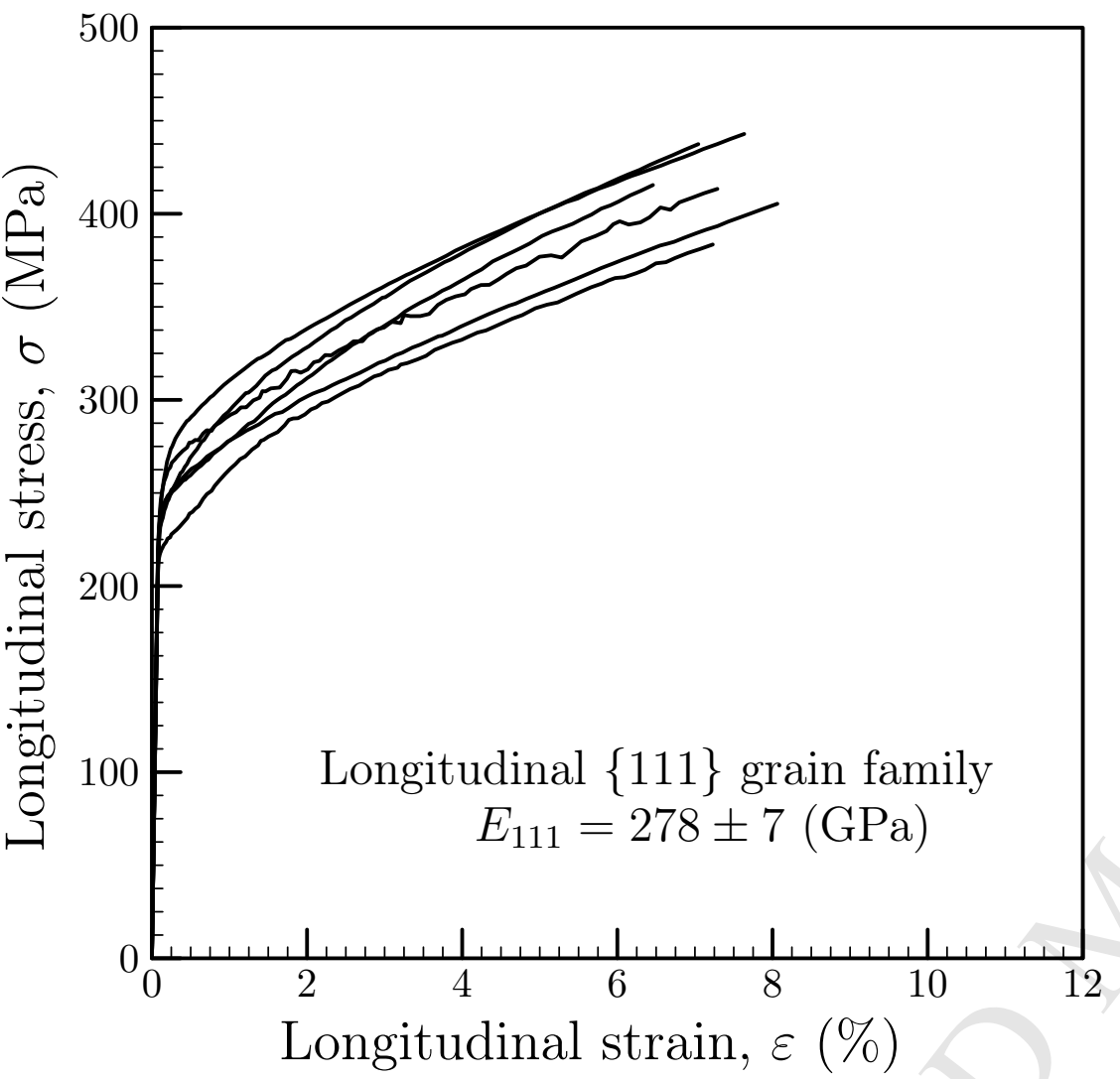

(a)

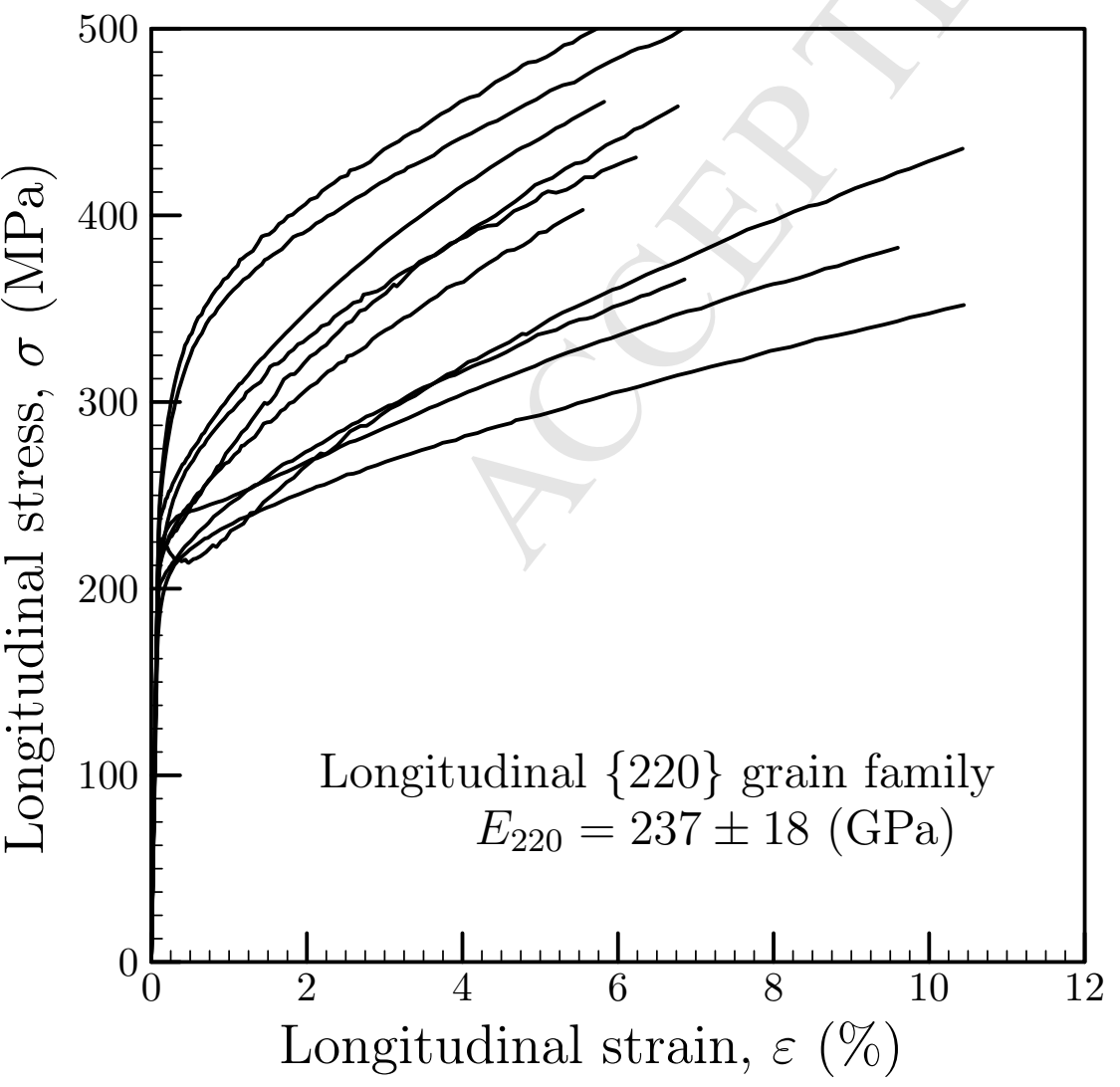

(c)

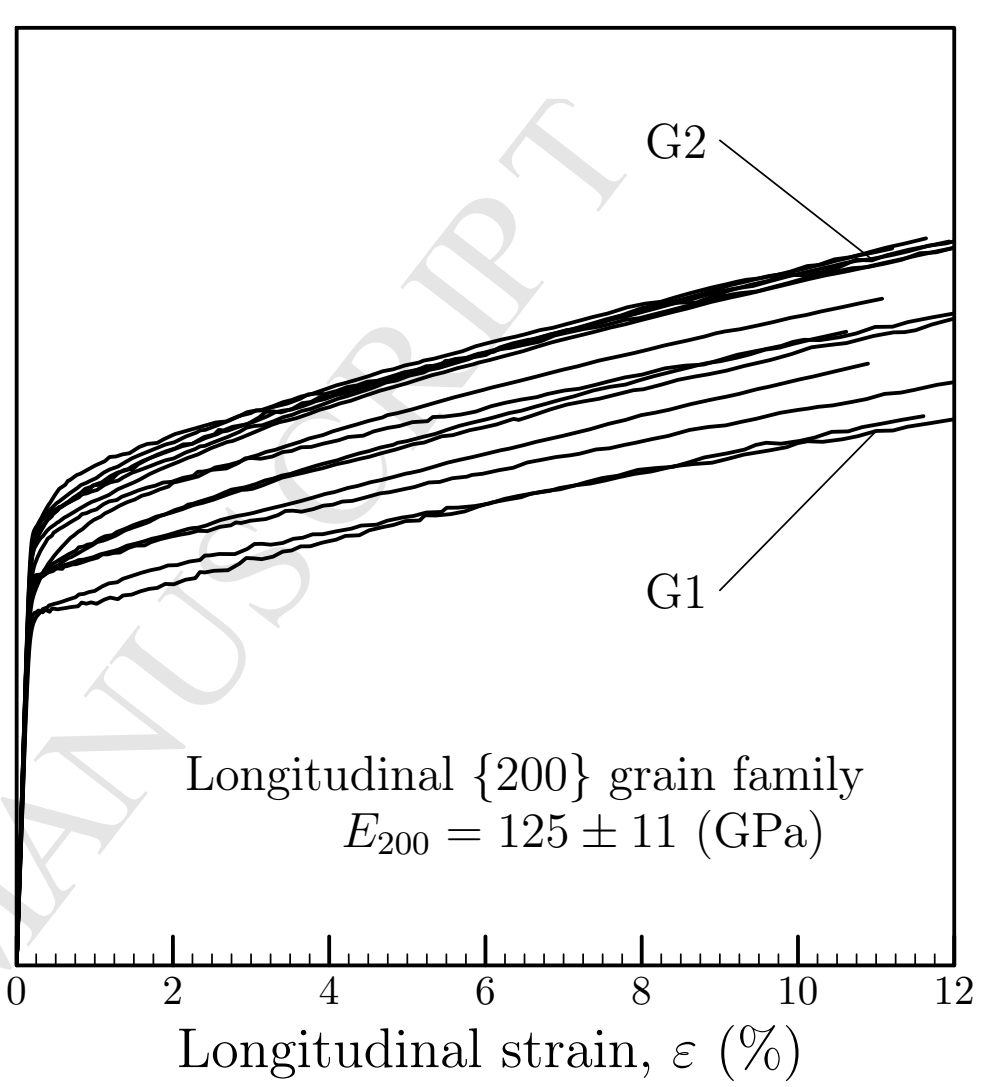

(b)

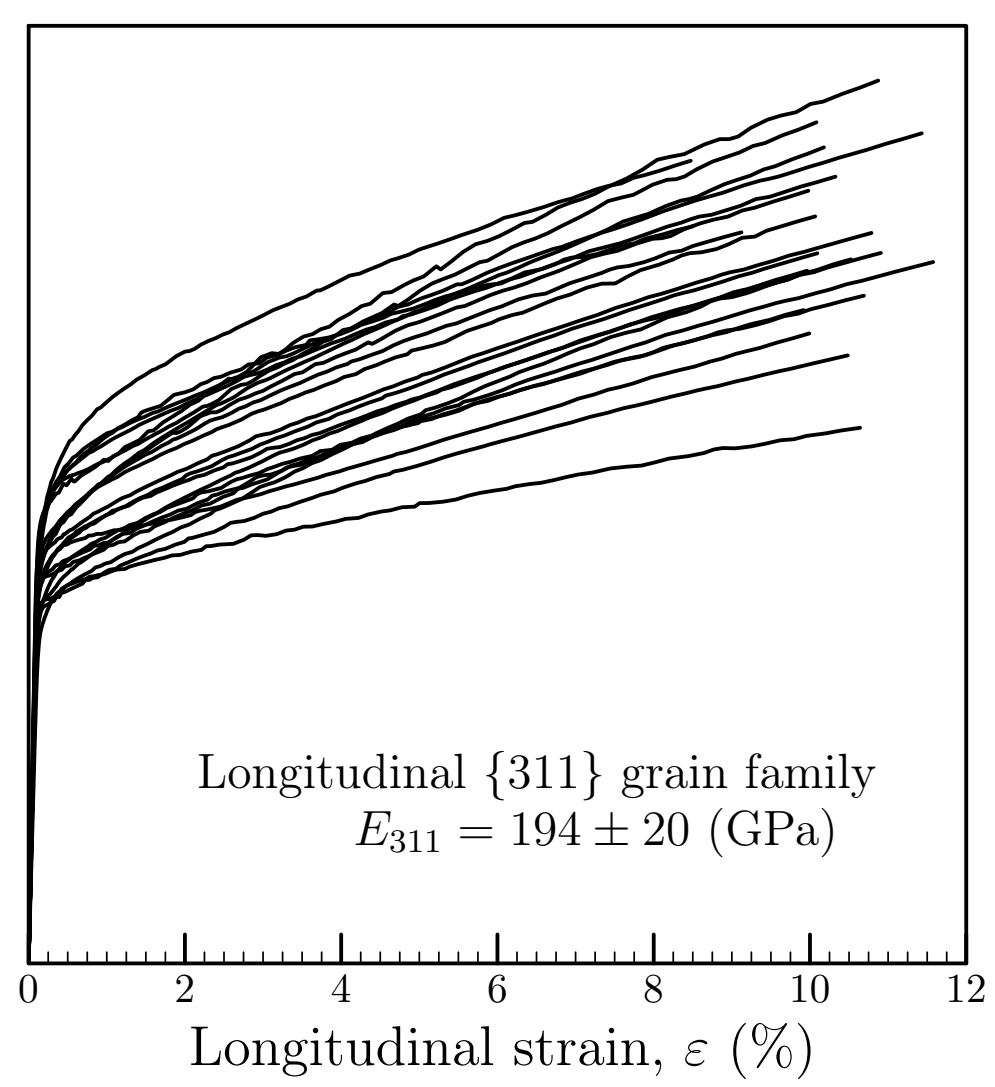

(d) 

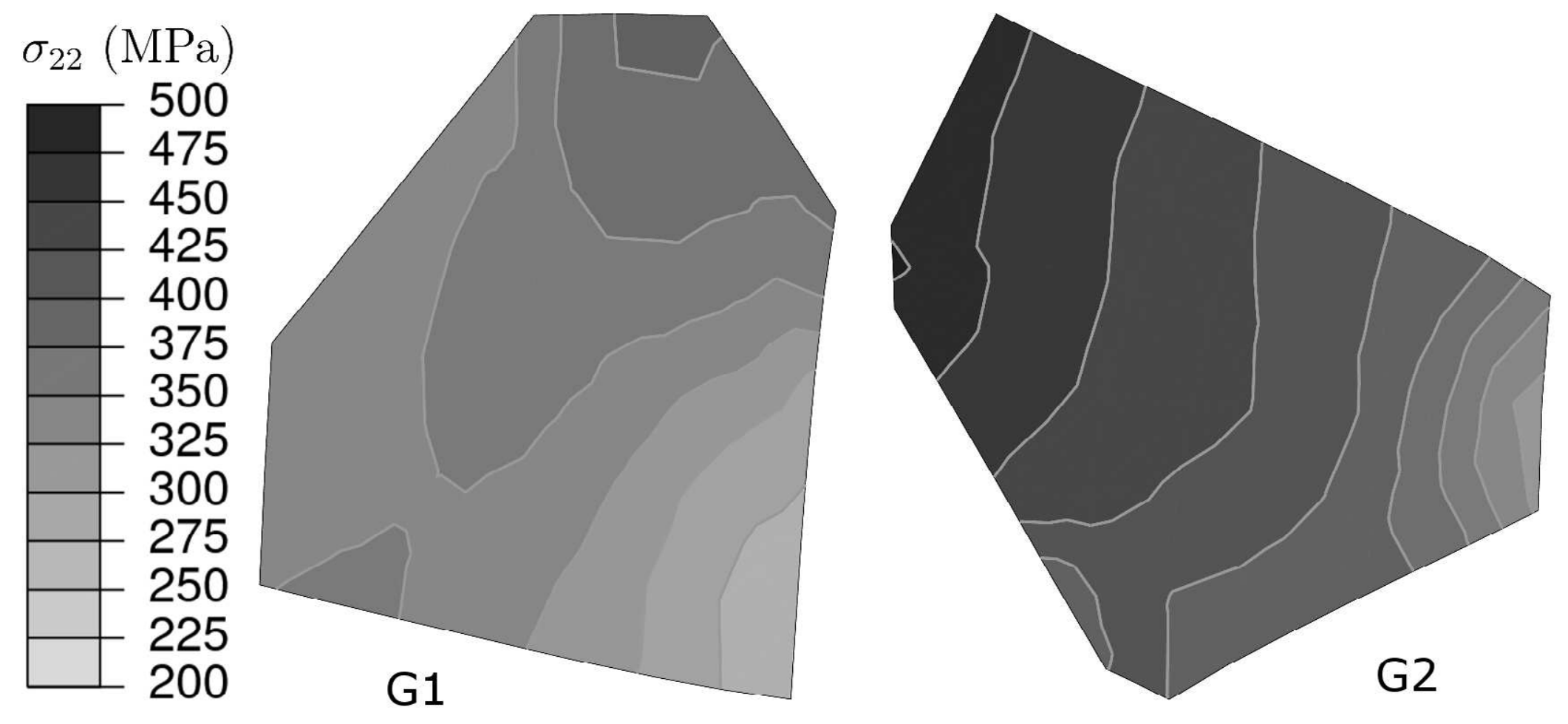


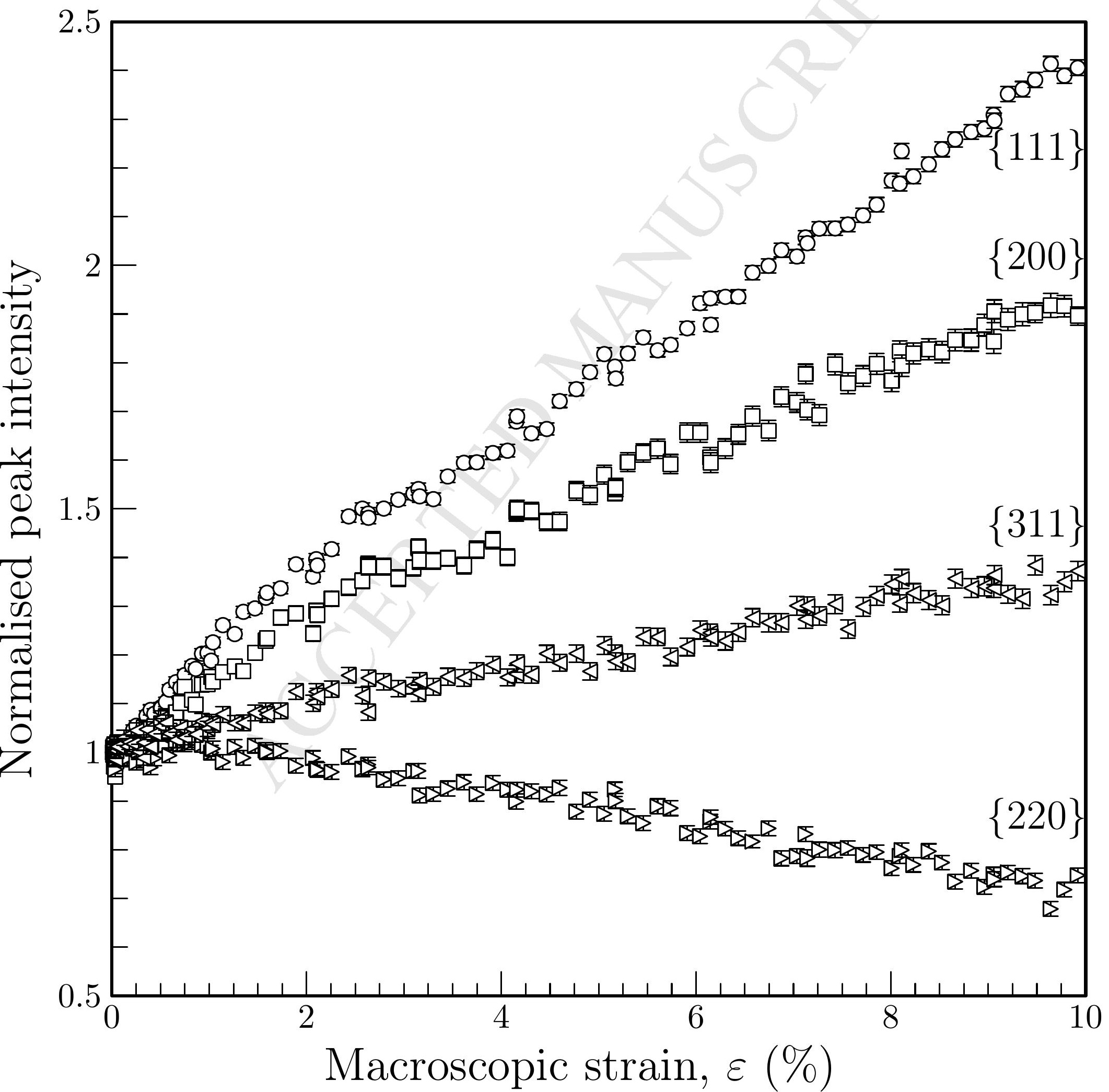




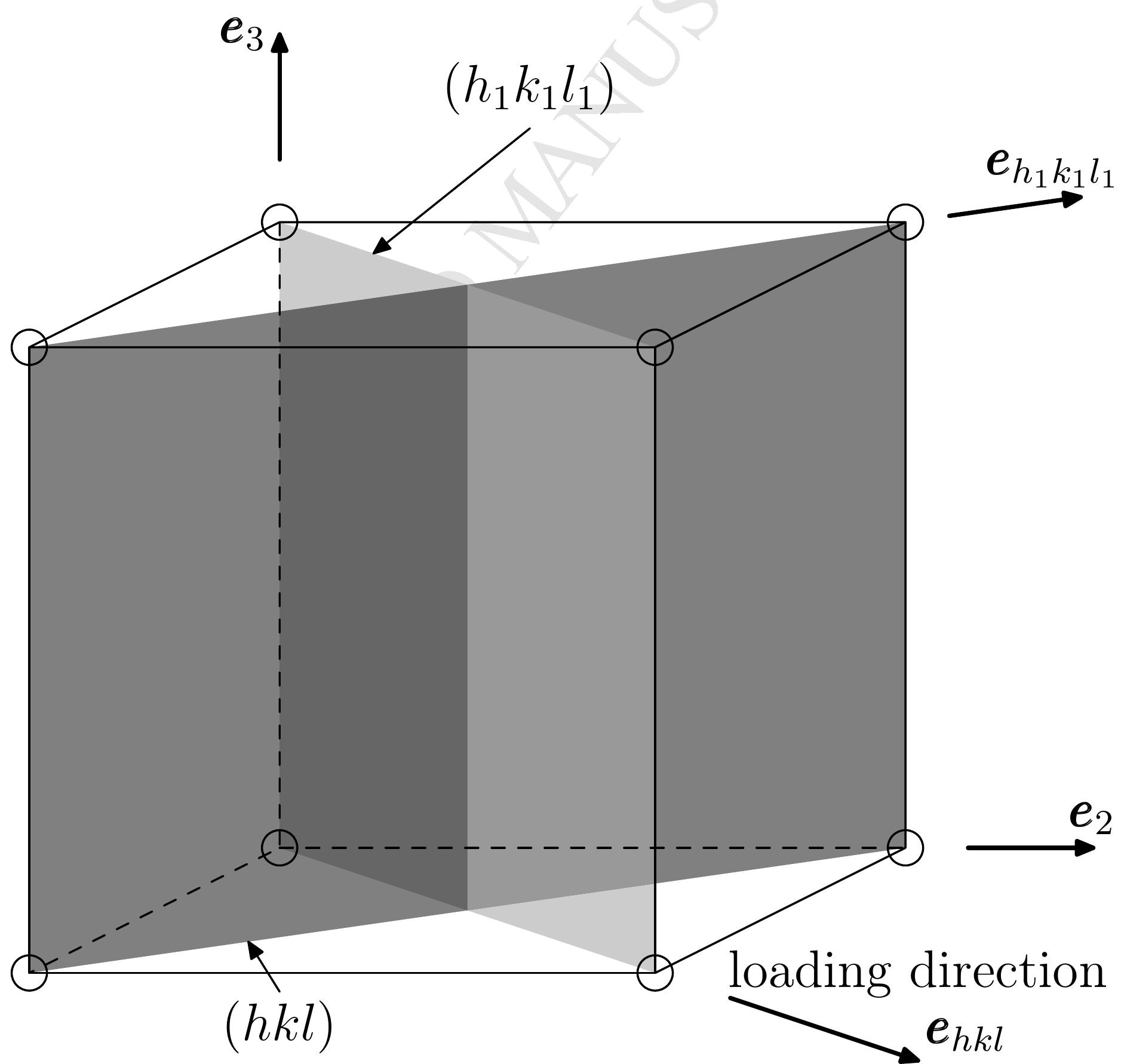

